\title{
CONSTANT SCALAR CURVATURE METRICS ON CONNECTED SUMS
}

\author{
DOMINIC JOYCE
}

Received 13 August 2001 and in revised form 12 March 2002

\begin{abstract}
The Yamabe problem (proved in 1984) guarantees the existence of a metric of constant scalar curvature in each conformal class of Riemannian metrics on a compact manifold of dimension $n \geq 3$, which minimizes the total scalar curvature on this conformal class. Let $\left(M^{\prime}, g^{\prime}\right)$ and $\left(M^{\prime \prime}, g^{\prime \prime}\right)$ be compact Riemannian $n$-manifolds. We form their connected sum $M^{\prime} \# M^{\prime \prime}$ by removing small balls of radius $\epsilon$ from $M^{\prime}, M^{\prime \prime}$ and gluing together the $\mathscr{\varphi}^{n-1}$ boundaries, and make a metric $g$ on $M^{\prime} \# M^{\prime \prime}$ by joining together $g^{\prime}, g^{\prime \prime}$ with a partition of unity. In this paper, we use analysis to study metrics with constant scalar curvature on $M^{\prime} \# M^{\prime \prime}$ in the conformal class of $g$. By the Yamabe problem, we may rescale $g^{\prime}$ and $g^{\prime \prime}$ to have constant scalar curvature 1,0 , or -1 . Thus, there are nine cases, which we handle separately. We show that the constant scalar curvature metrics either develop small "necks" separating $M^{\prime}$ and $M^{\prime \prime}$, or one of $M^{\prime}, M^{\prime \prime}$ is crushed small by the conformal factor. When both sides have positive scalar curvature, we find three metrics with scalar curvature 1 in the same conformal class.
\end{abstract}

2000 Mathematics Subject Classification: 58E11, 53C20.

1. Introduction. Let $\left(M^{\prime}, g^{\prime}\right)$ and $\left(M^{\prime \prime}, g^{\prime \prime}\right)$ be compact manifolds of dimension $n \geq 3$. The connected sum $M=M^{\prime} \# M^{\prime \prime}$ is the result of removing a small ball $B^{n}$ from each manifold, and joining the resulting manifolds at their common boundary $\mathscr{S}^{n-1}$. By smoothly joining $g^{\prime}$ and $g^{\prime \prime}$, we can also construct a 1-parameter family of metrics $g_{t}$ on $M$ for $t \in(0, \delta)$, where $t$ measures the radius of the excised balls $B^{n}$.

In this paper, we suppose $g^{\prime}$ and $g^{\prime \prime}$ have constant scalar curvature (not necessarily the same value), and study (some of) the metrics $\tilde{g}_{t}$ with constant scalar curvature in the conformal class of $g_{t}$ for small $t$. Our method is to write down explicit metrics whose scalar curvature is close to constant and show, using analysis, that they can be adjusted by a small conformal change to give metrics with constant scalar curvature.

By the proof of the Yamabe problem, every conformal class on $M$ contains a metric with constant scalar curvature. Our proofs are simpler than the solution of the Yamabe problem, as our problem is much easier, and they have the advantage of giving a good grasp of what the Yamabe metrics actually look like as the underlying conformal manifold decays into a connected sum. We can, for instance, say that one obvious sort of behaviour, that of developing 
long "tubes" resembling $\mathscr{9}^{n-1} \times \mathbb{R}$, does not happen, but that small "pinched necks" may develop instead, or else $M^{\prime}$ or $M^{\prime \prime}$ may be crushed very small by the conformal factor.

Other authors have also considered the scalar curvature of metrics on connected sums. Two important early papers on manifolds with positive scalar curvature are Gromov and Lawson [3] and Schoen and Yau [11], and both show that the connected sum of two manifolds with positive scalar curvature carries metrics with positive scalar curvature (see [3, Theorem A] and [11, Corollary 3]).

Writing after the solution of the Yamabe problem, Kobayashi [6] defines the Yamabe number of a manifold to be the supremum over conformal classes of the Yamabe invariant defined in Section 1.2 of this paper, and proves an inequality [6, Theorem 2(a)] between the Yamabe number of two manifolds and the Yamabe number of their connected sum. His formula can be related to our results below in the limit $t \rightarrow 0$.

The first version of this work formed part of the author's Ph.D. thesis [5] in 1992, supervised by Simon Donaldson. In the interval between then and the publication of the present paper, several papers on similar topics have appeared, and we mention in particular Mazzeo et al. [8]. They study connected sums of compact or noncompact manifolds with constant positive scalar curvature, and the part of Theorem 3.9, dealing with the metrics of Section 2.2, also follows from their main result.

The remainder of this section goes over some necessary background material. In Section 2, we define two families of metrics $g_{t}$ upon connected sums, make estimates of the scalar curvature of these metrics, and prove a uniform bound on a Sobolev constant for a particular embedding of Sobolev spaces.

The main existence results for metrics of constant scalar curvature conformal to the metrics $g_{t}$ above are proved in Section 3. We begin with a quite general existence proof using a sequence method and then apply it, first to the case of scalar curvature -1 and then to the case of scalar curvature 1 . The former is simple, but the latter is more difficult, and the proof of a result on the eigenvalues of the Laplacian $\Delta$ on the metrics $g_{t}$ in the positive case has been deferred until the appendix.

In Section 4, we deal with the cases left over from Section 3 which are the connected sums involving manifolds of zero scalar curvature. To do so, this requires some additions to the methods of Section 3, as the problem of rescaling a metric with scalar curvature close to \pm 1 to get exactly \pm 1 is different from the problem of rescaling a metric with scalar curvature close to zero to get scalar curvature of a small but unknown constant. We will see that each combination of positive, negative, and zero scalar curvature manifolds has distinctive features.

1.1. Analytic preliminaries. We define Lebesgue and Sobolev spaces, principally to establish notation. An introduction to them may be found in Aubin 
[1, Section 2]. Let $(M, g)$ be a Riemannian manifold. For $q \geq 1$, the Lebesgue space $L^{q}(M)$ is the set of locally integrable functions $u$ on $M$ for which the norm

$$
{ }^{g}\|u\|_{L^{q}}=\|u\|_{L^{q}}=\left(\int_{M}|u|^{q} d V_{g}\right)^{1 / q}
$$

is finite. Here $d V_{g}$ is the volume form of the metric $g$. Then $L^{q}(M)$ is a Banach space (with the convention that two functions are equal if they differ only on a null set) and $L^{q}(M)$ a Hilbert space. Let $r, s, t \geq 1$ with $1 / r=1 / s+1 / t$. If $\phi \in L^{s}(M)$ and $\psi \in L^{t}(M)$, then $\phi \psi \in L^{r}(M)$ and $\|\phi \psi\|_{L^{r}} \leq\|\phi\|_{L^{s}}\|\psi\|_{L^{t}}$; this is Hölder inequality.

Let $q \geq 1$ and let $k$ be a nonnegative integer. The Sobolev space $L_{k}^{q}(M)$ is the subspace of $u \in L^{q}(M)$ such that $u$ is $k$ times weakly differentiable and $\left|\nabla^{i} u\right| \in L^{q}(M)$ for $i \leq k$. The Sobolev norm on $L_{k}^{q}(M)$ is

$$
{ }^{g}\|u\|_{L_{k}^{q}}=\|u\|_{L_{k}^{q}}=\left(\sum_{i=0}^{k} \int_{M}\left|\nabla^{i} u\right|^{q} d V_{g}\right)^{1 / q} .
$$

This makes $L_{k}^{q}(M)$ a Banach space and $L_{k}^{2}(M)$ a Hilbert space. Since we will often have to consider different metrics on the same manifold, we will use the superscript notation ${ }^{g}\|\cdot\|_{L^{q}}$ and ${ }^{g}\|\cdot\|_{L_{k}^{q}}$ to mean Lebesgue and Sobolev norms computed using the metric $g$.

We also write $C^{k}(M)$ for the vector space of continuous, bounded functions on $M$ with $k$ continuous, bounded derivatives, and $C^{\infty}(M)=\bigcap_{k \geq 0} C^{k}(M)$. The Sobolev embedding theorem [1, Theorem 2.20] says that if $1 / r \geq 1 / q-k / n$, then $L_{k}^{q}(M)$ is continuously embedded in $L^{r}(M)$ by inclusion, and if $-r / n>$ $1 / q-k / n$, then $L_{k}^{q}(M)$ is continuously embedded in $C^{r}(M)$ by inclusion.

1.2. The Yamabe problem. Now we briefly discuss the Yamabe problem, concerning the existence of metrics of constant scalar curvature in a conformal class on a compact manifold. An introduction to this problem and its solution may be found in the survey paper [7] by Lee and Parker.

Fix a dimension $n \geq 3$. Throughout this paper, we will use the notation

$$
a=\frac{4(n-1)}{n-2}, \quad b=\frac{4}{n-2}, \quad p=\frac{2 n}{n-2} .
$$

Let $M$ be a compact manifold of dimension $n$. Define a functional $Q$ upon the set of Riemannian metrics on $M$ by

$$
Q(g)=\frac{\int_{M} S d V_{g}}{\operatorname{vol}(M)^{2 / p}}
$$

where $S$ is the scalar curvature of $g$. Then $Q$ is known as the total scalar curvature or Hilbert action. 
Let $g, \tilde{g}$ be metrics on $M$. We say that $g, \tilde{g}$ are conformal if $\tilde{g}=\phi g$ for some smooth conformal factor $\phi: M \rightarrow(0, \infty)$. Usually we write $\phi=\psi^{p-2}$ for smooth $\psi: M \rightarrow(0, \infty)$. The conformal class $[g]$ of $g$ is the set of metrics $\tilde{g}$ on $M$ conformal to $g$. Applying the calculus of variations to the restriction of $Q$ to a conformal class, we find that a metric $g$ is a stationary point of $Q$ on $[g]$ if and only if it has constant scalar curvature.

Let the scalar curvatures of $g, \tilde{g}$ be $S, \tilde{S}$, and write $\tilde{g}=\psi^{p-2} g$ for smooth $\psi: M \rightarrow(0, \infty)$. Then

$$
\tilde{S}=\psi^{1-p}(a \Delta \psi+S \psi)
$$

Thus $\tilde{\mathfrak{g}}=\psi^{p-2} \mathrm{~g}$ has constant scalar curvature $v$ if and only if $\psi$ satisfies the Yamabe equation

$$
a \Delta \psi+S \psi=\nu|\psi|^{p-1} .
$$

We can show, using Hölder inequality, that $Q$ is bounded below on a conformal class. So we define

$$
\lambda([g])=\inf \{Q(\tilde{g}): \tilde{g} \text { is conformal to } g\} .
$$

This constant $\lambda([g])$ is an invariant of the conformal class $[g]$ of $g$ on $M$, called the Yamabe invariant.

The YAMABe PROblem [14]. Given a compact Riemannian $n$-manifold $(M, g)$, find a metric $\tilde{g}$ conformal to $g$ which minimizes $Q$ on $[g]$, so that $Q(\tilde{g})=\lambda([g])$. Then $\tilde{g}$ is a stationary point of $Q$ on $[g]$, and so has constant scalar curvature.

A solution $\tilde{g}$ of the Yamabe problem is called a Yamabe metric. The problem was posed in 1960 by Yamabe [14] who gave a proof that such a metric $\tilde{g}$ always exists. His idea was to choose a minimizing sequence for $Q$ on $[g]$ and show that a subsequence converges to a smooth minimizer $\tilde{g}$ for $Q$. Unfortunately, the proof contained an error, discovered by Trudinger [12]. The proof was eventually repaired by Trudinger, Aubin, Schoen, and Yau.

If $\lambda([g]) \leq 0$, then constant scalar curvature metrics in $[g]$ are unique up to homothety [1, page 135] and are Yamabe metrics. Thus, if $\lambda([g])<0$, then $[g]$ contains a unique metric $\tilde{g}$ with scalar curvature -1 , and if $\lambda([g])=0$, then $[g]$ contains a unique metric $\tilde{g}$ with scalar curvature 0 and volume 1 . However, if $\lambda([g])>0$, then constant scalar curvature metrics in $[g]$ are not necessarily unique up to homothety, and may be higher stationary points of $Q$ rather than Yamabe metrics. Thus, $[g]$ may contain several different metrics with scalar curvature 1. We will see an example of this in Section 3.3.

In Sections 3 and 4, weak solutions in $L_{1}^{2}(M)$ to the Yamabe equation (1.6) will be constructed for certain special compact manifolds $M$. For these solutions to give metrics of constant scalar curvature, it is necessary that they should 
not be just $L_{1}^{2}$ solutions but $C^{\infty}$ solutions. We quote a result from Trudinger [12, Theorem 3, page 271] showing that this is the case.

Proposition 1.1. Let $(M, g)$ be a compact Riemannian $n$-manifold with scalar curvature $S$, and $u$ a weak $L_{1}^{2}$ solution of $a \Delta u+S u=\tilde{S}|u|^{(n+2) /(n-2)}$ for $\tilde{S} \in C^{\infty}(M)$. Then $u \in C^{2}(M)$, and where $u$ is nonzero, it is $C^{\infty}$.

1.3. Stereographic projections. Let $(M, g)$ be a compact Riemannian manifold with positive scalar curvature. Then, by [7, Lemma 6.1, page 63], the operator $a \Delta+S$ in (1.6) admits a Green's function $\Gamma_{m}$ for each $m \in M$, which is unique and strictly positive. That is, $\Gamma_{m}: M \backslash\{m\} \rightarrow(0, \infty)$ is smooth with $(a \Delta+S) \Gamma_{m}=0$ on $M \backslash\{m\}$, and $\Gamma_{m}$ is singular at $m$ with $(a \Delta+S) \Gamma_{m}=\delta_{m}$ on $M$ in the sense of distributions.

DEFINITION 1.2. Let $(M, g)$ be a compact Riemannian manifold with positive scalar curvature. For $m \in M$, define the metric $\hat{g}=\Gamma_{m}^{p-2} g$ on $\hat{M}=M \backslash\{m\}$ where $\Gamma_{m}$ is the Green's function of $a \Delta+S$ at $m$. Then $\hat{g}$ has zero scalar curvature by (1.5). We call $(\hat{M}, \hat{g})$ the stereographic projection of $M$ from $m$.

The stereographic projection of $\mathscr{\varphi}^{n}$ is $\mathbb{R}^{n}$. In general, stereographic projections $(\hat{M}, \hat{g})$ are asymptotically flat, that is, their noncompact ends resemble $\mathbb{R}^{n}$ in a more precise way in [7, Definition 6.3]. Following Lee and Parker [7, page 64], we use the notation that $\phi=O^{\prime}\left(|x|^{q}\right)$ means $\phi=O\left(|x|^{q}\right)$ and $\nabla \phi=O\left(|x|^{q-1}\right)$, and $\phi=O^{\prime}\left(|x|^{q}\right)$ means $\phi=O\left(|x|^{q}\right), \nabla \phi=O\left(|x|^{q-1}\right)$, and $\nabla^{2} \phi=O\left(|x|^{q-2}\right)$ for small or large $x$ in $\mathbb{R}^{n}$, depending on the context. For simplicity, we will suppose that $g$ is conformally flat near $m$ in $M$. In this case, the asymptotic expansion of the metric $\hat{g}$ is particularly simple, [7, Theorem 6.5(a)].

Proposition 1.3. Let $(M, g)$ be a compact Riemannian manifold with positive scalar curvature, and suppose $m \in M$ has a neighbourhood that is conformally flat. Let $(\hat{M}, \hat{g})$ be the stereographic projection of $M$ from $m$. Then there exist $\mu \in \mathbb{R}$ and asymptotic coordinates $\left\{x^{i}\right\}$ on $\hat{M}$ with respect to $\hat{g}_{i j}$ satisfing

$$
\hat{g}_{i j}(x)=\psi^{p-2}(x) \delta_{i j}
$$

where $\psi(v)=1+\mu|x|^{2-n}+O^{\prime \prime}\left(|x|^{1-n}\right)$ for large $|x|$.

This constant $\mu$ is proportional to an important invariant of asymptotically flat manifolds called the mass, which is defined and studied by Bartnik [2]. For physical reasons, it was conjectured that the mass must be nonnegative for complete asymptotically flat manifolds of nonnegative scalar curvature, and zero only for flat space. This was proved for spin manifolds by Witten [13] whose proof was generalized to $n$ dimensions by Bartnik [2, Section 6].

A proof of the general case when $n \leq 7$ is given by Schoen [10, Section 4]. He also claims the result for all dimensions [9, page 481] and [10, page 145], but 
the proof has not been published. This "positive mass theorem" in dimensions 3 , 4, and 5 was important in the completion of the proof of the Yamabe problem by Schoen [9]. We will only need the case when the metric $g$ of $M$ is conformally flat about $m$, which we state in the following theorem.

THEOREM 1.4. In the situation of Proposition 1.3 (perhaps with the additional assumption that $M$ is spin or $n \leq 7)$, we have $\mu \geq 0$ with equality if and only if $M$ is $\varphi^{n}$, and its metric is conformal to the round metric.

2. Glued metrics on connected sums. In this section, we will define two families of metrics $g_{t}$ on the connected sum $M$ of constant scalar curvature Riemannian manifolds $\left(M^{\prime}, g^{\prime}\right)$ and $\left(M^{\prime \prime}, g^{\prime \prime}\right)$. The first family, in Section 2.1, is made by choosing $M^{\prime}$ of constant scalar curvature $v$, cutting out a small ball, and gluing in a stereographic projection of $M^{\prime \prime}$, homothetically shrunk very small. The second family, in Section 2.2, is made by choosing $M^{\prime}$ and $M^{\prime \prime}$ both of constant scalar curvature $v$, and joining them by a small "neck" of zero scalar curvature. The relation between these families, when $v=1$, is discussed in Section 2.3.

We finish with two results, Propositions 2.1 and 2.2, about the families of metrics. The first gives explicit bounds for their scalar curvature to determine how good an approximation to constant scalar curvature they are. The second shows that the Sobolev constant for a certain Sobolev embedding of function spaces can be given a bound independent of the width of the neck, for small values of this parameter.

2.1. Combining constant and positive scalar curvature. Let $\left(M^{\prime}, g^{\prime}\right)$ be a compact Riemannian $n$-manifold with constant scalar curvature $v$. Applying a homothety to $g^{\prime}$ if necessary, we may assume that $v=1,0$, or -1 . For simplicity, assume that $m^{\prime} \in M^{\prime}$ has a neighbourhood in which $g^{\prime}$ is conformally flat; this assumption will be dropped in Section 3.4. Then $M^{\prime}$ contains a ball $B^{\prime}$ about $m^{\prime}$, with a diffeomorphism $\Phi^{\prime}$ from $B_{\delta}(0) \subset \mathbb{R}^{n}$ to $B^{\prime}$ for some $\delta \in(0,1)$, such that $\Phi^{\prime}(0)=m^{\prime}$ and $\left(\Phi^{\prime}\right)^{*}\left(g^{\prime}\right)=\left(\psi^{\prime}\right)^{p-2} h$ for some function $\psi^{\prime}$ on $B_{\delta}(0)$, where $h$ is the standard metric on $\mathbb{R}^{n}$. By choosing a different conformal identification with $B_{\delta}(0)$ if necessary, we may suppose $\psi^{\prime}(0)=1$ and $d \psi^{\prime}(0)=0$, so that $\psi^{\prime}(v)=1+O^{\prime}\left(|v|^{2}\right)$ in the notation of Section 1.3.

Let $\left(M^{\prime \prime}, g^{\prime \prime}\right)$ be a compact Riemannian $n$-manifold with scalar curvature 1 . As with $M^{\prime}$, suppose $m^{\prime \prime} \in M^{\prime \prime}$ has a neighbourhood in which $g^{\prime \prime}$ is conformally flat. Let $(\hat{M}, \hat{g})$ be the stereographic projection of $M^{\prime \prime}$ from $m^{\prime \prime}$, as in Definition 1.2 , so that $\hat{M}=M^{\prime \prime} \backslash\left\{m^{\prime \prime}\right\}$, and $\hat{g}$ is asymptotically flat with zero scalar curvature, and is conformal to $g^{\prime \prime}$.

By Proposition 1.3, there is an immersion $\Xi^{\prime \prime}: \mathbb{R}^{n} \backslash \bar{B}_{R}(0) \rightarrow \hat{M}$ for some $R>0$ whose image is the complement of a compact set in $\hat{M}$, such that $\left(\Xi^{\prime \prime}\right) *(\hat{g})=$ $\xi^{p-2} h$ where $\xi$ is a smooth function on $\mathbb{R}^{n} \backslash \bar{B}_{R}(0)$ satisfying $\xi(v)=1+O^{\prime} \times$ $\left(|v|^{2-n}\right)$. By making $\delta$ smaller if necessary, we set $R=\delta^{-4}$. 
A family of metrics $\left\{g_{t}: t \in(0, \delta)\right\}$ on $M=M^{\prime} \# M^{\prime \prime}$ will now be written down. For any $t \in(0, \delta)$, define $M$ and the conformal class of $g_{t}$ by

$$
M=\left(M^{\prime} \backslash \Phi^{\prime}\left[\bar{B}_{t^{2}}(0)\right]\right) \amalg\left(\hat{M} \backslash\left(\Xi^{\prime \prime}\left[\mathbb{R}^{n} \backslash B_{t^{-5}}(0)\right]\right)\right) /{ }_{t},
$$

where $\sim_{t}$ is the equivalence relation defined by

$$
\Phi^{\prime}[v] \sim{ }_{t} \Xi^{\prime \prime}\left[t^{-6} v\right] \quad \text { whenever } v \in \mathbb{R}^{n}, t^{2}<|v|<t .
$$

The conformal class $\left[g_{t}\right]$ of $g_{t}$ is then given by the restriction of the conformal classes of $g^{\prime}$ and $\hat{g}$ to the open sets of $M^{\prime}$ and $M^{\prime \prime}$ that make up $M$; this definition makes sense because the conformal classes agree on the annulus of overlap where the two open sets are glued by $\sim_{t}$. Let $A_{t}$ be this annulus in $M$. Then $A_{t}$ is diffeomorphic via $\Phi^{\prime}$ to the annulus $\left\{v \in \mathbb{R}^{n}: t^{2}<|v|<t\right\}$ in $\mathbb{R}^{n}$.

To define a metric $g_{t}$ within the conformal class just given, take $g_{t}=g^{\prime}$ on the component of $M \backslash A_{t}$ coming from $M^{\prime}$, and $g_{t}=t^{12} \hat{g}$ on the component coming from $\hat{M}$. It remains to choose a conformal factor on $A_{t}$ which is identified with $\left\{v \in \mathbb{R}^{n}: t^{2}<|v|<t\right\}$ by $\Phi^{\prime}$. Here we set $\left(\Phi^{\prime}\right)^{*}\left(g_{t}\right)=\psi_{t}^{p-2} h$ where $\psi_{t}$ is defined below.

The conditions for smoothness at the edges of the annulus $A_{t}$ are that $\psi_{t}(v)$ should join smoothly onto $\psi^{\prime}(v)$ at $|v|=t$, and onto $\xi\left(t^{-6} v\right)$ at $|v|=t^{2}$. Choose a $C^{\infty}$-function $\sigma: \mathbb{R} \rightarrow[0,1]$, that is, 0 for $x \geq 2$ and 1 for $x \leq 1$ and strictly decreasing in $[1,2]$. Let $\beta_{1}(v)=\sigma(\log |v| / \log t)$ and $\beta_{2}(v)=1-\beta_{1}(v)$ for all $v \in \mathbb{R}^{n}$ with $t^{2}<|v|<t$. Finally, define $\psi_{t}$ by

$$
\psi_{t}(v)=\beta_{1}(v) \psi^{\prime}(v)+\beta_{2}(v) \xi\left(t^{-6} v\right) .
$$

This ends the definition of $g_{t}$ for $t \in(0, \delta)$. The reasoning behind the definition-why $\hat{g}$ is shrunk by a factor of $t^{6}$, but the cutoff functions change between radii $t$ and $t^{2}$, for instance-will emerge in Section 2.4, where we show that for this definition, the scalar curvature of $g_{t}$ is close to the constant function $v$ in the $L^{n / 2}$-norm.

2.2. Combining two metrics of constant scalar curvature $v$. A family of metrics will now be defined on the connected sum of two Riemannian manifolds with constant scalar curvature $v$. We will do this by writing down a zero scalar curvature Riemannian manifold with two asymptotically flat ends, and gluing one end into each of the constant scalar curvature manifolds using the method of Section 2.1; the new manifold will form the neck in between.

Let $N$ be $\mathbb{R}^{n} \backslash\{0\}$ with metric $g_{N}=\left(1+|v|^{-(n-2)}\right)^{p-2} h$. Then $N$ has two ends and $g_{N}$ is asymptotically flat at each end. The involution $v \mapsto v /|v|^{2}$ is an isometry. Also, as $\Delta|v|^{-(n-2)}=0,(1.5)$ shows that $g_{N}$ has zero scalar curvature.

Let $\left(M^{\prime}, g^{\prime}\right)$ and $\left(M^{\prime \prime}, g^{\prime \prime}\right)$ be Riemannian $n$-manifolds with constant scalar curvature $v$; applying homotheties if necessary, we will assume that $v=1,0$ or 
-1 . We will use the gluing method of Section 2.1 to glue the two asymptotically flat ends of $\left(N, g_{N}\right)$ into $\left(M^{\prime}, g^{\prime}\right)$ and $\left(M^{\prime \prime}, g^{\prime \prime}\right)$. Let $M=M^{\prime} \# M^{\prime \prime}$. A family of metrics $\left\{g_{t}: t \in(0, \delta)\right\}$ on $M$ will be defined, such that $g_{t}$ resembles the union of $\left(M^{\prime}, g^{\prime}\right)$ and $\left(M^{\prime \prime}, g^{\prime \prime}\right)$ joined by a small neck of radius $t^{6}$, modelled upon $\left(N, t^{12} g_{N}\right)$. It will be done briefly, as the treatment generalizes Section 2.1.

Suppose that $M^{\prime}, M^{\prime \prime}$ contain points $m^{\prime}, m^{\prime \prime}$ with neighbourhoods in which $g^{\prime}, g^{\prime \prime}$ are conformally flat. (In Section 3.4 this assumption will be dropped.) Thus $M^{\prime}, M^{\prime \prime}$ contain open balls $B^{\prime}, B^{\prime \prime}$ with diffeomorphisms $\Phi^{\prime}$, $\Phi^{\prime \prime}$ from $B_{\delta}(0)$ in $\mathbb{R}^{n}$ to $B^{\prime}, B^{\prime \prime}$, such that $\Phi^{\prime}(0)=m^{\prime}, \Phi^{\prime \prime}(0)=m^{\prime \prime}$ and $\left(\Phi^{\prime}\right)^{*}\left(g^{\prime}\right)=\left(\psi^{\prime}\right)^{p-2} h$, $\left(\Phi^{\prime \prime}\right) *\left(g^{\prime \prime}\right)=\left(\psi^{\prime \prime}\right)^{p-2} h$, for some functions $\psi^{\prime}, \psi^{\prime \prime}$ on $B_{\delta}(0)$. By choosing different conformal identifications with $B_{\delta}(0)$ if necessary, we may suppose that $\psi^{\prime}(0)=\psi^{\prime \prime}(0)=1$ and $d \psi^{\prime}(0)=d \psi^{\prime \prime}(0)=0$ so that $\psi^{\prime}(v)=1+O^{\prime}\left(|v|^{2}\right)$ and $\psi^{\prime \prime}(v)=1+O^{\prime}\left(|v|^{2}\right)$.

For any $t \in(0, \delta)$, define $M$ and the conformal class of $g_{t}$ by

$$
M=\left(M^{\prime} \backslash \Phi^{\prime}\left[\bar{B}_{t^{2}}(0)\right]\right) \amalg\left(M^{\prime \prime} \backslash \Phi^{\prime \prime}\left[\bar{B}_{t^{2}}(0)\right]\right) \amalg\left\{v \in N: t^{5}<|v|<t^{-5}\right\} / \sim_{t},
$$

where $\sim_{t}$ is the equivalence relation defined by

$$
\begin{gathered}
\Phi^{\prime}\left[t^{6} v\right] \sim{ }_{t} v \quad \text { if } v \in N, t^{-4}<|v|<t^{-5}, \\
\Phi^{\prime \prime}\left[\frac{t^{6} v}{|v|^{2}}\right] \sim{ }_{t} v \quad \text { if } v \in N, t^{5}<|v|<t^{4} .
\end{gathered}
$$

The conformal class $\left[g_{t}\right]$ of $g_{t}$ is then given by the restriction of the conformal classes of $g^{\prime}, g^{\prime \prime}$, and $g_{N}$ to the open sets of $M^{\prime}, M^{\prime \prime}$, and $N$ that make up $M$; this definition makes sense because the conformal classes agree on the annuli of overlap where the three open sets are glued by $\sim_{t}$. Let $A_{t}$ be this region of gluing in $M$. Then $A_{t}$ is diffeomorphic via $\Phi^{\prime}$ and $\Phi^{\prime \prime}$ to two copies of the annulus $\left\{v \in \mathbb{R}^{n}: t^{2}<|v|<t\right\}$.

To define a metric $g_{t}$ within this conformal class, let $g_{t}=g^{\prime}$ on the component of $M \backslash A_{t}$ coming from $M^{\prime}, g_{t}=g^{\prime \prime}$ on the component of $M \backslash A_{t}$ coming from $M^{\prime \prime}$, and $g_{t}=t^{12} g_{N}$ on the component on $M \backslash A_{t}$ coming from $N$. So, it remains to choose a conformal factor on $A_{t}$ itself. Using $\Phi^{\prime}$ and $\Phi^{\prime \prime}$, this is the same as choosing a conformal factor for two copies of the subset $\left\{v \in \mathbb{R}^{n}: t^{2}<|v|<t\right\}$ of $\mathbb{R}^{n}$.

As in Section 2.1, define a partition of unity $\beta_{1}, \beta_{2}$ on $A_{t}$ and define $\psi_{t}$ by $\psi_{t}(v)=\beta_{1}(v) \psi^{\prime}(v)+\beta_{2}(v)\left(1+t^{6(n-2)}|v|^{-(n-2)}\right)$ on the component of $A_{t}$ coming from $M^{\prime}$, and $\psi_{t}(v)=\beta_{1}(v) \psi^{\prime \prime}(v)+\beta_{2}(v)\left(1+t^{6(n-2)}|v|^{-(n-2)}\right)$ on the component coming from $M^{\prime \prime}$. Here $\psi_{t}$ is thought of as a function on $A_{t}$, which is identified by $\Phi^{\prime}$ and $\Phi^{\prime \prime}$ with two disjoint copies of $\left\{v \in \mathbb{R}^{n}: t^{2}<|v|<t\right\}$. Now let $g_{t}$ be $\psi_{t}^{p-2} h$ in $A_{t}$, where $h$ is the push-forward to $A_{t}$ by $\Phi^{\prime}, \Phi^{\prime \prime}$ of the standard metric on $\left\{v \in \mathbb{R}^{n}: t^{2}<|v|<t\right\}$. This completes the definition of $g_{t}$. 
2.3. The relationship between Sections 2.1 and 2.2 when $v=1$. Suppose that $\left(M^{\prime}, g^{\prime}\right)$ and $\left(M^{\prime \prime}, g^{\prime \prime}\right)$ are compact Riemannian $n$-manifolds with scalar curvature 1 , and $m^{\prime}, m^{\prime \prime}$ are points in $M^{\prime}, M^{\prime \prime}$ with conformally flat neighbourhoods. Then Sections 2.1 and 2.2 with $v=1$ define two families of metrics $\left\{g_{t}: t \in(0, \delta)\right\}$ on $M=M^{\prime} \# M^{\prime \prime}$. Reversing the roles of $M^{\prime}$ and $M^{\prime \prime}$ in Section 2.1, we get a third family by shrinking the stereographic projection of $\left(M^{\prime}, g^{\prime}\right)$ from $m^{\prime}$ by a factor $t^{12}$ and gluing it into $\left(M^{\prime \prime}, g^{\prime \prime}\right)$. What is the relationship between these three families of metrics on $M$ ?

Encoded in the choice of maps $\Phi^{\prime}, \Phi^{\prime \prime}$, and $\Xi^{\prime \prime}$ above is a choice of isometric isomorphism $T_{m^{\prime}} M^{\prime} \cong T_{m^{\prime \prime}} M^{\prime \prime}$. Because of the simplifying assumption of conformal flatness near $m^{\prime}, m^{\prime \prime}$, it turns out that this isomorphism is the only arbitrary choice involved in the construction of the conformal classes $\left[g_{t}\right]$, and if we choose the same isomorphism $T_{m^{\prime}} M^{\prime} \cong T_{m^{\prime \prime}} M^{\prime \prime}$ in the three cases, then the three families actually define the same 1-parameter family of conformal classes $\left\{\left[g_{t}\right]: t \in(0, \delta)\right\}$ for small $t$.

However, the parametrization of the conformal classes by $t$ differs in Sections 2.1 and 2.2. Identifying $M^{\prime}$ and $M^{\prime \prime}$ with $\mathbb{R}^{n}$ conformally near $m^{\prime}, m^{\prime \prime}$, and reasoning from the definition of stereographic projection, we find that in Section 2.1, $\sim_{t}$ identifies $v^{\prime} \in M^{\prime}$ with $v^{\prime \prime} \in M^{\prime \prime}$ only if $\left|v^{\prime}\right| \cdot\left|v^{\prime \prime}\right|=t^{6}$, but in Section 2.2, $\sim{ }_{t}$ identifies $v^{\prime} \in M^{\prime}$ with $v^{\prime \prime} \in M^{\prime \prime}$ only if $\left|v^{\prime}\right| \cdot\left|v^{\prime \prime}\right|=t^{12}$.

Because of this, the conformal class $\left[g_{t}\right]$ constructed in Section 2.2 is the same as the conformal class $\left[g_{t^{2}}\right]$ constructed in Section 2.1. We adopted this mildly inconvenient convention because with it, we will be able to prove results simultaneously for the metrics of Sections 2.1 and 2.2 without changing the powers of $t$ involved.

2.4. Estimating the scalar curvature of $g_{t}$. Now we show that the scalar curvature of $g_{t}$ approaches the constant value $v$ in the $L^{n / 2}$-norm as $t \rightarrow 0$.

Proposition 2.1. Let $\left\{g_{t}: t \in(0, \delta)\right\}$ be one of the families of metrics defined on $M=M^{\prime} \# M^{\prime \prime}$ in Sections 2.1 and 2.2. Let the scalar curvature of $g_{t}$ be $v-\epsilon_{t}$. Then there exist $Y, Z>0$ such that $\left|\epsilon_{t}\right| \leq Y$ and ${ }^{g}\left\|\epsilon_{t}\right\|_{L^{n / 2}} \leq Z t^{2}$ for $t \in(0, \delta)$.

Proof. The proof will be given for the metrics $g_{t}$ of Section 2.1 only, the modifications for Section 2.2 being left to the reader. We first derive an expression for $\epsilon_{t}$. Outside $A_{t}$, the scalar curvature of $g_{t}$ is $v$ and 0 on the regions coming from $M^{\prime}$ and $M^{\prime \prime}$. On $A_{t}$, calculating with (1.5) gives

$$
\begin{aligned}
v-\epsilon_{t}(v)= & v \beta_{1}(v)\left(\psi^{\prime}(v)\right)^{(n+2) /(n-2)} \psi_{t}^{-(n+2) /(n-2)}(v) \\
& +a \psi_{t}^{-(n+2) /(n-2)}(v)\left(\Delta \beta_{1}(v)\right)\left(\psi^{\prime}(v)-\xi\left(t^{-6} v\right)\right) \\
& -2 a \psi_{t}^{-(n+2) /(n-2)}(v)\left(\nabla \beta_{1}(v)\right) \cdot\left(\nabla\left(\psi^{\prime}(v)-\xi\left(t^{-6} v\right)\right)\right),
\end{aligned}
$$

since $\beta_{1}+\beta_{2}=1$. 
As $\psi^{\prime}(v)=1+O^{\prime}\left(|v|^{2}\right), \xi(v)=1+O^{\prime}\left(|v|^{2-n}\right)$, and $t^{2}<|v|<t$, it follows easily that $\psi^{\prime}(v)-\xi\left(t^{-6} v\right)=O^{\prime}\left(|v|^{2}\right)$. The reason for choosing to scale $\hat{g}$ by a factor of $t^{12}$ whilst making $\beta_{1}$ change between $t^{2}$ and $t$ is to make this estimate work - the first power has to be as high as 12 to work in dimension 3. Substituting it into (2.6) gives

$$
\begin{aligned}
\left|\epsilon_{t}\right| \leq & |v| \cdot\left|\beta_{1}\left(\psi^{\prime}\right)^{(n+2) /(n-2)} \psi_{t}^{-(n+2) /(n-2)}-1\right| \\
& +\psi_{t}^{-(n+2) /(n-2)} \cdot\left\{\left|\nabla \beta_{1}\right| O(|v|)+\left|\Delta \beta_{1}\right| O\left(|v|^{2}\right)\right\} .
\end{aligned}
$$

Using a lower bound for $\psi^{\prime}$, we find that on the subannulus $t^{2}<|v|<t$, the estimate $\left|\psi_{t}(v)\right| \geq C_{0}>0$ holds for some $C_{0}$ and all $t \in(0, \delta)$. Using this to get rid of the $\psi_{t}$ terms on the right-hand side and an upper bound for $\psi^{\prime}$, it can be seen that

$$
\left|\epsilon_{t}\right|=O(1)+\left|\nabla \beta_{1}\right| O(|v|)+\left|\Delta \beta_{1}\right| O\left(|v|^{2}\right)
$$

on the subannulus $t^{2}<|v|<t$. But

$$
\left|\nabla \beta_{1}\right|=\frac{\left|d \tau_{1} / d x\right|}{|v \log t|}=O\left(|v|^{-1}\right)
$$

and in a similar way $\left|\Delta \beta_{1}\right|=O\left(|v|^{-2}\right)$. Substituting into (2.8), we find that for all $t \in(0, \delta),\left|\epsilon_{t}\right| \leq Y$ on the subannulus $t^{2}<|v|<t$, for some $Y \geq|v|$.

Thus, $\left|\epsilon_{t}\right| \leq Y$ on $A_{t}$, and outside $A_{t}, \epsilon_{t}=0$ on the component coming from $M^{\prime}$ and $\left|\epsilon_{t}\right|=|\nu| \leq Y$ on the component coming from $M^{\prime \prime}$. Therefore, $\left|\epsilon_{t}\right| \leq Y$ giving the first part of the proposition. To prove the second part, observe that by the estimates on $\psi_{t}$ above, the support of $\epsilon_{t}$ has volume $\leq C_{1} t^{n}$, for some $C_{1}>0$. So, ${ }^{g} t\left\|\epsilon_{t}\right\|_{L^{n / 2}} \leq Z t^{2}$ where $Z=Y C_{1}^{2 / n}$.

2.5. A uniform bound for a Sobolev embedding. If $(M, g)$ is a compact Riemannian $n$-manifold, then $L_{1}^{2}(M)$ is continuously embedded in $L^{2 n /(n-2)}(M)=$ $L^{p}(M)$ by the Sobolev embedding theorem. This means that $L_{1}^{2}(M) \subset L^{p}(M)$, and $\|\phi\|_{L^{p}} \leq A\|\phi\|_{L_{1}^{2}}$ for all $\phi \in L_{1}^{2}(M)$, and some $A>0$ depending on $g$. We will prove that this holds for the metrics $g_{t}$ of Sections 2.1 and 2.2, with $A$ independent of $t$.

Proposition 2.2. Let $\left\{g_{t}: t \in(0, \delta)\right\}$ be one of the families of metrics defined on $M=M^{\prime} \# M^{\prime \prime}$ in Sections 2.1 and 2.2. Then there exist $A>0$ and $\zeta \in(0, \delta)$ such that ${ }^{g t}\|\phi\|_{L^{p}} \leq A \cdot g_{t}\|\phi\|_{L_{1}^{2}}$ whenever $\phi \in L_{1}^{2}(M)$ and $0<t \leq \zeta$.

Proof. First consider the metrics of Section 2.1. The proof works by proving similar Sobolev embedding results on the component manifolds $\left(M^{\prime}, g^{\prime}\right)$ and $\left(\hat{M}, t^{12} \hat{g}\right)$ that make up $\left(M, g_{t}\right)$ and then gluing them together. These are given in the following lemmas, the first follows from the Sobolev embedding theorem [1, Theorem 2.20]. 
LEMMA 2.3. There exists $D_{1}>0$ with $g^{\prime}\|\phi\|_{L^{p}} \leq D_{1} \cdot g^{\prime}\|\phi\|_{L_{1}^{2}}$ for all $\phi \in$ $L_{1}^{2}\left(M^{\prime}\right)$.

LEMmA 2.4. There exists $D_{2}>0$ with $t^{12} \hat{g}\|\phi\|_{L^{p}} \leq D_{2} \cdot t^{12} \hat{g}\|\nabla \phi\|_{L^{2}}$ for all $\phi \in L_{1}^{2}(\hat{M})$ and $t>0$.

Proof. The inequality $\|\phi\|_{L^{p}} \leq D_{2}\|\nabla \phi\|_{L^{2}}$ is invariant under homotheties, and so, it is enough to prove the lemma when $t=1$. Bartnik [2, Sections 1 and 2] reviews the theory of weighted Sobolev spaces on asymptotically flat manifolds. We will apply [2, Theorems 1.2 and 1.3] which hold on asymptotically flat manifolds by [2, page 676]. It was shown by [2, Theorem 1.2(iv)] with $k=1$, $p=q=2$, and $\delta=(2-n) / 2$, that there exists $B>0$ such that

$$
\left(\int_{\hat{M}}|\phi|^{2 n /(n-2)} d V_{\hat{g}}\right)^{(n-2) / n} \leq B\left(\int_{\hat{M}}|\nabla \phi|^{2} d V_{\hat{g}}+\int_{\hat{M}}|\phi|^{2} \sigma^{-2} d V_{\hat{g}}\right)
$$

for all $\phi \in L_{1}^{2}(N)$, where $\sigma: N \rightarrow[1, \infty)$ is defined by $\sigma(x)^{2}=1+d\left(x, x_{0}\right)^{2}$ for some base point $x_{0} \in N$. But [2, Theorem 1.3(i)] with $p=2$ and $\delta=(2-n) / 2$ shows that there exists $C>0$ such that

$$
\int_{\hat{M}}|\phi|^{2} \sigma^{-2} d V_{\hat{g}} \leq C \int_{\hat{M}}|\nabla \phi|^{2} d V_{\hat{g}}
$$

Combining (2.10) and (2.11) and taking square roots proves the lemma, with $D_{2}=(B(1+C))^{1 / 2}$.

The volume form of $g_{t}$ on $A_{t}$ is $\psi_{t}^{p} d V_{h}$, so the contribution from $A_{t}$ to $\int_{M} \phi^{p} d V_{g_{t}}$ is $\int_{A_{t}}\left(\psi_{t} \phi\right)^{p} d V_{h}$. Using (2.3), we may eliminate $\psi_{t}$, divide into integrals on the component manifolds, and show that $g_{t}\|\phi\|_{L^{p}} \leq g^{\prime}\left\|\beta_{1} \phi\right\|_{L^{p}}+$ $t^{12} \hat{g}\left\|\beta_{2} \phi\right\|_{L^{p}}$ for $\phi \in L_{1}^{2}(M)$. Now

$$
\begin{aligned}
g^{\prime}\left\|\beta_{1} \phi\right\|_{L_{1}^{2}} & \leq{ }^{g^{\prime}}\left\|\beta_{1} \phi\right\|_{L^{2}}+{ }^{g^{\prime}}\left\|\beta_{1} \nabla \phi\right\|_{L^{2}}+{ }^{g^{\prime}}\left\|\phi\left|\nabla \beta_{1}\right|\right\|_{L^{2}} \\
& \leq{ }^{g^{\prime}}\left\|\beta_{1} \phi\right\|_{L^{2}}+{ }^{g^{\prime}}\left\|\beta_{1} \nabla \phi\right\|_{L^{2}}+{ }^{g^{\prime}}\left\|\left.\phi\right|_{A_{t}}\right\|_{L^{p}} \cdot g^{\prime}\left\|\left.d \beta_{1}\right|_{A_{t}}\right\|_{L^{n}},
\end{aligned}
$$

by Hölder inequality. But as $\left\|\left.d \beta_{1}\right|_{A_{t}}\right\|_{L^{n}}$ is a conformally invariant norm, we have ${ }^{g^{\prime}}\left\|\left.d \beta_{1}\right|_{A_{t}}\right\|_{L^{n}}={ }^{h}\left\|\left.d \beta_{1}\right|_{A_{t}}\right\|_{L^{n}}$, where $h$ is the standard metric on $\mathbb{R}^{n}$.

Combining Lemmas 2.3 and 2.4, (2.12), and the obvious analogue of (2.12) for $\hat{M}$, and remembering that $d \beta_{1}+d \beta_{2}=0$, we obtain

$$
\begin{aligned}
g_{t}\|\phi\|_{L^{p}} \leq & D_{1} \cdot g^{\prime}\left\|\beta_{1} \phi\right\|_{L_{1}^{2}}+D_{2} \cdot{ }^{12} \hat{g}\left\|\beta_{2} \phi\right\|_{L_{1}^{2}} \\
\leq & D_{1}\left({ }^{g^{\prime}}\left\|\beta_{1} \phi\right\|_{L^{2}}+{ }^{g^{\prime}}\left\|\beta_{1} \nabla \phi\right\|_{L^{2}}+{ }^{g^{\prime}}\left\|\left.\phi\right|_{A_{t}}\right\|_{L^{p}} \cdot h\left\|\left.d \beta_{1}\right|_{A_{t}}\right\|_{L^{n}}\right) \\
& +D_{2}\left(t^{12} \hat{g}\left\|\beta_{2} \phi\right\|_{L^{2}}+{ }^{12} \hat{g}\left\|\beta_{2} \nabla \phi\right\|_{L^{2}}+{ }^{{ }^{12} \hat{g}}\left\|\left.\phi\right|_{A_{t}}\right\|_{L^{p}} \cdot h\left\|\left.d \beta_{1}\right|_{A_{t}}\right\|_{L^{n}}\right) \\
\leq & D_{3}{ }^{g t}\|\phi\|_{L_{1}^{2}}+D_{4} \cdot{ }^{t}\|\phi\|_{L^{p}} \cdot h\left\|\left.d \beta_{1}\right|_{A_{t}}\right\|_{L^{n}}
\end{aligned}
$$


for all $t \in(0, \delta)$ and some $D_{3}, D_{4}>0$ depending on $D_{1}, D_{2}$ and bounds for the ratios of the various conformal factors on $A_{t}$.

From (2.13), we see that Proposition 2.2 holds with $A=2 D_{3}$ provided that $D_{4} \cdot{ }^{h}\left\|\left.d \beta_{1}\right|_{A_{t}}\right\|_{L^{n}} \leq 1 / 2$. As $\beta_{1}$ depends only on $r=|v|$, we have

$$
\left.{ }^{h}\left|d \beta_{1} \|_{L^{n}}^{n}=\omega_{n-1} \cdot \int_{t^{2}}^{t} r^{n-1}\right| \frac{d \beta_{1}}{d r}\right|^{n} d r=\omega_{n-1}|\log t|^{1-n} \cdot \int_{1}^{2}\left|\frac{d \tau_{i}}{d x}\right|^{n} d x,
$$

where $\omega_{n-1}$ is the volume of the unit sphere $\varphi^{n-1}$ in $\mathbb{R}^{n}$. So when $t$ is small, ${ }^{h}\left\|d \beta_{1}\right\|_{L^{n}}$ is small, and there exists $\zeta \in(0, \delta)$ such that $D_{4}{ }^{h}\left\|\left.d \beta_{1}\right|_{A_{t}}\right\|_{L^{n}} \leq 1 / 2$ when $0<t \leq \zeta$. This completes the proposition for the metrics of Section 2.1. The proof for the metrics of Section 2.2 requires only simple modifications, and we leave it to the reader.

3. Existence results for scalar curvature \pm 1 . Let $M$ be the manifold of Sections 2.1 and 2.2 with one of the metrics $g_{t}$ defined there, and denote its scalar curvature by $S$. As in Section 1.2, a conformal change to $\tilde{g}_{t}=\psi^{p-2} g_{t}$ may be made, and the condition for $\tilde{g}_{t}$ to have constant scalar curvature $v$ is the Yamabe equation

$$
a \Delta \psi+S \psi=\nu|\psi|^{p-1}
$$

Now the metrics $g_{t}$ have scalar curvature close to $v$, so let $S=v-\epsilon$; then by Proposition 2.1, ${ }^{g_{t}}\|\epsilon\|_{L^{n / 2}} \leq Z t^{2}$. Also we would like the conformal change to be close to 1 , so put $\psi=1+\phi$, where we aim to make $\phi$ small. Substituting both of these changes into (3.1) gives

$$
a \Delta \phi-v b \phi=\epsilon+\epsilon \phi+\nu f(\phi)
$$

where $b=4 /(n-2)$ and $f(t)=|1+t|^{(n+2) /(n-2)}-1-(n+2) t /(n-2)$.

In this section, we will suppose that $v= \pm 1$, as the zero scalar curvature case requires different analytic treatment and will be considered in Section 4. Equation (3.2) has been written so that on the left is a linear operator $a \Delta-v b$ applied to $\phi$, and on the right are the error terms.

The method of Section 3.1 is to define by induction a sequence $\left\{\phi_{i}\right\}_{i=0}^{\infty}$ of functions in $L_{1}^{2}(M)$ by $\phi_{0}=0$, and

$$
a \Delta \phi_{i}-v b \phi_{i}=\epsilon+\epsilon \phi_{i-1}+v f\left(\phi_{i-1}\right) .
$$

This depends upon being able to invert the operator $a \Delta-v b$, and we consider the existence and size of the inverse in Sections 3.2 and 3.3. Given this invertibility, we show that if $\epsilon$ is sufficiently small, $\left\{\phi_{i}\right\}_{i=0}^{\infty}$ converges to $\phi \in L_{1}^{2}(M)$ which is a weak solution of (3.2). Finally, we show that $\phi$ is smooth and $\psi=$ $1+\phi$ is positive so that $\tilde{g}_{t}$ has constant scalar curvature $v$. 
In Sections 3.2 and 3.3, we state the existence theorems for constant positive and negative scalar curvature respectively on connected sums, the main results of this section. Note that Section 3.3 produces three distinct metrics of scalar curvature 1 in the conformal class of each suitable connected sum of manifolds with scalar curvature 1 , in contrast to the negative scalar curvature case, where any metric of scalar curvature -1 is unique in its conformal class.

3.1. An existence result for constant scalar curvature. Fix $v= \pm 1$ and suppose that $(M, g)$ is a compact Riemannian $n$-manifold. Let $A, B, X$, and $Y$ be positive constants, to be chosen later. We write down four properties, which $(M, g)$ may or may not satisfy.

Property 1. The volume of $M$ satisfies $X / 2 \leq \operatorname{vol}(M) \leq X$.

Property 2. Let the scalar curvature of $g$ be $v-\epsilon$, then $|\epsilon| \leq Y$.

Property 3. Whenever $\phi \in L_{1}^{2}(M), \phi \in L^{p}(M)$, and $\|\phi\|_{L^{p}} \leq A\|\phi\|_{L_{1}^{2}}$.

Property 4. For every $\xi \in L^{2 n /(n+2)}(M)$, there exists a unique $\phi \in L_{1}^{2}(M)$

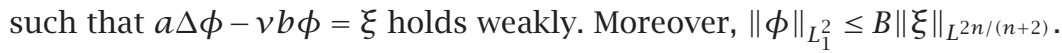

We of course think of $(M, g)$ as being the manifold $M$ of Sections 2.1 and 2.2, with one of the metrics $g_{t}$ defined there. Then, Property 1 is clear from the definitions, Property 2 comes from Proposition 2.1, Property 3 from Proposition 2.2, and Property 4 remains to be proved. In terms of these properties, we state the next result which is the core of the analysis of this section.

THEOREM 3.1. Let $A, B, X, Y>0$ and $n \geq 3$ be given. Then there exist $W, c>0$ depending only upon $A, B, X, Y$, and $n$, such that if $(M, g)$ satisfies Properties 1 , 2,3 , and 4 above and $\|\epsilon\|_{L^{n / 2}} \leq c$, then $g$ admits a smooth conformal rescaling to $\tilde{g}=(1+\phi)^{p-2} g$, with constant scalar curvature $v$, and $\|\phi\|_{L_{1}^{2}} \leq W\|\epsilon\|_{L^{n / 2}}$.

Proof. Suppose that $(M, g)$ satisfies Properties 1, 2, 3, and 4 above. Define a map $T: L_{1}^{2}(M) \rightarrow L_{1}^{2}(M)$ by $T \eta=\xi$ where

$$
a \Delta \xi-v b \xi=\epsilon+\epsilon \eta+v f(\eta) .
$$

By Property 4, $\xi$ exists and is unique provided that the right-hand side is in $L^{2 n /(n+2)}(M)$. So it must be shown that if $\eta \in L_{1}^{2}(M)$, then $\epsilon+\epsilon \eta+v f(\eta) \epsilon$ $L^{2 n /(n+2)}(M)$. Now $\epsilon \in L^{n / 2}(M)$ implies that $\epsilon \in L^{2 n /(n+2)}(M)$; by the Sobolev embedding theorem, $\eta \in L^{2 n /(n-2)}(M)$, and as $\epsilon \in L^{n / 2}(M)$, it follows that $\epsilon \eta \in$ $L^{2 n /(n+2)}(M)$. Thus, the first two terms are in $L^{2 n /(n+2)}(M)$. For the third term, as $\eta \in L^{2 n /(n-2)}(M)$ we have $1+\eta \in L^{2 n /(n-2)}(M)$, and so $(1+\eta)^{(n+2) /(n-2)} \in$ $L^{2 n /(n+2)}(M)$. This deals with the first part of $f(\eta)$ and the last two parts are trivially in $L^{2 n /(n+2)}(M)$. Therefore, the right-hand side of (3.4) is in $L^{2 n /(n+2)}(M)$, and the map $T$ is well defined.

Now define a sequence $\left\{\phi_{i}\right\}_{i=0}^{\infty}$ of elements of $L_{1}^{2}(M)$ by $\phi_{i}=T^{i}(0)$. Our first goal is to prove that if $\|\epsilon\|_{L^{n / 2}}$ is sufficiently small, then this sequence 
converges in $L_{1}^{2}(M)$. Setting $\phi$ as the limit of the sequence, (3.4) implies that $\phi$ will satisfy (3.2), as we would like. This will be achieved via the next lemma.

LEMmA 3.2. Suppose $L$ is a Banach space with norm $\|\cdot\|$, and $T: L \rightarrow L$ is a map satisfying $\|T(0)\| \leq F_{0} s$ and

$$
\begin{aligned}
& \|T(v)-T(w)\| \\
& \quad \leq\|v-w\|\left\{F_{1} s+F_{2}(\|v\|+\|w\|)+F_{3}\left(\|v\|^{4 /(n-2)}+\|w\|^{4 /(n-2)}\right)\right\}
\end{aligned}
$$

for all $v, w \in L$, where $F_{0}, F_{1}, F_{2}, F_{3}, s>0$. Then there exists $W>0$ depending only on $F_{0}, F_{1}, F_{2}, F_{3}$, and $n$, such that if $s$ is sufficiently small, then the sequence $\left\{\phi_{i}\right\}_{i=0}^{\infty}$ defined by $\phi_{i}=T^{i}(0)$ converges to a limit $\phi$ in $L$, satisfying $\|\phi\| \leq W s$.

Proof. Putting $v=\phi_{i-1}$ and $w=0$ into (3.5) gives $\left\|\phi_{i}-T(0)\right\| \leq F_{1} s\left\|\phi_{i-1}\right\|+$ $F_{2}\left\|\phi_{i-1}\right\|^{2}+F_{3}\left\|\phi_{i-1}\right\|^{(n+2) /(n-2)}$, and as $\|T(0)\| \leq F_{0} s$, this implies that $\left\|\phi_{i}\right\| \leq$ $\chi\left(\left\|\phi_{i-1}\right\|\right)$ where $\chi(x)=F_{0} s+F_{1} s x+F_{2} x^{2}+F_{3} x^{(n+2) /(n-2)}$.

From the form of this equation, it is clear that there exists $W>0$ depending only on $F_{0}, F_{1}, F_{2}, F_{3}, n$ such that when $s$ is small, there exists an $x$ with $0<x \leq$ $W s$ and $2 x(x)=x$. Suppose $s$ is small enough so that such an $x$ exists. Now $\phi_{0}=0$ so that $\left\|\phi_{0}\right\| \leq x$, and if $\left\|\phi_{i-1}\right\| \leq x$, then $\left\|\phi_{i}\right\| \leq \chi\left(\left\|\phi_{i-1}\right\|\right) \leq \chi(x) \leq x$. Thus, by induction, $\left\|\phi_{i}\right\| \leq x$ for all $i$.

Put $v=\phi_{i}$ and $w=\phi_{i-1}$ in (3.5). This gives

$$
\left\|\phi_{i+1}-\phi_{i}\right\| \leq\left\|\phi_{i}-\phi_{i-1}\right\| \cdot\left(F_{1} s+2 F_{2} x+2 F_{3} x^{4 /(n-2)}\right)
$$

using the inequality $\left\|\phi_{i}\right\| \leq x$ that we have just proved. Dividing the equation $x=2 \chi(x)$ by $x$ and subtracting some terms, it follows that $1>F_{1} s+2 F_{2} x+$ $2 F_{3} x^{4 /(n-2)}>0$, and so $\left\{\phi_{i}\right\}_{i=0}^{\infty}$ converges by comparison with a geometric series. Let the limit of the sequence be $\phi$. Then as $\left\|\phi_{i}\right\| \leq x \leq W s$ for all $i$, by continuity $\phi$ also satisfies $\|\phi\| \leq W s$.

To apply Lemma 3.2, we must show that $T: L_{1}^{2}(M) \rightarrow L_{1}^{2}(M)$ defined above satisfies the hypotheses. Let $s=\|\epsilon\|_{L^{n / 2}}$. We will define $F_{0}, F_{1}, F_{2}, F_{3}>0$ depending only on $A, B, X$, and $n$, such that (3.5) holds.

Putting $\eta=0$ in (3.4) and applying Properties 1 and 4, we see that

$$
\|T(0)\|_{L_{1}^{2}} \leq B\|\epsilon\|_{L^{2 n /(n+2)}} \leq B \operatorname{vol}(M)^{(n-2) / 2 n}\|\epsilon\|_{L^{n / 2}} \leq B X^{(n-2) / 2 n} s,
$$

so let $F_{0}=B X^{(n-2) / 2 n}$. From the definition of $f$, it can be seen that

$$
|f(x)-f(y)| \leq|x-y| \cdot\left(F_{4}(|x|+|y|)+F_{5}\left(|x|^{4 /(n-2)}+|y|^{4 /(n-2)}\right)\right),
$$


where $F_{4}, F_{5}$ are constants depending only on $n$, and $F_{4}=0$ if $n \geq 6$. Let $\eta_{1}, \eta_{2} \in$ $L_{1}^{2}(M)$, and let $T\left(\eta_{i}\right)=\xi_{i}$. Then subtracting (3.4) applied to $\eta_{2}, \xi_{2}$ from (3.4) applied to $\eta_{1}, \xi_{1}$ gives

$$
(a \Delta-v b)\left(\xi_{1}-\xi_{2}\right)=\epsilon \cdot\left(\eta_{1}-\eta_{2}\right)+v\left(f\left(\eta_{1}\right)-f\left(\eta_{2}\right)\right) .
$$

Applying Property 4 and making various estimates gives

$$
\begin{aligned}
\left\|\xi_{1}-\xi_{2}\right\|_{L_{1}^{2}} \leq B\left(\left\|\epsilon \cdot\left(\eta_{1}-\eta_{2}\right)\right\|_{L^{2 n /(n+2)}}+|v|\left\|f\left(\eta_{1}\right)-f\left(\eta_{2}\right)\right\|_{L^{2 n /(n+2)}}\right) & \\
\leq\left\|\eta_{1}-\eta_{2}\right\|_{L_{1}^{2}} \cdot( & F_{1} s+F_{2}\left(\left\|\eta_{1}\right\|_{L_{1}^{2}}+\left\|\eta_{2}\right\|_{L_{1}^{2}}\right) \\
& \left.+F_{3}\left(\left\|\eta_{1}\right\|_{L_{1}^{2}}^{4 /(n-2)}+\left\|\eta_{2}\right\|_{L_{1}^{2}}^{4 /(n-2)}\right)\right)
\end{aligned}
$$

where $F_{1}=A B, F_{2}=A^{2} B F_{4} X^{(6-n) / 2 n}$ and $F_{3}=A^{(n+2) /(n-2)} B F_{5}$. The calculation uses Hölder inequality, (3.8), Properties 1 and 3, the expression $\|\eta\|_{L^{r}} \leq$ $\|\eta\|_{L^{s}}(\operatorname{vol} M)^{(s-r) / r s}$ when $1 \leq r<s$ and $\eta \in L^{r}(M) \subset L^{s}(M)$, and the fact that $F_{4}=0$ if $n \geq 6$.

This inequality is (3.5) for the operator $T: L_{1}^{2}(M) \rightarrow L_{1}^{2}(M)$. So putting $L=$ $L_{1}^{2}(M)$ and applying Lemma 3.2, there is a constant $W>0$ depending only on $F_{0}, F_{1}, F_{2}, F_{3}$, and $n$, such that if $\|\epsilon\|_{L^{n / 2}}$ is sufficiently small, then the sequence $\left\{\phi_{i}\right\}_{i=0}^{\infty}$ defined by $\phi_{i}=T^{i}(0)$ converges to a limit $\phi$, satisfying $\|\phi\| \leq$ $W\|\epsilon\|_{L^{n / 2}}$. Now $W$ depends only on $F_{0}, \ldots, F_{3}$ and $n$, and these depend only on $n, A, B$, and $X$, so $W$ depends only on $n, A, B$, and $X$. Since $\phi_{i}=T\left(\phi_{i-1}\right)$ and $T$ is continuous, taking the limit gives $\phi=T(\phi)$, so (3.4) shows that $\phi$ satisfies (3.2) weakly. Thus, we have proved the following lemma.

LEMMA 3.3. There exists $W>0$ depending only on $n, A, B$, and $X$, such that if $\|\epsilon\|_{L^{n / 2}}$ is sufficiently small, then there exists $\phi \in L_{1}^{2}(M)$ satisfying (3.2) weakly, with $\|\phi\|_{L_{1}^{2}} \leq W\|\epsilon\|_{L^{n / 2}}$.

Thus, weak solutions $\phi$ of (3.2) do exist for small $\|\epsilon\|_{L^{n / 2}}$, and for these $\psi=1+\phi$ is a weak solution of (1.6). But for $\tilde{g}=\psi^{p-2} g$ to be a metric, we need $\psi$ to be smooth and positive. Proposition 1.1 shows that $\psi \in C^{2}(M)$ and is $C^{\infty}$ wherever it is nonzero, so it remains to show that $\psi>0$.

Examples of manifolds (with negative scalar curvature) can be found for which (1.6) admits solutions that change sign, so there is something to be proved. This difficulty does not arise in the proof of the Yamabe problem, as there $\psi$ is the limit of a minimizing sequence of positive functions, so $\psi \geq 0$ automatically.

We deal with this problem in the following proposition, by proving that if $\psi=1+\phi$ is a solution of (1.6) that is negative somewhere, then $\|\phi\|_{L_{1}^{2}}$ must be at least a certain size. So if $\phi$ is small in $L_{1}^{2}(M)$, then $\psi=1+\phi \geq 0$. We then show that $\psi>0$ using a maximum principle. 
Proposition 3.4. If $\|\phi\|_{L_{1}^{2}}$ is sufficiently small, then $\psi \geq 0$.

Proof. Let $\xi=\min (\psi, 0)$. Then $\xi \in L_{1}^{2}(M)$ and $\int_{M} \xi \Delta \psi d V_{g}=\int_{M}|\nabla \xi|^{2} d V_{g}$ since $\xi \Delta \psi=|\nabla \xi|^{2}+(1 / 2) \Delta \xi^{2}$ and $\int_{M} \Delta \xi^{2} d V_{g}=0$. So, multiplying (1.6) by $\xi$ and integrating over $M$ gives

$$
\int_{M}\left(a|\nabla \xi|^{2}+S \xi^{2}+v|\xi|^{p}\right) d V_{g}=0
$$

as $\xi=-|\xi|$. Also, from Property 3 and Hölder inequality, we have

$$
A\|\xi\|_{L_{1}^{2}} \geq\|\xi\|_{L^{p}} \geq \operatorname{vol}(\operatorname{supp} \xi)^{-1 / n}\|\xi\|_{L^{2}}
$$

Using (3.12) to eliminate $\int_{M} a|\nabla \xi|^{2} d V_{g}$ in (3.11) and using $S \geq v-Y,|v|=1$, we find that

$$
\|\xi\|_{L^{p}}^{p} \geq\left(F_{6} \operatorname{vol}(\operatorname{supp} \xi)^{-2 / n}-F_{7}\right)\|\xi\|_{L^{2}}^{2}
$$

for $F_{6}, F_{7}>0$ depending on $A, Y$, and $n$. Now $\|\xi\|_{L^{2}} \geq \operatorname{vol}(\operatorname{supp} \xi)^{1 / n}\|\xi\|_{L^{p}}$ as above, and so either $\|\xi\|_{L^{p}}=0$ or we may substitute this into (3.13) to get

$$
\|\xi\|_{L^{p}}^{4 /(n-2)} \geq F_{6}-F_{7} \operatorname{vol}(\operatorname{supp} \xi)^{2 / n}
$$

If this holds, then either $\|\xi\|_{L^{p}}^{4 /(n-2)} \geq F_{6} / 2$ or $\operatorname{vol}(\operatorname{supp} \xi) \geq\left(F_{6} / 2 F_{7}\right)^{n / 2}$. Both imply that $\|\phi\|_{L^{p}}$ is bounded below by a positive constant, and by Property 3 , $\|\phi\|_{L_{1}^{2}}$ is bounded below by a positive constant too. Conversely, if $\|\phi\|_{L_{1}^{2}}$ is smaller than this constant, then (3.14) cannot hold and so $\|\xi\|_{L^{p}}=0$, which implies $\psi \geq 0$.

We are now ready to define the constant $c$ in Theorem 3.1. Let $c$ be small enough that three conditions hold:

(1) $\|\epsilon\|_{L^{n / 2}} \leq c$ implies $\|\epsilon\|_{L^{n / 2}}$ is sufficiently small to satisfy Lemma 3.3 so that $\phi$ exists and satisfies $\|\phi\|_{L_{1}^{2}} \leq c W$;

(2) $\|\phi\|_{L_{1}^{2}} \leq c W$ implies $\|\phi\|_{L_{1}^{2}}$ is sufficiently small to satisfy Proposition 3.4 so that $\psi=1+\phi \geq 0$

(3) $\|\phi\|_{L_{1}^{2}} \leq c W$ implies $\|\phi\|_{L_{1}^{2}}$ is sufficiently small that $\phi$ cannot be the constant -1 . (As $X / 2 \leq \operatorname{vol}(M)$ by Property 1 , this depends only on $X$.)

Then $c$ depends only on $n, A, B, X$, and $Y$, as the three conditions each separately do. Thus, if $\|\epsilon\|_{L^{n / 2}} \leq c$, then there exists $\phi$ with $\|\phi\|_{L_{1}^{2}} \leq W\|\epsilon\|_{L^{n / 2}}$ such that $\psi=1+\phi \geq 0$ and satisfies (1.6). By the third condition on $c, \psi$ is not identically zero. By Proposition 1.1, $\psi \in C^{2}(M)$, and is $C^{\infty}$ wherever it is 
nonzero. It remains to show that $\psi>0$ for $\tilde{g}=\psi^{p-2} g$ to be nonsingular and have constant scalar curvature $v$. This we achieve using the strong maximum principle [7, Theorem 2.6].

THEOREM 3.5. Suppose $h$ is a nonnegative smooth function on a connected manifold $M$, and $u \in C^{2}(M)$ satisfies $(\Delta+h) u \geq 0$. If $u$ attains its minimum $m \leq 0$, then $u$ is constant on $M$.

As $M$ is compact and $S$ and $\psi$ are continuous, they are bounded on $M$, and there is a constant $h \geq 0$ such that $S-v \psi^{p-2} \leq h$ on $M$. Now $M$ is connected, and $\psi \in C^{2}(M)$ satisfies (1.6) and is nonnegative, so $\psi$ satisfies $a \Delta \psi+h \psi \geq 0$. Thus, by the strong maximum principle, if $\psi$ attains the minimum value zero, then $\psi$ is identically zero on $M$. But it has already been shown that this is not the case, so $\psi$ cannot be zero anywhere and must be strictly positive. The proof of Theorem 3.1 is therefore complete.

3.2. Constant negative scalar curvature. Now we construct metrics of scalar curvature -1 on connected sums using the results of Section 3.1. Fix $v=-1$ and consider the metrics $g_{t}$ of Sections 2.1 and 2.2. Properties 1, 2, and 3 of Section 3.1 have already been dealt with, so it remains to show that Property 4 holds for the metrics $g_{t}$, and that ${ }^{g_{t}}\left\|\epsilon_{t}\right\|_{L^{n / 2}}$ is small when $t$ is small. As $v=-1$, Property 4 is about the invertibility of $a \Delta+b$, which is simple as the eigenvalues of $\Delta$ are nonnegative.

LEMMA 3.6. Let $\left\{g_{t}: t \in(0, \delta)\right\}$ be one of the families of metrics defined on $M=M^{\prime} \# M^{\prime \prime}$ in Sections 2.1 and 2.2, and let $A, \zeta$ be as in Proposition 2.2. Then for all $t \in(0, \zeta]$ and $\xi \in L^{2 n /(n+2)}(M)$, there exists a unique $\phi \in L_{1}^{2}(M)$ such that $a \Delta \phi+b \phi=\xi$ holds weakly and ${ }^{g_{t}}\|\phi\|_{L_{1}^{2}} \leq B^{g_{t}}\|\xi\|_{L^{2 n /(n+2)}}$, where $B=A / b$.

Proof. As $\Delta$ is selfadjoint and all its eigenvalues are nonnegative, and as $a, b>0$, by some well-known analysis, $a \Delta+b$ has a right inverse, $T$ say, from $L^{2}(M) \rightarrow L^{2}(M)$. Now $M$ is compact and so $L^{2}(M) \subset L^{2 n /(n+2)}(M)$. Let $\xi \in L^{2}(M)$. We may define $\phi \in L^{2}(M)$ by $\phi=T \xi$, and $a \Delta \phi+b \phi=\xi$ will hold weakly.

It must be shown first that $\phi \in L_{1}^{2}(M)$ and that it satisfies the inequality. Since $\phi, \xi \in L^{2}(M), \int_{M} \phi \xi d V_{g_{t}}$ exists, and by subtraction, $\int_{M} \phi \Delta \phi d V_{g_{t}}$ exists as well. This is $\int_{M}|\nabla \phi|^{2} d V_{g_{t}}$ and so $\phi \in L_{1}^{2}(M)$, by definition.

Multiplying the expression above by $\phi$ and integrating gives $a \int_{M}|\nabla \phi|^{2} d V_{g_{t}}+$ $b^{g_{t}\|\phi\|_{L^{2}}^{2}}=\int_{M} \phi \xi d V_{g_{t}}$. As $a>b$, the left-hand side is at least $b^{{ }^{g} t}\|\phi\|_{L_{1}^{2}}^{2}$, and the right-hand side is at most ${ }^{g_{t}}\|\phi\|_{L^{p}} g_{t}\|\xi\|_{L^{2 n /(n+2)}}$ by Hölder inequality since $\phi \in L^{p}(M)$ by the Sobolev embedding theorem. But ${ }^{g t}\|\phi\|_{L^{p}} \leq A^{g_{t}}\|\phi\|_{L_{1}^{2}}$ by Proposition 2.2. Putting all this together gives

$$
b^{g_{t}}\|\phi\|_{L_{1}^{2}}^{2} \leq A^{g_{t}}\|\xi\|_{L^{2 n /(n+2)}}{ }^{g t}\|\phi\|_{L_{1}^{2}},
$$

and dividing by $b^{g_{t}}\|\phi\|_{L_{1}^{2}}$ gives ${ }^{g t}\|\phi\|_{L_{1}^{2}} \leq B^{g_{t}}\|\xi\|_{L^{2 n /(n+2)}}$. 
So far we have worked with $\xi \in L^{2}(M)$ rather than $L^{2 n /(n+2)}(M)$. It has been shown that the operator $T: L^{2}(M) \subset L^{2 n /(n+2)}(M) \rightarrow L_{1}^{2}(M)$ is linear and continuous with respect to the $L^{2 n /(n+2)}$ norm on $L^{2}(M)$ and bounded by $B$. But therefore, by elementary functional analysis, the operator $T$ extends uniquely to a continuous operator on the closure of $L^{2}(M)$ in $L^{2 n /(n+2)}(M)$, that is, $L^{2 n /(n+2)}(M)$ itself. Call this extended operator $\bar{T}$. Then for $\xi \in L^{2 n /(n+2)}(M)$, $\phi=\bar{T} \xi$ is a well-defined element of $L_{1}^{2}(M)$, satisfing $g_{t}\|\phi\|_{L_{1}^{2}} \leq B{ }^{g_{t}}\|\xi\|_{L^{2 n /(n+2)}}$, and $a \Delta \phi+b \phi=\xi$ holds in the weak sense, by continuity. This concludes the proof.

All the previous work now comes together to prove the following two existence theorems for metrics of scalar curvature -1 .

THEOREM 3.7. Let $\left(M^{\prime}, g^{\prime}\right)$ and $\left(M^{\prime \prime}, g^{\prime}\right)$ be compact Riemannian n-manifolds with scalar curvature -1 and 1 , respectively. Suppose $M^{\prime}, M^{\prime \prime}$ contain points $m^{\prime}$, $m^{\prime \prime}$ with neighbourhoods in which $g^{\prime}, g^{\prime \prime}$ are conformally flat.

As in Section 2.1, define the family $\left\{g_{t}: t \in(0, \delta)\right\}$ of metrics on $M=M^{\prime} \# M^{\prime \prime}$. Then there exists $C>0$ such that $g_{t}$ admits a smooth conformal rescaling to $\tilde{g}_{t}=(1+\phi)^{p-2} g_{t}$ with scalar curvature -1 for small $t$, and ${ }^{g} t\|\phi\|_{L_{1}^{2}} \leq C t^{2}$.

THEOREM 3.8. Let $\left(M^{\prime}, g^{\prime}\right)$ and $\left(M^{\prime \prime}, g^{\prime \prime}\right)$ be compact Riemannian $n$-manifolds with scalar curvature - 1 . Suppose $M^{\prime}, M^{\prime \prime}$ contain points $m^{\prime}, m^{\prime \prime}$ with neighbourhoods in which $g^{\prime}, g^{\prime \prime}$ are conformally flat.

As in Section 2.2, define the family $\left\{g_{t}: t \in(0, \delta)\right\}$ of metrics on $M=M^{\prime} \# M^{\prime \prime}$. Then there exists $C>0$ such that $g_{t}$ admits a smooth conformal rescaling to $\tilde{g}_{t}=(1+\phi)^{p-2} g_{t}$ with scalar curvature -1 for small $t$, and ${ }^{g_{t}}\|\phi\|_{L_{1}^{2}} \leq C t^{2}$.

The proofs of the theorems are nearly the same, so only the first will be given. To get the second proof, change $\operatorname{vol}\left(M^{\prime}\right)$ to $\operatorname{vol}\left(M^{\prime}\right)+\operatorname{vol}\left(M^{\prime \prime}\right)$ in the definition of $X$.

Proof of TheOrem 3.7. Applying Propositions 2.1 and 2.2 to the family $\left\{g_{t}: t \in(0, \delta)\right\}$ gives a constant $Y$ for Property 2 of Section 3.1, and constants $A, \zeta$ such that if $t \leq \zeta$, then Property 3 holds for $g_{t}$ with constant $A$. By Lemma 3.6, there is a constant $B$ such that Property 4 also holds for $g_{t}$ when $t \leq \zeta$.

It is clear that as $t \rightarrow 0, \operatorname{vol}\left(M, g_{t}\right) \rightarrow \operatorname{vol}\left(M^{\prime}\right)>0$. So there is a constant $X>0$ such that $X / 2 \leq \operatorname{vol}\left(M, g_{t}\right) \leq X$ for small enough $t$. This gives Property 1 . Thus, there are constants $n, A, B, X, Y$ such that Properties $1,2,3$, and 4 of Section 3.1 hold for $\left(M, g_{t}\right)$ when $t$ is small. Theorem 3.1 therefore gives a constant $c$ such that if $g_{t}\left\|\epsilon_{t}\right\|_{L^{n / 2}} \leq c$, we have the smooth conformal rescaling to a constant scalar curvature metric that we want.

But by Proposition 2.1, ${ }^{g t}\left\|\epsilon_{t}\right\|_{L^{n / 2}} \leq Z t^{2}$. So for small enough $t,{ }^{g_{t}}\left\|\epsilon_{t}\right\|_{L^{n / 2}} \leq c$, and there exists a smooth conformal rescaling to a metric $\tilde{g}_{t}=(1+\phi)^{p-2} g_{t}$ 
which has scalar curvature -1 . Moreover, $\|\phi\|_{L_{1}^{2}} \leq W^{g_{t}}\left\|\epsilon_{t}\right\|_{L^{n / 2}} \leq W Z t^{2}$ where $W$ is the constant given by Theorem 3.1. Therefore, putting $C=W Z$ completes the proof.

3.3. Constant positive scalar curvature. Now we construct metrics of scalar curvature 1 on connected sums. The problems we encounter are in proving Property 4 of Section 3.1, which now deals with the invertibility of $a \Delta-b$, and they arise because $a \Delta$ may have eigenvalues close to $b$. Our strategy is to show that if $a \Delta$ has no eigenvalues in a fixed neighbourhood of $b$ on the component manifolds of the connected sum, then for small $t, a \Delta$ has no eigenvalues in a smaller neighbourhood of $b$ on $\left(M, g_{t}\right)$.

This is the content of the next theorem. We will indicate here why the theorem holds, but we leave the proof until the appendix, because it forms a rather long and involved diversion from the main thread of the paper.

THEOREM 3.9. Let $\left\{g_{t}: t \in(0, \delta)\right\}$ be one of the families of metrics defined on $M=M^{\prime} \# M^{\prime \prime}$ in Sections 2.1 and 2.2, and suppose that for some $\gamma>0$, a $\Delta$ has no eigenvalues in $(b-2 \gamma, b+2 \gamma)$ on $\left(M^{\prime}, g^{\prime}\right)$ in Section 2.1, and on both $\left(M^{\prime}, g^{\prime}\right)$ and $\left(M^{\prime \prime}, g^{\prime \prime}\right)$ in Section 2.2. Then, $a \Delta$ has no eigenvalues in $(b-\gamma, b+\gamma)$ on $\left(M, g_{t}\right)$ for small $t$.

Here is a sketch of the proof. Suppose $\phi$ is an eigenvector of $a \Delta$ on $\left(M, g_{t}\right)$ for small $t$. Restricting $\phi$ to the portions of $M$ coming from $M^{\prime}$ and $M^{\prime \prime}$ and smoothing off gives functions on $M^{\prime}$ and $M^{\prime \prime}$. We try to show that one of these is close to an eigenvector of $a \Delta$ on $M^{\prime}$ or $M^{\prime \prime}$. This can be done except when $\phi$ is large on the neck compared to the rest of the manifold.

But as the neck is a small region when $t$ is small, for $\phi$ to be large there and small elsewhere means that $\phi$ must change quickly around the neck, so that $\int_{M}|\nabla \phi|^{2} d V_{g_{t}}$ has to be large compared to $\int_{M} \phi^{2} d V_{g_{t}}$. Thus, the eigenvalue of $\phi$ must be large. Conversely, if the eigenvalue of $\phi$ is close to $b$, then $\phi$ cannot be large on the neck compared to the rest of $M$, and therefore either $M^{\prime}$ or $M^{\prime \prime}$ must also has an eigenvalue close to $b$.

Using this result, Property 4 of Section 3.1 can be proved for the metrics.

LEMMA 3.10. Let $\left\{g_{t}: t \in(0, \delta)\right\}$ be one of the families of metrics defined on $M=M^{\prime} \# M^{\prime \prime}$ in Sections 2.1 and 2.2, and suppose that $b$ is not an eigenvalue of $a \Delta$ on $\left(M^{\prime}, g^{\prime}\right)$ in Section 2.1, and on neither $\left(M^{\prime}, g^{\prime}\right)$ nor $\left(M^{\prime \prime}, g^{\prime \prime}\right)$ in Section 2.2. Then there exists $B>0$ such that for small $t$, whenever $\xi \in$ $L^{2 n /(n+2)}(M)$, there exists a unique $\phi \in L_{1}^{2}(M)$ with $a \Delta \phi-b \phi=\xi$ on $\left(M, g_{t}\right)$, and ${ }^{g_{t}}\|\phi\|_{L_{1}^{2}} \leq B^{g_{t}}\|\xi\|_{L^{2 n /(n+2)}}$.

Proof. The spectrum of $a \Delta$ on a compact manifold is discrete, so if $b$ is not an eigenvalue of $a \Delta$, then $a \Delta$ has no eigenvalues in a neighbourhood of $b$. Suppose $b$ is not an eigenvalue of $a \Delta$ on $\left(M^{\prime}, g^{\prime}\right)$ in Section 2.1, and on neither $\left(M^{\prime}, g^{\prime}\right)$ nor $\left(M^{\prime \prime}, g^{\prime \prime}\right)$ in Section 2.2. Then there exists $\gamma>0$ such that $a \Delta$ has 
no eigenvalues in $(b-2 \gamma, b+2 \gamma)$ on these manifolds. So by Theorem 3.9, $a \Delta$ has no eigenvalues in $(b-\gamma, b+\gamma)$ on $\left(M, g_{t}\right)$ for small $t$.

Thus, easy analytical facts about the Laplacian imply that $a \Delta-b$ has a right inverse $T: L^{2}(M) \rightarrow L^{2}(M)$. As $M$ is compact, $L^{2}(M) \subset L^{2 n /(n+2)}(M)$. Let $\xi \in$ $L^{2}(M)$. Then $\phi=T \xi \in L^{2}(M)$ exists and satisfies the equation $a \Delta \phi-b \phi=\xi$ in the weak sense, and as $a \Delta-b$ has no kernel, $\phi$ is unique. Since $\phi, \xi \in L^{2}(M)$, multiplying this equation by $\phi$ and integrating gives a convergent integral, so by subtraction, $\int_{M}|\nabla \phi|^{2} d V_{g_{t}}$ converges, and $\phi \in L_{1}^{2}(M)$.

It remains to bound $\phi$ in $L_{1}^{2}(M)$. Let $\phi_{1}$ be the part of $\phi$ made up of eigenvectors of $a \Delta$ associated with eigenvalues less than $b$, and $\phi_{2}$ the part associated with eigenvalues greater than $b$. Multiplying the equation $a \Delta \phi-b \phi=\xi$ by $\phi_{2}-\phi_{1}$ and integrating gives

$$
\int_{M}\left(a\left|\nabla \phi_{2}\right|^{2}-b \phi_{2}^{2}\right) d V_{g_{t}}-\int_{M}\left(a\left|\nabla \phi_{1}\right|^{2}-b \phi_{1}^{2}\right) d V_{g_{t}}=\int_{M}\left(\phi_{2}-\phi_{1}\right) \xi d V_{g_{t}}
$$

But the restriction on the eigenvalues of $a \Delta$ means that

$$
\begin{aligned}
& \int_{M} a\left|\nabla \phi_{1}\right|^{2} d V_{g_{t}} \leq(b-\gamma) \int_{M} \phi_{1}^{2} d V_{g_{t}}, \\
& \int_{M} a\left|\nabla \phi_{2}\right|^{2} d V_{g_{t}} \geq(b+\gamma) \int_{M} \phi_{2}^{2} d V_{g_{t}},
\end{aligned}
$$

and these together with (3.16) and Hölder inequality imply that

$$
\frac{\gamma a}{a+b+\gamma} \int_{M}\left(\left|\nabla \phi_{1}\right|^{2}+\left|\nabla \phi_{2}\right|^{2}+\phi_{1}^{2}+\phi_{2}^{2}\right) d V_{g_{t}} \leq{ }^{g t}\left\|\phi_{2}-\phi_{1}\right\|_{L^{p}} g_{t}\|\xi\|_{L^{2 n /(n+2)}} \text {. }
$$

For $t \leq \zeta$, we apply Proposition 2.2 to $\phi_{2}-\phi_{1}$ to give ${ }^{g_{t}}\left\|\phi_{2}-\phi_{1}\right\|_{L^{p}} \leq$

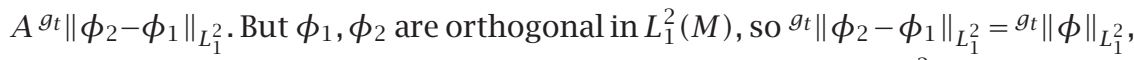
and similarly, the integral on the left-hand side above is ${ }^{g}\|\phi\|_{L_{1}^{2}}^{2}$. Therefore,

$$
\frac{\gamma a}{a+b+\gamma} g_{t}\|\phi\|_{L_{1}^{2}}^{2} \leq A^{g_{t}}\|\phi\|_{L_{1}^{2}} g_{t}\|\xi\|_{L^{2 n /(n+2)}} .
$$

Dividing by $\gamma a^{g_{t}}\|\phi\|_{L_{1}^{2}} /(a+b+\gamma)$, then gives ${ }^{g_{t}}\|\phi\|_{L_{1}^{2}} \leq B^{g_{t}}\|\xi\|_{L^{2 n /(n+2)}}$ for small $t$, where $B=(a+b+\gamma) A / a \gamma$. So the lemma holds for $\xi \in L^{2}(M)$. This may be extended to $\xi \in L^{2 n /(n+2)}(M)$ as in the proof of Lemma 3.6, and the argument is complete.

Now we will prove two existence theorems for metrics of scalar curvature 1 .

THEOREM 3.11. Let $\left(M^{\prime}, g^{\prime}\right)$ and $\left(M^{\prime \prime}, g^{\prime \prime}\right)$ be compact Riemannian n-manifolds with scalar curvature 1. Suppose that $b$ is not an eigenvalue of $a \Delta$ on $\left(M^{\prime}, g^{\prime}\right)$, and that $M^{\prime}, M^{\prime \prime}$ contain points $m^{\prime}, m^{\prime \prime}$ with neighbourhoods in which $g^{\prime}, g^{\prime \prime}$ are conformally flat. 
As in Section 2.1, define the family $\left\{g_{t}: t \in(0, \delta)\right\}$ of metrics on $M=M^{\prime} \# M^{\prime \prime}$. Then there exists $C>0$ such that $g_{t}$ admits a smooth conformal rescaling to $\tilde{g}_{t}=(1+\phi)^{p-2} g_{t}$ with scalar curvature 1 for small $t$, and ${ }^{g t}\|\phi\|_{L_{1}^{2}} \leq C t^{2}$.

THEOREM 3.12. Let $\left(M^{\prime}, g^{\prime}\right)$ and $\left(M^{\prime \prime}, g^{\prime \prime}\right)$ be compact Riemannian $n$-manifolds with scalar curvature 1. Suppose that $b$ is not an eigenvalue of $a \Delta$ on $\left(M^{\prime}, g^{\prime}\right)$ or $\left(M^{\prime \prime}, g^{\prime \prime}\right)$, and that $M^{\prime}, M^{\prime \prime}$ contain points $m^{\prime}, m^{\prime \prime}$ with neighbourhoods in which $g^{\prime}, g^{\prime \prime}$ are conformally flat.

As in Section 2.2, define the family $\left\{g_{t}: t \in(0, \delta)\right\}$ of metrics on $M=M^{\prime} \# M^{\prime \prime}$. Then there exists $C>0$ such that $g_{t}$ admits a smooth conformal rescaling to $\tilde{g}_{t}=(1+\phi)^{p-2} g_{t}$ with scalar curvature 1 for small $t$, and ${ }^{g}\|\phi\|_{L_{1}^{2}} \leq C t^{2}$.

Proof OF TheOREMS 3.11 and 3.12. These are the same as the proofs of Theorems 3.7 and 3.8, except that Lemma 3.10 should be applied in place of Lemma 3.6, and where the proofs of Theorems 3.7 and 3.8 mention scalar curvature -1 , these proofs should have scalar curvature 1 .

In Section 2.3, we saw that when $\mathfrak{g}^{\prime}, \mathfrak{g}^{\prime \prime}$ have scalar curvature 1 , Sections 2.1 and 2.2 define three families of metrics $g_{t}$ on $M=M^{\prime} \# M^{\prime \prime}$ with the same family of conformal classes $\left[g_{t}\right]$ where $t$ in Section 2.2 corresponds to $t^{2}$ in Section 2.1. Thus, Theorems 3.11 and 3.12 construct three different metrics of scalar curvature 1 in the same conformal class $\left[g_{t}\right]$ on $M$, for small $t$. The first resembles $\left(M^{\prime}, g^{\prime}\right)$ with a small, asymptotically flat copy of $M^{\prime \prime}$ glued in at one point, as in Section 2.1. The second is like the first, but swapping $M^{\prime}$ and $M^{\prime \prime}$. The third resembles $\left(M^{\prime}, g^{\prime}\right)$ and $\left(M^{\prime \prime}, g^{\prime \prime}\right)$ joined by a small neck, as in Section 2.2.

These metrics are stationary points of the Hilbert action $Q$, but they need not be absolute minima, that is, Yamabe metrics. The third is never a minimum. If $g^{\prime}, g^{\prime \prime}$ are Yamabe metrics, the author expects that generically one of the first and second metrics will be a Yamabe metric, and in a codimension 1 set of cases when $\operatorname{vol}\left(M^{\prime}\right) \approx \operatorname{vol}\left(M^{\prime \prime}\right)$, both the first and second metrics will be distinct Yamabe metrics.

3.4. Extending to conformally curved metrics. In defining the metrics $\left\{g_{t}\right.$ : $t \in(0, \delta)\}$ in Sections 2.1 and 2.2, we assumed, for simplicity, that $g^{\prime}, g^{\prime \prime}$ are conformally flat in neighbourhoods of $m^{\prime}, m^{\prime \prime}$. It turns out that if we drop this assumption, then provided that the metrics $g_{t}$ on $M$ are suitably defined, the results of Sections 3.2 and 3.3 still hold without change. The principal difference is that the expression (2.6) for the scalar curvature of $g_{t}$ becomes more complicated, with new error terms that have to be estimated and controlled.

Following the method of Section 2.1, we can choose an identification of a ball about $m^{\prime}$ in $M^{\prime}$ with $B_{\delta}(0)$ such that the induced metric on $B_{\delta}(0)$ is $g^{\prime}=$ $h+q^{\prime}$, where $h$ is the standard metric on $\mathbb{R}^{n}$ and $q^{\prime}=O^{\prime \prime}\left(|v|^{2}\right)$ in the sense of Section 1.3. To glue in the neck metric $g_{N}$ of Section 2.2, for instance, we would define $g_{t}=\beta_{1}\left(h+q^{\prime}\right)+\beta_{2}\left(1+t^{6(n-2)}|v|^{-(n-2)}\right)^{p-2} h$, where $\left(\beta_{1}, \beta_{2}\right)$ is 
the partition of unity defined in Section 2.1. Writing out the scalar curvature explicitly, we see that as $q^{\prime}=O^{\prime \prime}\left(|v|^{2}\right)$, the terms involving $q^{\prime}$ can be absorbed into the existing error terms in Section 2.4 so that (2.8) holds for the new metrics $g_{t}$. Therefore, Proposition 2.1 holds for the new metrics $g_{t}$ as well.

It can be seen by following the proofs of Proposition 2.2 and Theorem 3.9 in the appendix that no other nontrivial modifications are required to prove these results for the more general families of metrics $\left\{g_{t}: t \in(0, \delta)\right\}$ discussed above. Thus, the new metrics satisfy all the necessary conditions, and the results of Sections 3.2 and 3.3 apply to them without change.

4. Connected sums with zero scalar curvature. In this section, the methods of Sections 2 and 3 will be adapted to study zero scalar curvature manifolds. We have three cases to consider, when the scalar curvatures of $g^{\prime}$ and $g^{\prime \prime}$ are 0 and 1 , or 0 and 0 , or -1 and 0 . Each case introduces specific difficulties, and each needs some additional methods to prove the existence of constant scalar curvature metrics.

The first two cases fit into a common analytic framework, and will be handled together. The differences with the previous method are that $g_{t}$ must be defined more carefully than before to control the errors sufficiently, and in the analysis, the operator $a \Delta-v b$ now has one or two small eigenvalues. Thus, when the sequence $\left\{\phi_{i}\right\}_{i=0}^{\infty}$ is defined inductively using the inverse of this operator, the components in the directions of the corresponding eigenvectors have to be attended to, to prevent the sequence diverging. The third case is discussed in Section 4.5. We will outline what the constant scalar curvature metrics look like and how to prove existence results, but will not go into much detail.

4.1. Combining zero and positive scalar curvature. Let $\left(M^{\prime}, g^{\prime}\right)$ and $\left(M^{\prime \prime}, g^{\prime \prime}\right)$ be compact Riemannian $n$-manifolds such that $g^{\prime}$ has scalar curvature 0 and $g^{\prime \prime}$ scalar curvature 1 . Suppose, as in Section 2, that $M^{\prime}, M^{\prime \prime}$ contain points $m^{\prime}, m^{\prime \prime}$ with neighbourhoods in which $g^{\prime}, g^{\prime \prime}$ are conformally flat. As in Section 2.1, there exists a ball $B^{\prime}$ about $m^{\prime}$ in $M^{\prime}$ and a diffeomorphism $\Phi^{\prime}: B_{r}(0) \subset \mathbb{R}^{n} \rightarrow B^{\prime}$ for $r<1$ with $\Phi^{\prime}(0)=m^{\prime}$ and $\left(\Phi^{\prime}\right)^{*}\left(g^{\prime}\right)=\left(\psi^{\prime}\right)^{p-2} h$ for some function $\psi^{\prime}$ on $B_{r}(0)$ with $\psi^{\prime}(0)=1$ and $d \psi^{\prime}(0)=0$. As $g^{\prime}$ has zero scalar curvature, we have $\Delta \psi^{\prime}=0$ by (1.5).

Let $(\hat{M}, \hat{g})$ be the stereographic projection of $M^{\prime \prime}$ from $m^{\prime \prime}$. Then as in Section 2.1 , there is an immersion $\Xi^{\prime \prime}: \mathbb{R}^{n} \backslash \bar{B}_{R}(0) \rightarrow \hat{M}$ for some $R>0$, whose image is the complement of a compact set in $\hat{M}$, such that $\left(\Xi^{\prime \prime}\right)^{*}(\hat{g})=\xi^{p-2} h$ where $\xi$ is a smooth function on $\mathbb{R}^{n} \backslash \bar{B}_{R}(0)$ satisfying $\xi(v)=1+O^{\prime}\left(|v|^{2-n}\right)$. Also, by Proposition 1.3 , there exists $\mu \in \mathbb{R}$ such that

$$
\xi(v)=1+\mu|v|^{2-n}+O^{\prime}\left(|v|^{1-n}\right) .
$$

As $\left(M^{\prime \prime}, g^{\prime \prime}\right)$ is not conformal to $\mathscr{G}^{n}$ with its round metric, Theorem 1.4 shows that $\mu>0$, at least if $M^{\prime \prime}$ is spin or $n \leq 7$. For our purposes, we only 
need to assume that $\mu \neq 0$ so that $\mu|v|^{2-n}$ is the leading error term in (4.1). Therefore, if $n>7$ and $M^{\prime \prime}$ is not spin, we suppose that $\mu \neq 0$.

Choose $k$ with $(n-2)(n+2) / 2(n+1)<k<(n-2)(n+2) / 2 n$, which will remain fixed throughout this section. Choose $\delta \in(0,1)$ such that $\delta^{2 k /(n+2)} \leq r$ and $\delta^{-2 / n} \geq R$. We will write down a family of metrics $\left\{g_{t}: t \in(0, \delta)\right\}$ on $M=M^{\prime} \# M^{\prime \prime}$, in a similar way to Section 2.1. For any $t \in(0, \delta)$, define $M$ and the conformal class of $g_{t}$ by

$$
M=\left(M^{\prime} \backslash \Phi^{\prime}\left[\bar{B}_{t^{(n-2) / n}}(0)\right]\right) \amalg\left(\hat{M} \backslash\left(\Xi^{\prime \prime}\left[\mathbb{R}^{n} \backslash B_{t^{2 k /(n+2)-1}}(0)\right]\right)\right) /{ }_{t},
$$

where $\sim_{t}$ is the equivalence relation defined by

$$
\Phi^{\prime}[v] \sim{ }_{t} \Xi^{\prime \prime}\left[t^{-1} v\right] \quad \text { whenever } v \in \mathbb{R}^{n}, t^{(n-2) / n}<|v|<t^{2 k /(n+2)} .
$$

As in Section 2.1, the conformal class $\left[g_{t}\right]$ of $g_{t}$ is the restriction of the conformal classes of $g^{\prime}$ and $\hat{g}$ to the open sets of $M^{\prime}$ and $\hat{M}$ making up $M$, and is well defined because the conformal classes agree on the annulus of overlap $A_{t}$, where the two open sets are glued by $\sim_{t}$. Define $g_{t}$ within this conformal class by $g_{t}=g^{\prime}$ on the component of $M \backslash A_{t}$ coming from $M^{\prime}$, and $g_{t}=t^{2} \hat{g}$ on the component coming from $\hat{M}$. It remains to choose a conformal factor on $A_{t}$. This is done as in Section 2.1, except that the annulus $\left\{v \in \mathbb{R}^{n}: t^{(n-2) / n}<\right.$ $\left.|v|<t^{2 k /(n+2)}\right\}$ in $\mathbb{R}^{n}$ replaces $\left\{v \in \mathbb{R}^{n}: t^{2}<|v|<t\right\}$ in $\mathbb{R}^{n}$ in the definition of the partition of unity. This completes the definition of $g_{t}$ for $t \in(0, \delta)$.

LEMMA 4.1. Let the scalar curvature of $g_{t}$ be $-\epsilon_{t}$. Then $\epsilon_{t}$ is zero outside $A_{t}$. There exists $Y>0$ such that for all $t \in(0, \delta), \epsilon_{t}$ satisfies $\left|\epsilon_{t}\right| \leq Y$, and the volume of $A_{t}$ with respect to $g_{t}$ satisfies $\operatorname{vol}\left(A_{t}\right)=O\left(t^{2 n k /(n+2)}\right)$. Therefore, $g_{t}\left\|\epsilon_{t}\right\|_{L^{2 n /(n+2)}}=O\left(t^{k}\right)$ and ${ }^{g} t\left\|\epsilon_{t}\right\|_{L^{n / 2}}=O\left(t^{4 k /(n+2)}\right)$.

Proof. Outside $A_{t}$, the metric $g_{t}$ is equal to $g^{\prime}$ or homothetic to $\hat{g}$, and so has zero scalar curvature, verifying the first claim of the lemma. The proof that $\left|\epsilon_{t}\right| \leq Y$ is the same as that for the corresponding statement in Proposition 2.1, setting $v=0$. The estimate on the volume of $A_{t}$ also follows by the method used in Proposition 2.1, and the last two estimates are immediate.

We introduced $k$ above to make the estimate on ${ }^{g_{t}}\|\epsilon\|_{L^{2 n /(n+2)}}$ easy to write down.

LEMMA 4.2. For small $t$,

$$
\int_{M} \epsilon_{t} d V_{g_{t}}=(n-2) \omega_{n-1} \mu t^{n-2}+O\left(t^{n-2+\alpha}\right)
$$

where $\mu$ is the constant of (4.1), $\omega_{n-1}$ is the volume of the unit sphere $9^{n-1}$ in $\mathbb{R}^{n}$, and $\alpha=\min (2 / n, 2 k(n+1) /(n+2)-(n-2))>0$. 
Proof. Calculating with (1.5) gives

$$
\begin{aligned}
\epsilon_{t}(v)=\psi_{t}^{-(n+2) /(n-2)}(v)( & 2\left(\nabla \beta_{1}(v)\right) \cdot\left(\nabla\left(\psi^{\prime}(v)-\xi\left(t^{-1} v\right)\right)\right) \\
- & \left.\left(\Delta \beta_{1}(v)\right)\left(\psi^{\prime}(v)-\xi\left(t^{-1} v\right)\right)\right) .
\end{aligned}
$$

Let $F$ be the quadratic form on $\mathbb{R}^{n}$ given by the second derivatives of $\psi^{\prime}$; then $\psi^{\prime}=1+F+O^{\prime}\left(|v|^{3}\right)$. As the scalar curvature of $g^{\prime}$ is zero, the trace of $F$ is zero. Now $d V_{g_{t}}=\psi_{t}^{p} d V_{h}$. Multiplying (4.5) by this equation and making various estimates gives

$$
\begin{aligned}
\epsilon_{t}(v) d V_{g_{t}}=( & 2\left(\nabla \beta_{1}(v)\right) \cdot\left(\nabla F+O\left(|v|^{2}\right)-\mu t^{n-2} \nabla\left(|v|^{2-n}\right)-t^{n-1} O\left(|v|^{-n}\right)\right) \\
& \left.-\left(\Delta \beta_{1}(v)\right)\left(F+O\left(|v|^{3}\right)-\mu t^{n-2}|v|^{2-n}-t^{n-1} O\left(|v|^{1-n}\right)\right)\right) d V_{h}
\end{aligned}
$$

Integrate this over $A_{t}$. Now $\beta_{1}(v)=\beta(|v|)$ where $\beta(|v|)=\sigma(\log |v| / \log t)$ from Section 2.1. So $\left(\nabla \beta_{1}(v)\right) \cdot(\nabla F)=2|v|^{-1} F(d \beta / d x)$ and $\left(\nabla \beta_{1}(v)\right)$. $\left(-\mu t^{n-2} \nabla\left(|v|^{2-n}\right)\right)=(n-2) \mu t^{n-2}|v|^{1-n}(d \beta / d x)$ and $\Delta \beta_{1}=-\left(d^{2} \beta / d x^{2}\right)+$ $(1-n)|v|^{-1}(d \beta / d x)$. Therefore,

$$
\begin{aligned}
\int_{A_{t}} \epsilon_{t}(v) d V_{g_{t}} & \\
=\int_{A_{t}}( & \frac{d \beta}{d x}\left((n+3)|v|^{-1} F+(n-3) \mu t^{n-2}|v|^{1-n}+O\left(|v|^{2}\right)+t^{n-1} O\left(|v|^{-n}\right)\right) \\
& \left.\quad+|v| \frac{d^{2} \beta}{d x^{2}}\left(|v|^{-1} F-\mu t^{n-2}|v|^{1-n}+O\left(|v|^{3}\right)+t^{n-1} O\left(|v|^{1-n}\right)\right)\right) d V_{h}
\end{aligned}
$$

Using a Fubini theorem, we may write the integral on the right-hand side as a double integral over $\varphi^{n-1}$ and $|v|$. The volume forms are related by $d V_{h}=$ $|v|^{n-1} d \Omega d|v|$ where $d \Omega$ is the standard volume form on $\varphi^{n-1}$ with radius 1 . But as the trace of $F$ with respect to $h$ is zero, $\int_{g n-1} F d \Omega=0$ and the terms on the right-hand side of (4.7) involving $F$ vanish. So viewing (4.7) as a double integral and integrating over $\varphi^{n-1}$ gives

$$
\begin{aligned}
\int_{A_{t}} & \epsilon_{t}(v) d V_{g_{t}} \\
& =\omega_{n-1} \int_{t^{(n-2) / n}}^{t^{2 k /(n+2)}}\left((n-3) \mu t^{n-2} \frac{d \beta}{d x}-\mu t^{n-2} x \frac{d^{2} \beta}{d x^{2}}\right) d x+\text { error terms, }
\end{aligned}
$$

where $\omega_{n-1}$ is the volume of $\mathscr{9}^{n-1}$. 
The integral on the right-hand side is an exact integral, by parts. By definition, $\beta$ changes from 0 to 1 and $d \beta / d x$ from 0 to 0 over the interval, so, the integral becomes

$$
\int_{A_{t}} \epsilon_{t}(v) d V_{g_{t}}=(n-2) \omega_{n-1} \mu t^{n-2}+\text { error terms }
$$

which is nearly the conclusion of the lemma; it remains only to show that the "error terms" are of order $t^{n-2+\alpha}$.

This is a simple calculation and will be left to the reader, the necessary ingredients being that as $|v|$ lies between $t^{(n-2) / n}$ and $t^{2 k /(n+2)}, O(|v|)$ may be replaced by $O\left(t^{2 k /(n+2)}\right)$, and $t O\left(|v|^{-1}\right)$ may be replaced by $O\left(t^{2 / n}\right), d \beta / d x=$ $O\left(|v|^{-1}\right)$, and $d^{2} \beta / d x^{2}=O\left(|v|^{-2}\right)$. The error term that is usually the biggest is $O\left(|v|^{n+1}\right)$, and in order to ensure that this error term is smaller than the leading term calculated above, that is, to ensure $\alpha>0, k$ must satisfy $k>$ $(n+2)(n-2) / 2(n+1)$, which was one of the conditions in the definition of $k$ above.

The lemma shows that the average scalar curvature of $\left(M, g_{t}\right)$ is close to $-(n-2) \omega_{n-1} \mu t^{n-2} \operatorname{vol}\left(M^{\prime}\right)^{-1}$, so we will choose this value for the scalar curvature of $\tilde{g}_{t}$ in Section 4.4 .

4.2. Combining two metrics of zero scalar curvature. Let $M^{\prime}, M^{\prime \prime}, M, g^{\prime}, g^{\prime \prime}$, $m^{\prime}$, and $m^{\prime \prime}$ be as usual, with $g^{\prime}, g^{\prime \prime}$ of zero scalar curvature and conformally flat in neighbourhoods of $m^{\prime}, m^{\prime \prime}$. Rescaling $g^{\prime}, g^{\prime \prime}$ by homotheties still gives metrics of zero scalar curvature. Thus gluing these rescaled metrics, using the method of Section 2.2, gives a 2-parameter family of metrics in the same conformal class $\left[g_{t}\right]$ that all have small scalar curvature. Which of these metrics do we expect to be close to a metric of constant scalar curvature?

The necessary condition is that $\operatorname{vol}\left(M^{\prime}\right)=\operatorname{vol}\left(M^{\prime \prime}\right)$; we will explain why at the end of Section 4.3. Suppose, by applying a homothety to $g^{\prime}$ or $g^{\prime \prime}$ if necessary, that $\operatorname{vol}\left(M^{\prime}\right)=\operatorname{vol}\left(M^{\prime \prime}\right)$. A family of metrics $\left\{g_{t}: t \in(0, \delta)\right\}$ will be defined on $M$ following Section 4.1, such that when $t$ is small, $g_{t}$ resembles the union of $M^{\prime}$ and $M^{\prime \prime}$ with their metrics $\mathcal{g}^{\prime}$ and $\mathcal{g}^{\prime \prime}$, joined by a small neck of approximate radius $t$, which is modelled upon the manifold $N$ of Section 2.2, with metric $t^{2} g_{N}$.

Choose $k$ with $(n-2)(n+2) / 2(n+1)<k<(n-2)(n+2) / 2 n$ and apply the gluing method of Section 4.1 twice, once to glue one asymptotically flat end of $\left(N, t^{2} g_{N}\right)$ into $M^{\prime}$ at $m^{\prime}$, and once to glue the other asymptotically flat end into $M^{\prime \prime}$ at $m^{\prime \prime}$. The role of $A_{t}$ in Section 4.1 is played by $A_{t}=A_{t}^{\prime} \cup A_{t}^{\prime \prime}$, the disjoint union of annuli $A_{t}^{\prime}$ joining $N$ and $M^{\prime}$, and $A_{t}^{\prime \prime}$ joining $N$ and $M^{\prime \prime}$. With this definition, we state the next two lemmas, which are analogues of Lemmas 4.1 and 4.2 . 
LEMMA 4.3. Let the scalar curvature of $g_{t}$ be $-\epsilon_{t}$. Then $\epsilon_{t}$ is zero outside $A_{t}$. There exists $Y>0$ such that for all $t \in(0, \delta), \epsilon_{t}$ satisfies $\left|\epsilon_{t}\right| \leq Y$, and the volume of $A_{t}$ with respect to $g_{t}$ satisfies $\operatorname{vol}\left(A_{t}\right)=O\left(t^{2 n k /(n+2)}\right)$. Therefore, $g_{t}\left\|\epsilon_{t}\right\|_{L^{2 n /(n+2)}}=O\left(t^{k}\right)$ and ${ }^{g} t\left\|\epsilon_{t}\right\|_{L^{n / 2}}=O\left(t^{4 k /(n+2)}\right)$.

Proof. This is identical to Lemma 4.1, and its proof is the same except that $g_{t}$ may also be homothetic to $g_{N}$ in the first sentence.

LEMMA 4.4. For all small $t$,

$$
\begin{aligned}
& \int_{A_{t}^{\prime}} \epsilon_{t} d V_{g_{t}}=(n-2) \omega_{n-1} t^{n-2}+O\left(t^{n-2+\alpha}\right), \\
& \int_{A_{t}^{\prime \prime}} \epsilon_{t} d V_{g_{t}}=(n-2) \omega_{n-1} t^{n-2}+O\left(t^{n-2+\alpha}\right),
\end{aligned}
$$

where $\alpha=\min (2 / n, 2 k(n+1) /(n+2)-(n-2))>0$ and $\omega_{n-1}$ is the volume of the unit sphere $\mathscr{S}^{n-1}$ in $\mathbb{R}^{n}$.

Proof. This is merely Lemma 4.2 applied twice, firstly to the gluing of $N$ into $M^{\prime}$ and secondly to the gluing of $N$ into $M^{\prime \prime}$. We have also used the observation that for both asymptotically flat ends of $N$, the constant $\mu$ of Section 4.1 takes the value 1 . To see this, compare the definition of $\mu$ in Proposition 1.3 with the definition of $\left(N, g_{N}\right)$ in Section 2.2.

4.3. Inequalities on the connected sum. Now we derive the analytic inequalities needed for the main result in Section 4.4. First of all, observe that Proposition 2.2 applies to the metrics of Sections 4.1 and 4.2.

LEMMA 4.5. Let $\left\{g_{t}: t \in(0, \delta)\right\}$ be one of the families of metrics defined on $M=M^{\prime} \# M^{\prime \prime}$ in Sections 4.1 and 4.2. Then there exist $A>0$ and $\zeta \in(0, \delta)$ such that ${ }^{g} t\|\phi\|_{L^{p}} \leq A \cdot g_{t}\|\phi\|_{L_{1}^{2}}$ whenever $\phi \in L_{1}^{2}(M)$ and $t \in(0, \zeta]$.

Proof. The proof follows that of Proposition 2.2, applied to the metrics of Sections 4.1 and 4.2 rather than Sections 2.1 and 2.2, except for some simple changes to take into account, the different powers of $t$ used to define the new metrics.

As in Section 3.3, to calculate with the inverse of $a \Delta-v b$, we need to know about the spectrum of $a \Delta$ on $\left(M, g_{t}\right)$. The next three results give the necessary information; the proofs are again deferred to the appendix.

THEOREM 4.6. Let $\left\{g_{t}: t \in(0, \delta)\right\}$ be the family of metrics defined on $M=$ $M^{\prime} \# M^{\prime \prime}$ in Section 4.1. Choose $\gamma>0$ such that a $\Delta$ on $\left(M^{\prime}, g^{\prime}\right)$ has no eigenvalues in $(0,2 \gamma)$. Then $a \Delta$ on $\left(M, g_{t}\right)$ has no eigenvalues in $(0, \gamma)$ for small $t$.

For the metrics $g_{t}$ of Section 4.2, the situation is more complicated. For small $t$, we expect the eigenvectors of $a \Delta$ on $\left(M, g_{t}\right)$ with small eigenvalues to 
be close to eigenvectors of $a \Delta$ on $M^{\prime}$ or $M^{\prime \prime}$ with small eigenvalues, that is, to constant functions on $M^{\prime}$ and $M^{\prime \prime}$. So we expect two eigenvectors on $\left(M, g_{t}\right)$ associated to small eigenvalues, one is the constant function, and the other close to a constant on the $M^{\prime}$ part of $M$, and to a different constant on the $M^{\prime \prime}$ part of $M$. The next result describes this second eigenvector. The proof is deferred to the appendix.

Proposition 4.7. Let $\left\{g_{t}: t \in(0, \delta)\right\}$ be the family of metrics defined on $M=M^{\prime} \# M^{\prime \prime}$ in Section 4.2. Then for small $t$, there exists $\lambda_{t}>0$ and $\beta_{t} \in C^{\infty}(M)$ such that $a \Delta \beta_{t}=\lambda_{t} \beta_{t}$ on $\left(M, g_{t}\right)$. Here $\lambda_{t}=O\left(t^{n-2}\right)$, and

$$
\beta_{t}= \begin{cases}1+O\left(t^{n-2}\right) & \text { on } M^{\prime} \backslash B^{\prime}, \\ 1+O\left(t^{n-2}|v|^{2-n}\right) & \text { on }\{v: t \leq|v|<\delta\} \subset B^{\prime}, \\ -1+O\left(t^{n-2}\right) & \text { on } M^{\prime \prime} \backslash B^{\prime \prime}, \\ -1+O\left(t^{n-2}|v|^{2-n}\right) & \text { on }\{v: t \leq|v|<\delta\} \subset B^{\prime \prime},\end{cases}
$$

identifying subsets of $M^{\prime}, M^{\prime \prime}$ with subsets of $M$.

The proposition is proved by a series method, starting with a function that is 1 on the part of $M$ coming from $M^{\prime}$ and -1 on the part coming from $M^{\prime \prime}$, and then adding small corrections to get to an eigenvector of $a \Delta$. Note that $\beta_{t}$ takes the approximate values \pm 1 on the two halves because $\operatorname{vol}\left(M^{\prime}\right)=\operatorname{vol}\left(M^{\prime \prime}\right)$ by assumption; if the volumes were different, then the approximate values would have to be adjusted so that $\int_{M} \beta_{t} d V_{g_{t}}=0$.

We may now state the analogue of Theorem 4.6 for the metrics of Section 4.2, which will be proved in the appendix.

THEOREM 4.8. Let $\left\{g_{t}: t \in(0, \delta)\right\}$ be the family of metrics defined on $M=$ $M^{\prime} \# M^{\prime \prime}$ in Section 4.2. Choose $\gamma>0$ such that all as has no eigenvalues in $(0,2 \gamma)$ on $\left(M^{\prime}, g^{\prime}\right)$ or $\left(M^{\prime \prime}, g^{\prime \prime}\right)$. Then for small $t$, the only eigenvalue of $a \Delta$ in $(0, \gamma)$ on $\left(M, g_{t}\right)$ is $\lambda_{t}$ from Proposition 4.7 , with eigenspace $\left\langle\beta_{t}\right\rangle$.

Theorems 4.6 and 4.8 will fit into the existence proofs of Section 4.4 in the same way as Theorem 3.9 does into that of Section 3.3. The small eigenvalue $\lambda_{t}$ in Theorem 4.8 means that $\beta_{t}$-components of functions will have to be carefully controlled, to ensure that inverting $a \Delta$ upon them does not give a large result. We now bound the $\beta_{t}$-component of $\epsilon_{t}$.

LEMMA 4.9. Let $\left\{g_{t}: t \in(0, \delta)\right\}$ be the family of metrics defined on $M=$ $M^{\prime} \# M^{\prime \prime}$ in Section 4.2. Then for small $t$,

$$
\int_{M} \beta_{t} \epsilon_{t} d V_{g_{t}}=O\left(t^{n-2+\alpha}\right)
$$

where $\beta_{t}$ is the function of Proposition 4.7 and $\alpha$ is as in Lemma 4.4. 
Proof. Proposition 4.7 shows that $\beta_{t}=1+O\left(t^{2(n-2) / n}\right)$ on $A_{t}^{\prime}$ and $\beta_{t}=$ $-1+O\left(t^{2(n-2) / n}\right)$ on $A_{t}^{\prime \prime}$, as these are annuli in which $t^{(n-2) / n}<|v|<t^{2 k /(n+2)}$. Applying these and Lemmas 4.3 and 4.4 to the integral of $\beta_{t} \epsilon_{t}$ over $M$ gives

$$
\int_{M} \beta_{t} \epsilon_{t} d V_{g_{t}}=O\left(t^{n-2+\alpha}\right)+Y \operatorname{vol}\left(A_{t}\right) \cdot O\left(t^{2(n-2) / n}\right),
$$

and as $\operatorname{vol}\left(A_{t}\right)=O\left(t^{2 n k /(n+2)}\right)$, the second term is $O\left(t^{2 n k /(n+2)+2(n-2) / n}\right)$. But by the definitions of $k$ and $\alpha$, it is easily shown that $n-2+\alpha<2 n k /(n+2)+$ $2(n-2) / n$, and so the first error term is larger and subsumes the second, as required.

We note that this lemma is the reason for requiring that $\operatorname{vol}\left(M^{\prime}\right)=\operatorname{vol}\left(M^{\prime \prime}\right)$. For if the two are not equal, then Lemma 4.4 still shows that $\int_{A_{t}^{\prime}} \epsilon_{t} d V_{g_{t}}$ and $\int_{A_{t}^{\prime \prime}} \epsilon_{t} d V_{g_{t}}$ are equal to highest order, but $\beta_{t}$ takes values approximately proportional to $\operatorname{vol}\left(M^{\prime}\right)^{-1}$ on $A_{t}^{\prime}$, and to $\operatorname{vol}\left(M^{\prime \prime}\right)^{-1}$ on $A_{t}^{\prime \prime}$. Thus in this case, $\int_{M} \beta_{t} \epsilon_{t} d V_{g_{t}}$ is $O\left(t^{n-2}\right)$ rather than $O\left(t^{n-2+\alpha}\right)$. But we will need $\int_{M} \beta_{t} \epsilon_{t} d V_{g_{t}}=$ $o\left(t^{n-2}\right)$ for the proof in Section 4.4.

4.4. Existence of constant scalar curvature metrics. Now we give the existence results for constant scalar curvature metrics on the connected sums of Sections 4.1 and 4.2 .

THEOREM 4.10. Let $\left\{g_{t}: t \in(0, \delta)\right\}$ be one of the families of metrics on $M=M^{\prime} \# M^{\prime \prime}$ defined in Sections 4.1 and 4.2. Then there exists $C>0$ such that $g_{t}$ admits a smooth conformal rescaling for small $t$ to $\tilde{g}_{t}=(1+\phi)^{p-2} g_{t}$, with scalar curvature $-(n-2) \omega_{n-1} \mu t^{n-2} \operatorname{vol}\left(M^{\prime}\right)^{-1}$ in Section 4.1 and $-(n-$ 2) $\omega_{n-1} t^{n-2} \operatorname{vol}\left(M^{\prime}\right)^{-1}$ in Section 4.2, and ${ }^{g}\|\phi\|_{L_{1}^{2}} \leq C t^{\alpha}$. Here $\omega_{n-1}$ is the volume of the unit sphere $\varphi^{n-1}$ in $\mathbb{R}^{n}$, and $\mu, \alpha$ are as in Section 4.1, and we suppose $\mu \neq 0$ in the cases not covered by Theorem 1.4.

Proof. Let $D_{0}$ be $(n-2) \omega_{n-1} \mu \operatorname{vol}\left(M^{\prime}\right)^{-1}$ in Section 4.1 and $(n-2) \omega_{n-1} \times$ $\operatorname{vol}\left(M^{\prime}\right)^{-1}$ in Section 4.2. Define a function $\eta$ on $\left(M, g_{t}\right)$ by $\eta=\epsilon_{t}-D_{0} t^{n-2}$. Then $g_{t}$ has scalar curvature $-D_{0} t^{n-2}-\eta$. As in Section 3, the condition for $\tilde{g}_{t}=(1+\rho+\tau)^{p-2} g_{t}$ to have scalar curvature $-D_{0} t^{n-2}$ is

$$
a \Delta(\rho+\tau)+b D_{0} t^{n-2}(\rho+\tau)=\eta+\eta \cdot(\rho+\tau)-D_{0} t^{n-2} f(\rho+\tau) .
$$

Define a vector space $P$ of functions on $\left(M, g_{t}\right)$ by $P=\langle 1\rangle$ in Section 4.1, and $P=\left\langle 1, \beta_{t}\right\rangle$ in Section 4.2, where $\beta_{t}$ is as in Proposition 4.7. We will construct $\rho, \tau$ satisfying (4.14), with $\rho \in P$ and $\tau \in P^{\perp}$ with respect to the $L_{1}^{2}$ inner product.

Define inductively sequences $\left\{\rho_{i}\right\}_{i=0}^{\infty}$ of elements of $P$ and $\left\{\tau_{i}\right\}_{i=0}^{\infty}$ of elements of $P^{\perp} \subset L_{1}^{2}(M)$ by $\rho_{0}=\tau_{0}=0$, and having defined the sequences up to 
$i-1$, let $\rho_{i}$ and $\tau_{i}$ be the unique elements of $P$ and $P^{\perp}$ satisfying

$$
\begin{aligned}
& a \Delta\left(\rho_{i}+\tau_{i}\right)+b D_{0} t^{n-2}\left(\rho_{i}+\tau_{i}\right) \\
& \quad=\eta+\eta \cdot\left(\rho_{i-1}+\tau_{i-1}\right)-D_{0} t^{n-2} f\left(\rho_{i-1}+\tau_{i-1}\right) .
\end{aligned}
$$

If we can show that these sequences converge to $\rho \in P$ and $\tau \in P^{\perp}$ that are small when $t$ is small, then the arguments of Section 3 complete the theorem.

The difficulty lies in inverting the operator $a \Delta+b D_{0} t^{n-2}$ : by Theorems 4.6 and 4.8, the operator is invertible on $P^{\perp}$ with inverse bounded by $\gamma^{-1}$, as all the eigenvectors of $a \Delta$ in $P^{\perp}$ have eigenvalues at least $\gamma$. But on $P$, the inverse is of order $t^{2-n}$, which is large; so $\rho_{i}$ may be large even if the right-hand side of (4.15) is small.

The solution is to ensure that the $P$ components of $\eta$ are smaller even than $t^{n-2}$ so that, after applying the inverse of $a \Delta+b D_{0} t^{n-2}$ to them, they are still small. Let $\pi$ denote orthogonal projection onto $P$; both the $L^{2}$ and the $L_{1}^{2}$ inner product give the same answer, and in fact, the projection makes sense even in $L^{1}(M)$. Then from (4.15), we make the estimates

$$
\begin{aligned}
&\left\|\rho_{i}\right\|_{L_{1}^{2}} \leq D_{1} t^{2-n}\left(\|\pi(\eta)\|_{L^{1}}+\left\|\pi\left(\eta \rho_{i-1}\right)\right\|_{L^{1}}+\left\|\pi\left(\eta \tau_{i-1}\right)\right\|_{L^{1}}\right) \\
&+D_{2}\left\|\pi\left(f\left(\rho_{i-1}+\tau_{i-1}\right)\right)\right\|_{L^{1}} \\
&\left\|\tau_{i}\right\|_{L_{1}^{2}} \leq D_{3}\left(\|\eta\|_{L^{2 n /(n+2)}}+\left\|\eta \rho_{i-1}\right\|_{L^{2 n /(n+2)}}+\left\|\eta \tau_{i-1}\right\|_{L^{2 n /(n+2)}}\right. \\
&\left.\quad+D_{0} t^{n-2}\left\|f\left(\rho_{i-1}+\tau_{i-1}\right)\right\|_{L^{2 n /(n+2)}}\right)
\end{aligned}
$$

for some constants $D_{1}, D_{2}$, and $D_{3}$ independent of $t$. The norms on the righthand side of (4.16) would normally be $L^{2 n /(n+2)}$ norms, but as $P$ is a finitedimensional space, all norms are equivalent, and we may use the $L^{1}$ norm.

Our strategy is to show that if $\left\|\rho_{i-1}\right\|_{L_{1}^{2}} \leq D_{4} t^{\alpha}$ and $\left\|\tau_{i-1}\right\|_{L_{1}^{2}} \leq D_{5} t^{k}$ for large enough constants $D_{4}, D_{5}$, then $\left\|\rho_{i}\right\|_{L_{1}^{2}} \leq D_{4} t^{\alpha}$ and $\left\|\tau_{i}\right\|_{L_{1}^{2}} \leq D_{5} t^{k}$ also hold for small $t$, so by induction, the sequences are bounded; convergence for small $t$ easily follows by a similar argument to that used in Lemma 3.2.

From Lemmas 4.2, 4.4, and 4.9, we deduce that $\|\pi(\eta)\|_{L^{1}}=O\left(t^{n-2+\alpha}\right)$, so the first term on the right of (4.16) contributes $O\left(t^{\alpha}\right)$ to $\left\|\rho_{i}\right\|_{L_{1}^{2}}$, consistent with $\left\|\rho_{i}\right\|_{L_{1}^{2}} \leq D_{4} t^{\alpha}$ if $D_{4}$ is chosen large enough. The third term $\left\|\pi\left(\eta \tau_{i-1}\right)\right\|_{L^{1}}$ is bounded by $A\|\eta\|_{L^{2 n /(n+2)}}\left\|\tau_{i-1}\right\|_{L_{1}^{2}}$, and $\|\eta\|_{L^{2 n /(n+2)}}=O\left(t^{k}\right)$ by Lemmas 4.1 and 4.3; the third term therefore contributes $O\left(t^{2 k+2-n}\right)$ to $\left\|\rho_{i}\right\|_{L_{1}^{2}}$, and by the definition of $\alpha$, this error term is strictly smaller than $O\left(t^{\alpha}\right)$. The fourth error term is also easily shown to be smaller than $O\left(t^{\alpha}\right)$.

Thus, the second term in (4.16) is the only problem, the reason is that the $P^{\perp}$-component of $\eta$, multiplied by $\rho_{i-1}$, may have an appreciable component in $P$. We get round this as follows. Suppose $\xi \in P^{\perp}$ and $\rho \in P$, and consider the $P$-component of $\xi \rho$. In Section 4.1, this component is zero, and there is no problem; in Section 4.2, there may be a component in the direction of $\beta_{t}$, and 
it is measured by $\int_{M} \xi \beta_{t}^{2} d V_{g_{t}}$. But by the description of $\beta_{t}$ in Proposition 4.7, $\beta_{t}^{2}$ is close to 1 and $\xi$ is orthogonal to the constants, and so in general the $P$-component of $\xi \rho$ will be small compared to the sizes of $\xi$ and $\rho$. Taking this into account, it is easy to get a good bound on $\left\|\pi\left(\eta \rho_{i-1}\right)\right\|_{L^{1}}$.

The rest of the proof will be left to the reader. What remains to be done is to prove inductively that the bounds $\left\|\rho_{i}\right\|_{L_{1}^{2}} \leq D_{4} t^{\alpha},\left\|\tau_{i}\right\|_{L_{1}^{2}} \leq D_{5} t^{k}$ hold for small enough $t$, and then, to prove the convergence of the sequences, and these may both be done using the methods of Lemma 3.2, working from (4.16) and (4.17). Setting $\phi=\rho+\tau$, where $\rho, \tau$ are the limits of the sequences. The reader may then rejoin the proof of Theorem 3.1 after Lemma 3.3.

As the metrics constructed have negative scalar curvature, they are unique in their conformal classes, and are Yamabe metrics. The theorem thus tells us that the Yamabe metric on the connected sum, with small neck of two zero scalar curvature manifolds, balances the volumes of the two component manifolds so that they are equal, a fact which seems rather appealing.

4.5. Combining zero and negative scalar curvature. There is just one case left, that of gluing a metric of zero scalar curvature into a metric of scalar curvature -1 . This case can be handled using the results of Section 3 and using the following simple extension of the method: we define a family of metrics $\left\{g_{t}: t \in(0, \delta)\right\}$ on the connected sum $M$, with $-1-\epsilon_{t}$ the scalar curvature of $g_{t}$, and then prove that $\left|\epsilon_{t}\right| \leq Y,\left\|\epsilon_{t}\right\|_{L^{n / 2}} \leq Z t^{l}$ as in Proposition 2.1, and that $\|\phi\|_{L^{p}} \leq A t^{-\kappa}\|\phi\|_{L_{1}^{2}}$ for $\phi \in L_{1}^{2}(M)$ as in Proposition 2.2, where norms are taken with respect to $g_{t}$.

Here $\iota, \kappa>0$. The idea is that if $\iota$ is large compared to $\kappa$, then we may follow the proofs of Section 3 adding in powers of $t$, and at the crucial stages when we need some expression to be sufficiently small, the power of $t$ will turn out to be positive, and so we only need to take $t$ small enough. To do this in practice, we modify the proof slightly to cut down the number of applications of Proposition 2.2, and thus obtain a more favourable necessary ratio of $\iota$ to $\mathrm{K}$.

Now $\kappa$ is essentially determined by what the Yamabe metric on the connected sum actually looks like-if $t$ is the radius of the neck, any family of good approximations to the Yamabe metrics will have $\kappa=(n-2) / n$ (this value will be justified below). So, the problem is to make $\iota$ large enough, in other words, to start with a family of metrics $g_{t}$ that are a good approximation to scalar curvature -1 . We will not go through the construction and proof again for this case, but will describe how to define metrics $g_{t}$ that have close enough to constant scalar curvature for the method outlined above to work.

Consider the connected sum $M$ of $\left(M^{\prime}, g^{\prime}\right)$ with scalar curvature -1 and $\left(M^{\prime \prime}, g^{\prime \prime}\right)$ with scalar curvature 0 . To get a good enough approximation to scalar curvature -1 , we have to rescale $g^{\prime \prime}$ so that its scalar curvature approximates -1 rather than 0 . Let $\xi$ be the Green's function of $a \Delta$ at $m^{\prime \prime}$ on $M^{\prime \prime}$ satisfying $a \Delta \xi=\delta_{m^{\prime \prime}}-\operatorname{vol}\left(M^{\prime \prime}\right)^{-1}$ in the sense of distributions. Since $\xi$ is only defined 
up to the addition of a constant, choose $\xi$ to have minimum value 0 . Then $\xi$ is a $C^{\infty}$-function on $\hat{M}=M^{\prime \prime} \backslash\left\{m^{\prime \prime}\right\}$ with a pole at $m^{\prime \prime}$, of the form $(n-$ 2) $\omega_{n-1}^{-1}|v|^{2-n}+O^{\prime}\left(|v|^{1-n}\right)$, in the usual coordinates.

Let $\hat{g}_{t}=\left(t^{(n-2)^{2} / 2 n}+t^{(n-2)(n+2) / 2 n} \operatorname{vol}\left(M^{\prime \prime}\right) \xi\right)^{p-2} g^{\prime \prime}$ on $\hat{M}$. Calculating its scalar curvature $\hat{S}_{t}$ using (1.5) gives

$$
\hat{S}_{t}=-\left(1+t^{2(n-2) / n} \operatorname{vol}\left(M^{\prime \prime}\right) \xi\right)^{-(n+2) /(n-2)}
$$

so that $-1 \leq \hat{S}_{t}<0$, and $\hat{S}_{t}$ is close to -1 away from $m^{\prime \prime}$ for small $t$. Outside a small neighbourhood of $m^{\prime \prime}, \hat{g}_{t}$ is close to $t^{2(n-2) / n} g^{\prime \prime}$ so that the diameter of $M^{\prime \prime}$ is multiplied by $t^{(n-2) / n}$. But in a small neighbourhood of $m^{\prime \prime}, \hat{g}_{t}$ resembles a neck metric of radius proportional to $t$, as in Section 2.2. So $\hat{g}_{t}$ looks like $M^{\prime \prime}$ rescaled by $t^{(n-2) / n}$, and with a neck of radius proportional to $t$, opening out to an asymptotically flat end.

We construct $g_{t}$ by gluing $\hat{g}_{t}$ into $g^{\prime}$ using the natural neck. Thus, a rough description of $g_{t}$ is that it is $g^{\prime}$ on the $M^{\prime}$ part and $t^{2(n-2) / n} \mathcal{g}^{\prime \prime}$ on the $M^{\prime \prime}$ part, and the two parts are joined by a neck with radius proportional to $t$. With a family $\left\{g_{t}: t \in(0, \delta)\right\}$ of metrics defined in this way, the modified method outlined above may be applied to show that there exist small conformal deformations of $g_{t}$ to scalar curvature -1 , for $t$ sufficiently small.

One final point: we can now see the reason for the failure of Proposition 2.2 which necessitated this whole detour. Consider a smooth function $\phi$ on $\left(M, g_{t}\right)$ that is 0 on the $M^{\prime}$ part, 1 on the $M^{\prime \prime}$ part, and changes only on the neck. A simple calculation shows that for such a function $\|\phi\|_{L^{p}} \sim t^{(2-n) / n}\|\phi\|_{L_{1}^{2}}$, and so the value for $\kappa$ given above is the least possible.

4.6. Doing without conformal flatness. In Section 3.4, we explained that the results of Section 3 still hold if the assumption, that $g^{\prime}, g^{\prime \prime}$ are conformally flat in neighbourhoods of $m^{\prime}, m^{\prime \prime}$, is dropped. However, the results of Section 4 do require modifications to generalize in this way. The problem is in extending Lemmas 4.2 and 4.4 to the curved case: we need a quite precise evaluation of the total scalar curvature of $g_{t}$, and have to be careful that the error terms do not swamp the term that we can evaluate.

To deal with the case of Section 4.1 first, it can be shown that the proof of Lemma 4.2 still holds when $g^{\prime}$ has conformal curvature near $m^{\prime}$, because defining $g_{t}$ as in Section 3.4, the extra error terms introduced in the scalar curvature can be absorbed into the error terms already in (4.6), and so the proof of Lemma 4.2 holds from that point. But if we allow $g^{\prime \prime}$ to be conformally curved near $m^{\prime \prime}$, then Proposition 1.3 does not hold, and the scalar curvature of $g_{t}$ is dominated (except for $n=3,4,5$ ) by error terms that seem to have no nice expression.

Therefore, the situation is this. Theorem 4.10 applies without change when the metrics $g_{t}$ of Section 4.1 are defined using $g^{\prime \prime}$ conformally flat near $m^{\prime \prime}$, but $g^{\prime}$ not necessarily conformally flat near $m^{\prime}$. To include the case $g^{\prime \prime}$ not 
conformally flat, the result will hold if we weaken it so as not to prescribe the constant value that the scalar curvature takes, but merely give an estimate of its magnitude. Also, we believe that the result applies as stated when $n=3,4$, or 5 because then the mass term is large enough to dominate the errors.

The case of Section 4.2 is easier; Theorem 4.10 applies without change to the metrics $g_{t}$ of Section 4.2 defined using $g^{\prime}, g^{\prime \prime}$ not supposed conformally flat about $m^{\prime}$ and $m^{\prime \prime}$. This is because the metrics $g_{t}$ are made by gluing in the neck metric $t^{2} g_{N}$, which is conformally flat, so it reduces to the case of Section 4.1 when $g^{\prime \prime}$ is locally conformally flat, which we have already seen works.

This leaves the case of Section 4.5. The generalization of Proposition 2.2 suggested there will extend without change to the conformally curved case, so the problem is to define the metrics $g_{t}$ in such a way that the extra error terms, introduced in the expression for the scalar curvature, still give good enough approximations to constant scalar curvature for the existence result to apply. We think that this can be done quite readily just by working on how to produce good approximations $g_{t}$, say, by adding a well-chosen conformal factor to the existing definition.

\section{Appendix}

The spectrum of $a \Delta$ on $M^{\prime} \# M^{\prime \prime}$. In this appendix, we prove Theorems 3.9, 4.6, and 4.8, and Proposition 4.7. They are results on the eigenvalues and eigenvectors of the operator $a \Delta$ on $M$ with the metrics $g_{t}$, defined in Sections 3 and 4. They appear here and not in the main text because the proofs are long calculations.

Theorem 3.9 takes up Sections A.1 and A.2. Its proof divides naturally into considering eigenvalues of $a \Delta$ smaller than $b$ and eigenvalues larger than $b$. The eigenvectors with eigenvalues smaller than $b$ form a finite dimensional space $E$. In Section A.1, we define a vector space $E_{t}$ that is a good approximation to $E$ when $t$ is small, and using this, we show that all eigenvalues of vectors in $E$ are at most $b-\gamma$. In Section A.2, we consider eigenvectors with eigenvalues larger than $b$, which must therefore be orthogonal to $E$, and by considering their inner product with $E_{t}$, we can show that their eigenvalues must be at least $b+\gamma$.

In Section A.3, we prove similar results for use in the zero scalar curvature material of Section 4. Most of the work needed to prove them has already been done in Sections A.1 and A.2, and the main problem is the construction of an eigenvector $\beta_{t}$ of $a \Delta$ with a small eigenvalue $\lambda_{t}$. This is done by a sequence method, the basic idea being to start with an approximation to $\beta_{t}$ and repeatedly invert $a \Delta$ upon it; as $\lambda_{t}$ is the smallest positive eigenvalue, the $\beta_{t^{-}}$ component of the resulting sequence grows much faster than any other and so comes to dominate. 
A.1. Eigenvalues of $a \Delta$ smaller than $b$. We now prove Theorem 3.9.

Proof OF THeOrem 3.9. It is well known that the spectrum of the Laplacian on a compact Riemannian manifold is discrete and nonnegative, and that the eigenspaces are finite dimensional. Therefore, on $M^{\prime}$ and $M^{\prime \prime}$, there are only finitely many eigenvalues of $a \Delta$ smaller than $b$, and to each is associated a finite-dimensional space of eigenfunctions.

Let $E^{\prime}$ be the finite-dimensional vector space of smooth functions on $M^{\prime}$ generated by eigenfunctions of $a \Delta$ on $M^{\prime}$ associated with eigenvalues less than $b$; we think of $E^{\prime}$ as a subspace of $L_{1}^{2}\left(M^{\prime}\right)$. For Section 2.2, define $E^{\prime \prime}$ on $M^{\prime \prime}$ in the same way. As $a \Delta$ has no eigenvalues in the interval $(b-2 \gamma, b+2 \gamma)$, we see that

$$
\begin{aligned}
& \text { if } \phi \in E^{\prime}, \quad \text { then } \int_{M^{\prime}} a|\nabla \phi|^{2} d V_{g^{\prime}} \leq \int_{M^{\prime}}(b-2 \gamma) \phi^{2} d V_{g^{\prime}}, \\
& \text { if } \phi \in\left(E^{\prime}\right)^{\perp} \subset L_{1}^{2}\left(M^{\prime}\right), \quad \text { then } \int_{M^{\prime}} a|\nabla \phi|^{2} d V_{g^{\prime}} \geq \int_{M^{\prime}}(b+2 \gamma) \phi^{2} d V_{g^{\prime}},
\end{aligned}
$$

and also two analogous inequalities for $M^{\prime \prime}$ in Section 2.2. The perpendicular subspace $\left(E^{\prime}\right)^{\perp}$ of (A.2) may be taken with respect to the inner product of $L_{1}^{2}\left(M^{\prime}\right)$ or with respect to that of $L^{2}\left(M^{\prime}\right)$ - both give the same space as $E^{\prime}$ is a sum of eigenspaces of $a \Delta$.

Now if we have two statements like (A.1) and (A.2) but applying to $M$ rather than $M^{\prime}$, then we can prove the result. This is the content of the next lemma.

LEMMA A.11. Suppose there is a subspace $E_{t}$ of $L_{1}^{2}(M)$ for small $t$ such that

$$
\begin{aligned}
& \text { if } \phi \in E_{t}, \text { then } \int_{M} a|\nabla \phi|^{2} d V_{g_{t}} \leq \int_{M}(b-\gamma) \phi^{2} d V_{g_{t}}, \\
& \text { if } \phi \in\left(E_{t}\right)^{\perp} \subset L_{1}^{2}(M) \text {, then } \int_{M} a|\nabla \phi|^{2} d V_{g_{t}} \geq \int_{M}(b+\gamma) \phi^{2} d V_{g_{t}} \text {, }
\end{aligned}
$$

defining $\left(E_{t}\right)^{\perp}$ with the $L_{1}^{2}$ inner product. Then Theorem 3.9 holds.

Proof. Suppose $E_{t}$ exists. We must show that $l \in(b-\gamma, b+\gamma)$ cannot be an eigenvalue of $a \Delta$ on $\left(M, g_{t}\right)$. Suppose $\phi$ is an eigenfunction of $a \Delta$ with this eigenvalue $l$. Let $\phi_{1}$ and $\phi_{2}$ be the components of $\phi$ in $E_{t}$ and $\left(E_{t}\right)^{\perp}$, respectively. Then, as $a \Delta \phi-l \phi=0$,

$$
\begin{aligned}
0 & =\int_{M}\left(\phi_{2}-\phi_{1}\right)\left(a \Delta\left(\phi_{1}+\phi_{2}\right)-l\left(\phi_{1}+\phi_{2}\right)\right) d V_{g_{t}} \\
& =\int_{M}\left(a\left|\nabla \phi_{2}\right|^{2}-l \phi_{2}^{2}\right) d V_{g_{t}}-\int_{M}\left(a\left|\nabla \phi_{1}\right|^{2}-l \phi_{1}^{2}\right) d V_{g_{t}} \\
& \geq \int_{M}\left((\gamma+l-b) \phi_{1}^{2}+(\gamma+b-l) \phi_{2}^{2}\right) d V_{g_{t}}
\end{aligned}
$$

using (A.3) and (A.4) in the last line. But as $\gamma+l-b, \gamma+b-l>0$, this shows that $\phi_{1}=\phi_{2}=\phi=0$, which is a contradiction. 
To complete the proof of the theorem, we therefore need to produce some spaces $E_{t}$ of functions on $M$ satisfying (A.3) and (A.4). In Section 2.1, $E_{t}$ should be modelled on $E^{\prime}$, and in Section 2.2, on $E^{\prime} \oplus E^{\prime \prime}$.

As a half-way stage between $E^{\prime}, E^{\prime \prime}$, and $E_{t}$, spaces $\tilde{E}^{\prime}, \tilde{E}^{\prime \prime}$ of functions on $M^{\prime}$, $M^{\prime \prime}$ will be made, that are close to $E^{\prime}, E^{\prime \prime}$ but vanish on small balls around $m^{\prime}$, $m^{\prime \prime}$. Let $\sigma^{\prime}$ be a $C^{\infty}$-function on $M^{\prime}$ that is 1 on the complement of a small ball about $m^{\prime}, 0$ on a smaller ball about $m^{\prime}$, and otherwise, taking values in $[0,1]$. Now define $\tilde{E}^{\prime}=\sigma^{\prime} E^{\prime}=\left\{\sigma^{\prime} v: v \in E^{\prime}\right\}$.

By choosing the ball outside which $\sigma^{\prime}$ is 1 to be small, we can ensure that $\tilde{E}^{\prime}$ is close to $E^{\prime}$ in $L_{1}^{2}\left(M^{\prime}\right)$ in the following sense: the two have the same dimension, and any $\tilde{v} \in \tilde{E}^{\prime}$ may be written as $\tilde{v}=v_{1}+v_{2}$, where $v_{1}, v_{2} \in E^{\prime},\left(E^{\prime}\right)^{\perp}$, respectively, and satisfy

$$
\left\|v_{2}\right\|_{L_{1}^{2}}^{2} \leq \frac{\gamma}{2(a+b+2 \gamma)}\left\|v_{1}\right\|_{L_{1}^{2}}^{2}
$$

Suppose that $\sigma^{\prime}$ has been chosen so that these hold. Then two statements similar to (A.1) and (A.2) hold for $\tilde{E}^{\prime}$, as we will see in the next lemma.

LEMMA A.12. The subspace $\tilde{E}^{\prime}$ satisfies the following two conditions:

$$
\begin{aligned}
& \text { if } \phi \in \tilde{E}^{\prime}, \text { then } \int_{M^{\prime}} a|\nabla \phi| d V_{g^{\prime}} \leq \int_{M^{\prime}}\left(b-\frac{3}{2} \gamma\right) \phi^{2} d V_{g^{\prime}}, \\
& \text { if } \phi \in\left(\tilde{E}^{\prime}\right)^{\perp} \subset L_{1}^{2}\left(M^{\prime}\right), \text { then } \int_{M^{\prime}} a|\nabla \phi|^{2} d V_{g^{\prime}} \geq \int_{M^{\prime}}\left(b+\frac{3}{2} \gamma\right) \phi^{2} d V_{g^{\prime}},
\end{aligned}
$$

where the inner product used to construct $\left(\tilde{E}^{\prime}\right)^{\perp}$ is that of $L_{1}^{2}\left(M^{\prime}\right)$.

Proof. First we prove (A.7). Let $\phi \in \tilde{E}^{\prime}$, then $\phi=v_{1}+v_{2}$ with $v_{1} \in E^{\prime}$ and $v_{2} \in\left(E^{\prime}\right)^{\perp}$. Because $v_{1}$ and $v_{2}$ are orthogonal in both $L^{2}$ and $L_{1}^{2}$,

$$
\begin{aligned}
a \int_{M^{\prime}} & |\nabla \phi|^{2} d V_{g^{\prime}} \\
\quad & a \int_{M^{\prime}}\left|\nabla v_{1}\right|^{2} d V_{g^{\prime}}+a \int_{M^{\prime}}\left|\nabla v_{2}\right|^{2} d V_{g^{\prime}} \\
& \leq a \int_{M^{\prime}}\left|\nabla v_{1}\right|^{2} d V_{g^{\prime}}+a|| v_{2} \|_{L_{1}^{2}}^{2} \\
& \leq a \int_{M^{\prime}}\left|\nabla v_{1}\right|^{2} d V_{g^{\prime}}+\frac{a \gamma}{2(a+b+2 \gamma)}|| v_{1} \|_{L_{1}^{2}}^{2} \\
& =a\left(1+\frac{\gamma}{2(a+b+2 \gamma)}\right) \int_{M^{\prime}}\left|\nabla v_{1}\right|^{2} d V_{g^{\prime}}+\frac{a \gamma}{2(a+b+2 \gamma)} \int_{M^{\prime}} v_{1}^{2} d V_{g^{\prime}} \\
& \leq\left((b-2 \gamma)\left(1+\frac{\gamma}{2(a+b+2 \gamma)}\right)+\frac{a \gamma}{2(a+b+2 \gamma)}\right) \int_{M^{\prime}} v_{1}^{2} d V_{g^{\prime}} \\
& \leq\left(b-\frac{3}{2} \gamma\right) \int_{M^{\prime}} \phi^{2} d V_{g^{\prime}} \cdot
\end{aligned}
$$


Here between the third and fourth lines we have used (A.6), between the fifth and sixth lines we have used (A.1), and between the last two we have used the $L^{2}$-orthogonality of $v_{1}, v_{2}$, and the trivial inequality $(b-2 \gamma)[1+\gamma / 2(a+b+$ $2 \gamma)]+a \gamma / 2(a+b+2 \gamma) \leq b-3 \gamma / 2$. This proves (A.7).

To prove (A.8), observe that by (A.6), orthogonal projection from $\tilde{E}^{\prime}$ to $E^{\prime}$ is injective, and as they have the same (finite) dimension, it must also be surjective. Let $\tilde{v}_{2} \in\left(\tilde{E}^{\prime}\right)^{\perp}$. Then $\tilde{v}_{2}=v_{1}+v_{2}$ with $v_{1} \in E^{\prime}$ and $v_{2} \in\left(E^{\prime}\right)^{\perp}$. By this surjectivity, there exists $\tilde{v}_{1} \in \tilde{E}^{\prime}$ such that $\tilde{v}_{1}=v_{1}+v_{3}$ with $v_{3} \in\left(E^{\prime}\right)^{\perp}$, that is, the $E^{\prime}$-component of $\tilde{v}_{1}$ is $v_{1}$, the same as that of $\tilde{v}_{2}$. But $\tilde{v}_{1}$ and $\tilde{v}_{2}$ are orthogonal in $L_{1}^{2}\left(M^{\prime}\right)$, so taking their inner product gives that $\left\|v_{1}\right\|_{L_{1}^{2}}^{2}=-\left\langle v_{2}, v_{3}\right\rangle \leq$ $\left\|v_{3}\right\|_{L_{1}^{2}}\left\|v_{2}\right\|_{L_{1}^{2}}$.

As $\tilde{v}_{1}=v_{1}+v_{3} \in \tilde{E}^{\prime}$, we may square this inequality, substitute in for $\left\|v_{3}\right\|_{L_{1}^{2}}^{2}$ using (A.6), and divide through by $\left\|v_{1}\right\|_{L_{1}^{2}}^{2}$. The result is that

$$
\left\|v_{1}\right\|_{L_{1}^{2}}^{2} \leq \frac{\gamma}{2(a+b+2 \gamma)}\left\|v_{2}\right\|_{L_{1}^{2}}^{2}, \quad \text { for } \tilde{v}_{2}=v_{1}+v_{2} \in\left(\tilde{E}^{\prime}\right)^{\perp}
$$

which is the analogue of (A.6) for $\left(\tilde{E}^{\prime}\right)^{\perp}$ instead of $\tilde{E}^{\prime}$. This is the ingredient needed to prove (A.8) by the method used above for (A.7), and the remainder of the proof will be left to the reader.

For the case of Section 2.2, a subspace $\tilde{E}^{\prime \prime}$ of functions on $M^{\prime \prime}$ is created in the same way, and Lemma A.12 applies to this space too. We now define the spaces $E_{t}$. In Section 2.1, let $E_{t}$ be the space of functions that are equal to some function in $\tilde{E}^{\prime}$ on the subset of $M$ identified with $M^{\prime} \backslash \Phi^{\prime}\left[\bar{B}_{t}(0)\right]$, and are zero outside this subset. In Section 2.2, let $E_{t}$ be the direct sum of this space of functions, and the corresponding space made from $\tilde{E}^{\prime \prime}$.

For small $t$, the functions in $E_{t}$ are $C^{\infty}$, and on their support, $g_{t}$ is equal to $g^{\prime}$ (or $g^{\prime \prime}$ in case of Section 2.2). Thus, (A.7) applies to functions in $E_{t}$.

LEMMA A.13. For small enough $t$, the subspace $E_{t}$ of $L_{1}^{2}(M)$ satisfies

$$
\text { if } \phi \in E_{t}, \quad \text { then } \int_{M} a|\nabla \phi|^{2} d V_{g_{t}} \leq \int_{M}\left(b-\frac{3}{2} \gamma\right) \phi^{2} d V_{g_{t}} \text {. }
$$

A fortiori, it satisfies the inequality (A.3) of Lemma A.11.

Proof. In Section 2.1, this follows immediately from (A.7) as the $g_{t}$ and $g^{\prime}$ agree upon the support of the functions of $E_{t}$. In Section 2.2, $\phi$ is the sum of elements of $\tilde{E}^{\prime}$ and $\tilde{E}^{\prime \prime}$; both sides of (A.11) split into two terms, each involving one function. So (A.11) is the sum of two inequalities, which follow immediately from (A.7) as before, and from the counterpart of (A.7) applying to $\tilde{E}^{\prime \prime}$. 
The previous lemma showed that the space of functions $E_{t}$ upon $M$ satisfies inequality (A.3) of Lemma A.11. In the next proposition, proved in Section A.2, we show that the inequality (A.4) is satisfied too.

Proposition A.14. Let $M, g_{t}$, and $E_{t}$ be as in Section A.1. Then for small $t$, inequality (A.4) of Lemma A.11 holds.

The proof of this proposition is the subject of Section A.2. Suppose, for the moment, that the proposition holds. Then a space of functions $E_{t}$ upon $M$ has been constructed, satisfying inequality (A.3) by Lemma A.13, and inequality (A.4) by Proposition A.14. So by Lemma A.11, the proof of Theorem 3.9 is finished.

A.2. Eigenvalues of $a \Delta$ larger than $b$. Next we will prove Proposition A.14 which completes the proof of Theorem 3.9. The idea of the proof is as follows. Given $\phi \in\left(E_{t}\right)^{\perp}$, we want to show that its average eigenvalue of $a \Delta$ is at least $b+\gamma$. We do this in different ways depending on whether $\phi$ is concentrated in $M^{\prime}$, or $M^{\prime \prime}$, or the neck in between.

Proof of Proposition A.14. For simplicity, we will prove the proposition for the metrics of Section 2.1 only, and the modifications for Section 2.2 will be left to the reader. We will start from (A.8) of Lemma A.12. The constants in (A.4) and (A.8) are different-the first has $b+\gamma$ and the second $b+3 \gamma / 2$. Choose constants $b_{0}>b_{1}>\cdots>b_{5}$ with $b_{0}=b+3 \gamma / 2$ and $b_{5}=b+\gamma$. These will be used to contain five error terms.

Shortly, we will choose $r_{1}, r_{2}, r_{3}$ with $0<r_{1}<r_{2}<r_{3}$. Define three compact Riemannian submanifolds with boundary $R_{t} \subset S_{t} \subset T_{t}$ in $\left(M, g_{t}\right)$ to be the subsets of $M$ coming from subsets $R, S$, and $T$ of $M^{\prime \prime}$, respectively, where

$$
\begin{gathered}
R=\hat{M} \backslash \Xi^{\prime \prime}\left[\mathbb{R}^{n} \backslash \bar{B}_{r_{1}}(0)\right], \quad S=\hat{M} \backslash \Xi^{\prime \prime}\left[\mathbb{R}^{n} \backslash \bar{B}_{r_{2}}(0)\right], \\
T=\hat{M} \backslash \Xi^{\prime \prime}\left[\mathbb{R}^{n} \backslash \bar{B}_{r_{3}}(0)\right] .
\end{gathered}
$$

When $t$ is small, $R_{t}, S_{t}$, and $T_{t}$ lie in the region of $M$ in which the function $\beta_{2}$, used in Section 2.1 to define $g_{t}$, is 1 . Then $R_{t}, S_{t}$, and $T_{t}$ are homothetic to $R, S$, and $T$, respectively, by a homothety multiplying their metrics by $t^{12}$.

The idea is this. A diffeomorphism $\Psi_{t}^{\prime}$ from $M^{\prime} \backslash\left\{m^{\prime}\right\}$ onto $M \backslash R_{t}$ will be constructed, which will be the identity outside $T_{t}$. Using $\Psi_{t}^{\prime}$, any function in $L_{1}^{2}(M)$ defines a function in $L_{1}^{2}\left(M^{\prime}\right)$. Applying (A.8) of Lemma A.12 therefore induces an inequality upon functions in $L_{1}^{2}(M)$. We will be able to show that for functions that are not too large in $S_{t}$, this inequality implies (A.4) as we require. Then only the case of functions that are large in $S_{t}$ remains.

Suppose, for the moment, that $r_{1}, r_{2}$, and $r_{3}$ are fixed with $r_{1}<r_{2}<r_{3}$. For $R_{t}$ to be well defined, $r_{1}$ must satisfy $r_{1}>\delta^{-4}$. For $T_{t}$ to be well defined, $t$ must be sufficiently small that $t^{6} r_{3}<\delta$. We also suppose that $t$ is small enough that the functions of $E_{t}$ vanish on $T_{t}$. 
Let $\Psi_{t}^{\prime}: M^{\prime} \backslash\left\{m^{\prime}\right\} \rightarrow M$ be the identity outside $\Phi^{\prime}\left[B_{t^{6} r_{3}}(0)\right]$ in $M^{\prime}$, and on $\Phi^{\prime}\left[B_{t^{6} r_{3}}(0)\right]$ in $M^{\prime}$ define $\Psi_{t}^{\prime}$ by

$$
\Psi_{t}^{\prime}\left(\Phi^{\prime}(v)\right)=\Phi^{\prime}\left(\frac{t^{6} r_{1} v}{|v|}+\frac{\left(r_{3}-r_{1}\right) v}{r_{3}}\right)
$$

Let $\phi \in L_{1}^{2}(M)$ and define $\phi^{\prime}=\left(\Psi_{t}^{\prime}\right)^{*}(\phi)$. Then $\phi^{\prime} \in L_{1}^{2}\left(M^{\prime}\right)$, as we will see.

An easy calculation shows that

$$
b_{0} \int_{M^{\prime}}\left(\phi^{\prime}\right)^{2} d V_{g^{\prime}}=b_{0} \int_{M} \phi^{2} \cdot F_{t} d V_{g_{t}},
$$

where $F_{t}$ is a function on $M$ that is 1 on that part of $M$ coming from $M^{\prime} \backslash$ $\Phi^{\prime}\left[B_{t^{6} r_{3}}(0)\right]$, is zero on that part of $M$ not coming from $M^{\prime} \backslash \Phi^{\prime}\left[B_{t}{ }^{6} r_{1}(0)\right]$, and in the intermediate annulus is given by

$$
F_{t}\left(\left(\phi^{\prime}\right)^{-1}(v)\right)=\frac{\left(|v|-t^{6} r_{1}\right)^{n-1} r_{3}^{n}}{|v|^{n-1}\left(r_{3}-r_{1}\right)^{n}} \cdot \psi^{\prime}(v)^{p} \psi_{t}(v)^{-p}
$$

Similarly, we may easily show that

$$
a \int_{M^{\prime}}\left|\nabla \phi^{\prime}\right|^{2} d V_{g^{\prime}} \leq a \int_{M}|\nabla \phi|^{2} \cdot G_{t} d V_{g_{t}},
$$

where $G_{t}$ is a function on $M$ that is 1 on that part of $M$ coming from $M^{\prime} \backslash$ $\Phi^{\prime}\left[B_{t}{ }^{6} r_{3}(0)\right]$, is zero on that part of $M$ not coming from $M^{\prime} \backslash \Phi^{\prime}\left[B_{t}{ }^{6} r_{1}(0)\right]$, and in the intermediate annulus is given by

$$
\begin{aligned}
& G_{t}\left(\left(\phi^{\prime}\right)^{-1}(v)\right) \\
& \quad=\max \left(\frac{\left(|v|-t^{6} r_{1}\right)^{n-1} r_{3}^{n+2}}{|v|^{n-1}\left(r_{3}-r_{1}\right)^{n+2}}, \frac{\left(|v|-t^{6} r_{1}\right)^{n-3} r_{3}^{n-2}}{|v|^{n-3}\left(r_{3}-r_{1}\right)^{n-2}}\right) \cdot \psi^{\prime}(v) \psi_{t}(v)^{-1} .
\end{aligned}
$$

Here, the first term in the $\max (\cdots)$ is the multiplier for the radial component of $\nabla \phi$, and the second term is the multiplier for the nonradial components. As $F_{t}, G_{t}$ are bounded, we see from (A.14) and (A.16) that $\phi^{\prime} \in L_{1}^{2}\left(M^{\prime}\right)$, as was stated above.

Suppose now that $\phi \in\left(E_{t}\right)^{\perp} \subset L_{1}^{2}(M)$. For small enough $t$, this implies that $\phi^{\prime} \in\left(\tilde{E}^{\prime}\right)^{\perp}$, and so (A.8) applies by Lemma A.12. Combining this with (A.14) and (A.16) gives

$$
a \int_{M}|\nabla \phi|^{2} \cdot G_{t} d V_{g_{t}} \geq b_{0} \int_{M} \phi^{2} \cdot F_{t} d V_{g_{t}}
$$

Now by the definition of $\psi_{t}, \psi^{\prime}(v) \psi_{t}(v)^{-1}$ approaches 1 as $t \rightarrow 0$. In fact, it may be shown that

$$
\left|\psi^{\prime}(v) \psi_{t}(v)^{-1}-1\right| \leq C_{0} t^{6(n-2)}|v|^{-(n-2)} \quad \text { when } t^{6} \leq|v| \leq t^{6-2 /(n-2)},
$$


for some constant $C_{0}$. For $t$ small enough, this certainly holds in the region $t^{6} r_{1} \leq|v| \leq t^{6} r_{3}$, and in this region, we have $\left|\psi^{\prime}(v) \psi_{t}(v)^{-1}-1\right| \leq C_{0} r_{1}^{-(n-2)}$.

Choose $r_{1}$ greater than $\delta^{-4}$, and large enough that $b_{1}\left(1+C_{0} r_{1}^{-(n-2)}\right)^{p} \leq b_{0}$ and $b_{2} \leq b_{1}\left(1-C_{0} r_{1}^{-(n-2)}\right)$. Then for small $t$, the $\psi^{\prime} \psi_{t}^{-1}$ terms in $F_{t}$ and $G_{t}$ can be absorbed by putting $b_{2}$ in place of $b_{0}$. Next, $r_{2}$ is defined uniquely in terms of $r_{3}$ to satisfy $r_{1}<r_{2}<r_{3}$ and $b_{3}\left(r_{2}-r_{1}\right)^{n-1} r_{3}^{n} r_{2}^{1-n}\left(r_{3}-r_{1}\right)^{-n}=b_{4}$. Then $b_{3}\left(|v|-t^{6} r_{1}\right)^{n-1} r_{3}^{n}|v|^{1-n}\left(r_{3}-r_{1}\right)^{-n} \geq b_{4}$ when $t^{6} r_{2} \leq|v| \leq t^{6} r_{3}$. This is to bound $F_{t}$ below on the region $|v| \geq t^{6} r_{2}$.

Finally, we define $r_{3}$. Choose $r_{3}>r_{1}$ sufficiently large that two conditions hold. The first is that $b_{3} \cdot \max \left(\left(|v|-t^{6} r_{1}\right)^{n-1} r_{3}^{n+2}|v|^{1-n}\left(r_{3}-r_{1}\right)^{-(n+2)},(|v|-\right.$ $\left.\left.t^{6} r_{1}\right)^{n-3} r_{3}^{n-2}|v|^{3-n}\left(r_{3}-r_{1}\right)^{2-n}\right) \leq b_{2}$ when $t^{6} r_{1} \leq|v| \leq t^{6} r_{3}$; combining this with one of the inequalities used to define $r_{1}$ shows that $b_{3} G_{t} \leq b_{1}$. The second condition is that

$$
\frac{\operatorname{vol}(S)}{\operatorname{vol}(T)-\operatorname{vol}(S)} \leq \frac{b_{4}-b_{5}}{4 b_{5}}
$$

This condition will be needed later.

The last two definitions are circular as $r_{2}$ is defined in terms of $r_{3}$, and vice versa because $S_{t}$ depends on $r_{2}$. However, manipulating the definition of $r_{2}$ reveals that, however large $r_{3}$ is, $r_{2}$ must satisfy $r_{2} \leq r_{1}\left(1-b_{3}^{1 /(n-1)} b_{2}^{-1 /(n-1)}\right)^{-1}$, and so $\operatorname{vol}(S)$ is bounded in terms of $r_{1}$, whereas $\operatorname{vol}(T)$ can grow arbitrarily large. Therefore, (A.20) does hold for $r_{3}$ sufficiently large.

The above estimates show that $G_{t} \leq b_{1} / b_{3}$ and $F_{t} \geq b_{1} b_{4} / b_{0} b_{3}$ on $M \backslash S_{t}$ for small $t$. Substituting these into (A.18) gives that, when $t$ is sufficiently small,

$$
a \int_{M}|\nabla \phi|^{2} d V_{g_{t}} \geq b_{4} \int_{M \backslash S_{t}} \phi^{2} d V_{g_{t}}
$$

Suppose that $\int_{S_{t}} \phi^{2} d V_{g_{t}} \leq\left(b_{4} / b_{5}-1\right) \cdot \int_{M \backslash S_{t}} \phi^{2} d V_{g_{t}}$. Then $b_{4} \int_{M \backslash S_{t}} \phi^{2} d V_{g_{t}} \geq$ $b_{5} \int_{M} \phi^{2} d V_{g_{t}}$, and from (A.21), we see that (A.4) holds for $\phi$, which is what we have to prove. Therefore, it remains only to deal with the case that $\int_{S_{t}} \phi^{2} d V_{g_{t}}>$ $\left(b_{4} / b_{5}-1\right) \cdot \int_{M \backslash S_{t}} \phi^{2} d V_{g_{t}}$.

Suppose that this inequality holds. The basic idea of the remainder of the proof is that when $t$ is small, the volume of $S_{t}$ is also small, and this forces $\phi$ to be large on $S_{t}$ compared to its average value elsewhere. Therefore, $\phi$ must change substantially in the neighbourhood $T_{t}$ of $S_{t}$, and this forces $\nabla \phi$ to be large in $T_{t}$.

Restrict $t$ further, to be small enough that $t^{6} r_{3} \leq t^{2}$. Then $T_{t}$ is contained in the region of gluing in which $\beta_{2}=1$. So the pair $\left(S_{t}, T_{t}\right)$ is homothetic to a pair $(S, T)$ of compact manifolds with $C^{\infty}$ boundaries and with $S$ contained in the interior of $T$; the metrics on $\left(S_{t}, T_{t}\right)$ are the metrics on $(S, T)$ multiplied by $t^{12}$. For these $S, T$, the following lemma holds. 
LEMMA A.15. Let $S, T$ be compact, connected Riemannian $n$-manifolds with smooth boundaries such that $S \subset T$ but $S \neq T$. Then there exists $C_{1}>0$ such that for all $\phi \in L_{1}^{2}(T)$,

$$
\left(\frac{\int_{S} \phi^{2} d V_{g}}{\operatorname{vol}(S)}\right)^{1 / 2}-\left(\frac{\int_{T \backslash S} \phi^{2} d V_{g}}{\operatorname{vol}(T)-\operatorname{vol}(S)}\right)^{1 / 2} \leq C_{1}\left(\int_{T}|\nabla \phi|^{2} d V_{g}\right)^{1 / 2} .
$$

Proof. We begin by quoting a theorem on the existence of solutions of the equation $\Delta u=f$ on a manifold with smooth boundary.

THEOREM A.16. Suppose that $T$ is a compact manifold with smooth boundary, and that $f \in L^{2}(T)$ and satisfies $\int_{T} f d V_{g}=0$. Then there exists $\xi \in L_{2}^{2}(T)$, unique up to the addition of a constant such that $\Delta \xi=f$, and in addition, $\mathbf{n} \cdot \nabla \xi$ vanishes at the boundary, where $\mathbf{n}$ is the unit outward normal to the boundary.

Proof. This is a partial statement of [4, Example 2, page 65]. Hörmander's example is only stated for $C^{\infty}$-functions $f$ and $\xi$, but the proof works for $f \in$ $\mathscr{H}_{(0)}(T)$ and $\xi \in \mathscr{H}_{(2)}(T)$ in his notation, which are $L^{2}(T)$ and $L_{2}^{2}(T)$ in ours. $\square$

Put $f=\operatorname{vol}(S)^{-1}$ in $S$ and $f=(\operatorname{vol}(S)-\operatorname{vol}(T))^{-1}$ in $T \backslash S$. Then $\int_{T} f d V_{g}=0$, so by the theorem, there exists a function $\xi \in L_{2}^{2}(M)$ satisfying $\Delta \xi=f$, and that $\nabla \xi$ vanishes normal to the boundary. Because of this vanishing, the boundary term has dropped out of the following integration-by-parts equation:

$$
\int_{T} \phi \Delta \xi=-\int_{T}(\nabla \phi) \cdot(\nabla \xi) d V_{g}
$$

Substituting in for $\Delta \xi$ and using Hölder inequality gives

$$
\begin{aligned}
\frac{1}{\operatorname{vol}(S)}\left|\int_{S} \phi d V_{g}\right| & -\frac{1}{\operatorname{vol}(T)-\operatorname{vol}(S)}\left|\int_{T \backslash S} \phi d V_{g}\right| \\
& \leq\left(\int_{T}|\nabla \xi|^{2} d V_{g}\right)^{1 / 2} \cdot\left(\int_{T}|\nabla \phi|^{2} d V_{g}\right)^{1 / 2} .
\end{aligned}
$$

Now $S$ is connected, so the constants are the only eigenvectors of $\Delta$ on $S$ with eigenvalue 0 and derivative vanishing normal to the boundary. By the discreteness of the spectrum of $\Delta$ on $S$ with these boundary conditions, there is a positive constant $K_{S}$ less than or equal to all the positive eigenvalues. It easily follows that for $\phi \in L_{1}^{2}(S)$,

$$
\left(\frac{\int_{S} \phi^{2} d V_{g}}{\operatorname{vol}(S)}\right)^{1 / 2} \leq \frac{1}{\operatorname{vol}(S)}\left|\int_{S} \phi d V_{g}\right|+\left(\frac{\int_{S}|\nabla \phi|^{2} d V_{g}}{K_{S} \cdot \operatorname{vol}(S)}\right)^{1 / 2} .
$$

Also, a simple application of Hölder inequality yields

$$
\frac{1}{\operatorname{vol}(T)-\operatorname{vol}(S)}\left|\int_{T \backslash S} \phi d V_{g}\right| \leq\left(\frac{\int_{T \backslash S} \phi^{2} d V_{g}}{\operatorname{vol}(T)-\operatorname{vol}(S)}\right)^{1 / 2} .
$$


Adding together (A.24), (A.25), and (A.26) gives (A.22), as we want, with constant $C_{1}=\left(\int_{T}|\nabla \xi|^{2} d V_{g}\right)^{1 / 2}+\left(K_{S} \cdot \operatorname{vol}(S)\right)^{-1 / 2}$.

The point of this calculation is that because $\left(S_{t}, T_{t}\right)$ are homothetic to $(S, T)$ by the constant factor $t^{12}$, Lemma A.15 implies that for all $\phi \in L_{1}^{2}\left(T_{t}\right)$,

$$
\left(\frac{\int_{S_{t}} \phi^{2} d V_{g_{t}}}{\operatorname{vol}(S)}\right)^{1 / 2}-\left(\frac{\int_{T_{t} \backslash S_{t}} \phi^{2} d V_{g_{t}}}{\operatorname{vol}(T)-\operatorname{vol}(S)}\right)^{1 / 2} \leq C_{1} t^{6}\left(\int_{T_{t}}|\nabla \phi|^{2} d V_{g_{t}}\right)^{1 / 2}
$$

Now, using (A.20), it follows that

$$
\begin{aligned}
\left(\int_{S_{t}} \phi^{2} d V_{g_{t}}\right)^{1 / 2} & -\left(\frac{b_{4}-b_{5}}{4 b_{5}} \int_{M \backslash S_{t}} \phi^{2} d V_{g_{t}}\right)^{1 / 2} \\
& \leq \operatorname{vol}(S)^{1 / 2} C_{1} t^{6}\left(\int_{M}|\nabla \phi|^{2} d V_{g_{t}}\right)^{1 / 2}
\end{aligned}
$$

But because $\int_{S_{t}} \phi^{2} d V_{g_{t}}>\left(b_{4} / b_{5}-1\right) \cdot \int_{M \backslash S_{t}} \phi^{2} d V_{g_{t}}$, substituting this into (A.28), squaring, and manipulating gives that

$$
b_{5} \int_{M} \phi^{2} d V_{g_{t}}<\frac{b_{4} b_{5}}{b_{4}-b_{5}} \cdot \int_{S_{t}} \phi^{2} d V_{g_{t}}<\frac{4 b_{4} b_{5} t^{12} C_{1}^{2} \operatorname{vol}(S)}{b_{4}-b_{5}} \cdot \int_{M}|\nabla \phi|^{2} d V_{g_{t}} .
$$

Therefore, if $t$ is small, then inequality (A.4) holds. This completes the proof of Proposition A.14.

A.3. The spectrum of $a \Delta$ in the zero scalar curvature cases. Now we prove Proposition 4.7 and Theorems 4.6 and 4.8. We will start with a preliminary version of Theorems 4.6 and 4.8. Suppose $\gamma>0$ such that $a \Delta$ has no eigenvalues in $(0,2 \gamma)$ on $\left(M^{\prime}, g^{\prime}\right)$ in Section 4.1 , and on both $\left(M^{\prime}, g^{\prime}\right)$ and $\left(M^{\prime \prime}, g^{\prime \prime}\right)$ in Section 4.2. Then

if $\phi \in L_{1}^{2}\left(M^{\prime}\right), \int_{M^{\prime}} \phi d V_{g^{\prime}}=0$, then $\int_{M^{\prime}} a|\nabla \phi|^{2} d V_{g^{\prime}} \geq \int_{M^{\prime}} 2 \gamma \phi^{2} d V_{g^{\prime}}$, (A.30)

and the same for $M^{\prime \prime}$ in Section 4.2. This is an analogue of (A.2) of Section A.1, and the analogues of $E^{\prime}$ and $E^{\prime \prime}$ are the spaces of constant functions on $M^{\prime}$ and $M^{\prime \prime}$.

Define spaces of functions $E_{t}$ on $M$ as in Section A.1. This involves choosing a function $\sigma^{\prime}$ on $M^{\prime}$ (done just before Lemma A.12) that is 1 outside a small ball around $m^{\prime}$, and a similar function $\sigma^{\prime \prime}$ on $M^{\prime \prime} . B^{\prime}, B^{\prime \prime}$.For the proof of 
Proposition 4.7 later, we must choose $\sigma^{\prime}, \sigma^{\prime \prime}$ to vary with $t$ rather than being fixed as they were before. Let $\sigma^{\prime}, \sigma^{\prime \prime}$ be functions on $M^{\prime}, M^{\prime \prime}$ defined in the same way as $\beta_{1}, \beta_{2}$ in Section 4.1 , but which are 0 when $|v| \leq t^{2 k /(n+2)}$ and 1 when $|v| \geq t^{(n-2) /(n+1)}$ and outside

This makes sense for small $t$, as the definition of $k$ in Section 4.1 gives $2 k /(n+2)>(n-2) /(n+1)$ so that $t^{2 k /(n+2)}<t^{(n-2) /(n+1)}<\delta$ for small $t$. Note also that the choice of the inner radius $t^{2 k /(n+2)}$ means (from Section 4.1) that $g_{t}$ is identified with $g^{\prime}$ and $g^{\prime \prime}$ on the support of $\sigma^{\prime}$ and $\sigma^{\prime \prime}$, respectively, so that the functions $E_{t}$ are supported in the parts of $M$ where $g_{t}$ equals $g^{\prime}$ or $g^{\prime \prime}$. Here is a first approximation to Theorems 4.6 and 4.8:

LEMMA A.17. Let $\left\{g_{t}: t \in(0, \delta)\right\}$ be one of the families of metrics defined in Sections 4.1 and 4.2. Then for small $t$,

$$
\text { if } \phi \in\left(E_{t}\right)^{\perp} \subset L_{1}^{2}(M), \text { then } \int_{M} a|\nabla \phi|^{2} d V_{g_{t}} \geq \gamma \int_{M} \phi^{2} d V_{g_{t}} \text {. }
$$

Here the orthogonal space is taken with respect to the $L^{2}$ inner product.

Proof. This is proved just as is Proposition A.14, except that we use the inner product in $L^{2}(M)$ rather than that in $L_{1}^{2}(M)$, and instead of choosing a series of constants interpolating between $b+2 \gamma$ and $b+\gamma$, we choose constants interpolating between $2 \gamma$ and $\gamma$, and some simple changes must be made to the proof as the powers of $t$, used in defining the metrics of Sections 4.1 and 4.2, are different to those used in Sections 2.1 and 2.2.

Now as $E_{t}$ is modelled on $E^{\prime}$ in Section 4.1 and on $E^{\prime} \oplus E^{\prime \prime}$ in Section 4.2, it is close to the constant functions in Section 4.1, and to functions taking one constant value on the $M^{\prime}$ part of $M$ and another constant value on the $M^{\prime \prime}$ part in Section 4.2.

These spaces $E_{t}$ are not quite good enough for our purposes, for we will need spaces that contain the constants. We therefore produce modified spaces $\tilde{E}_{t}$, and prove a similar lemma for them. In Section 4.1, let $\tilde{E}_{t}=\langle 1\rangle$. In Section 4.2, let $e \in E_{t}$ be the unique element that is nonnegative on the part of $M$ coming from $M^{\prime}$ and satisfies $\int_{M} e d V_{g_{t}}=0$ and $\int_{M} e^{2} d V_{g_{t}}=2 \operatorname{vol}\left(M^{\prime}\right)$, and let $\tilde{E}_{t}=$ $\langle 1, e\rangle$.

LEMMA A.18. Let $\left\{g_{t}: t \in(0, \delta)\right\}$ be one of the families of metrics defined in Sections 4.1 and 4.2. Then for small $t$,

$$
\text { if } \phi \in\left(\tilde{E}_{t}\right)^{\perp} \subset L_{1}^{2}(M), \text { then } \int_{M} a|\nabla \phi|^{2} d V_{g_{t}} \geq \gamma \int_{M} \phi^{2} d V_{g_{t}} \text {. }
$$

Here the orthogonal space is taken with respect to the $L^{2}$ inner product.

Proof. Let $\xi$ be the unique element of $E_{t}$ with $\int_{M} \xi d V_{g_{t}}=1$, and $\int_{M} e \xi d V_{g_{t}}=$ 0 in Section 4.2. If $\phi \in\left(\tilde{E}_{t}\right)^{\perp}$, then $\phi-\langle\phi, \xi\rangle \in E_{t}^{\perp}$ where $\langle$,$\rangle is the inner product$ 
of $L^{2}(M)$. So, by Lemma A.17,

$$
\begin{aligned}
\int_{M} a|\nabla \phi|^{2} d V_{g_{t}} & \geq \gamma \int_{M}(\phi-\langle\phi, \xi\rangle)^{2} d V_{g_{t}} \\
& =\gamma \int_{M}\left(\phi^{2}+\langle\phi, \xi\rangle^{2}\right) d V_{g_{t}} \\
& \geq \gamma \int_{M} \phi^{2} d V_{g_{t}} .
\end{aligned}
$$

Here between the first and second lines, we have used the fact that $\phi \in\left(\tilde{E}_{t}\right)^{\perp}$, and thus $\int_{M} \phi d V_{g_{t}}=0$, as the constants lie in $\tilde{E}_{t}$.

As $\tilde{E}_{t}$ is just the constant functions in Section 2.1, we immediately deduce that Theorem 4.6 is true.

We will now construct the eigenvector $\beta_{t}$ of Proposition 4.7, using a sequence method. The proposition is stated again here.

Proof of Proposition 4.7. Let $y$ be the unique element of $C^{\infty}(M)$ satisfying $\int_{M} y d V_{g_{t}}=0$ and $a \Delta y=e$. To make $\beta_{t}=e+w$ and $a \Delta \beta_{t}=\lambda_{t} \beta_{t}$, we must find $w$ and $\lambda_{t}$ such that $a \Delta w=\lambda_{t}(e+w)-a \Delta e$. Define inductively a sequence of real numbers $\left\{\lambda_{i}\right\}_{i=0}^{\infty}$ and a sequence $\left\{w_{i}\right\}_{i=0}^{\infty}$ of elements of $C^{\infty}(M)$ beginning with $\lambda_{0}=w_{0}=0$. Let

$$
\lambda_{i}=2 \operatorname{vol}\left(M^{\prime}\right)\left(\int_{M} y\left(e+w_{i-1}\right) d V_{g_{t}}\right)^{-1},
$$

and let $w_{i}$ be the unique element of $C^{\infty}(M)$ satisfying $\int_{M} w_{i} d V_{g_{t}}=0$ and

$$
a \Delta w_{i}=\lambda_{i}\left(e+w_{i-1}\right)-a \Delta e
$$

Note that as $\int_{M} e d V_{g_{t}}=\int_{M} w_{i-1} d V_{g_{t}}=0$, the right-hand side has integral zero over $M$, and so $w_{i}$ exists. Thus, the sequences $\left\{\lambda_{i}\right\}_{i=0}^{\infty}$ and $\left\{w_{i}\right\}_{i=0}^{\infty}$ are well defined, provided only that the integral on the right-hand side of (A.34) is nonzero; we will prove later that the integral is bounded below by a positive constant.

If both sequences converge to $\lambda_{t}$ and $w$, respectively, say, then (A.35) implies that $a \Delta w=\lambda_{t}(e+w)-a \Delta e$ so that $\beta_{t}=e+w$ is an eigenvector of $a \Delta$ associated to $\lambda_{t}$. The rôle of (A.34) is as follows: multiply (A.35) by $y$ and integrate over $M$. Integrating by parts gives

$$
\int_{M} w_{i} a \Delta y d V_{g_{t}}=\lambda_{i} \int_{M} y\left(e+w_{i-1}\right) d V_{g_{t}}-\int_{M} e a \Delta y d V_{g_{t}}
$$

Substituting $e$ for $a \Delta y$ and recalling that $\int_{M} e^{2} d V_{g_{t}}=2 \operatorname{vol}\left(M^{\prime}\right)$, we get

$$
\int_{M} e w_{i} d V_{g_{t}}=\lambda_{i} \int_{M} y\left(e+w_{i-1}\right) d V_{g_{t}}-2 \operatorname{vol}\left(M^{\prime}\right)=0
$$


so that $\int_{M} e w_{i} d V_{g_{t}}=0$, and if $w$ is the limit of the sequence, then $\int_{M} e w d V_{g_{t}}=$ 0 .

Now as $\int_{M} w_{i} d V_{g_{t}}=\int_{M} e w_{i} d V_{g_{t}}=0, w_{i} \in\left(\tilde{E}_{t}\right)^{\perp}$ and Lemma A.18 gives

$$
a\left\|\nabla w_{i}\right\|_{L^{2}}^{2} \geq \gamma\left\|w_{i}\right\|_{L^{2}}^{2} .
$$

Multiplying (A.35) by $w_{i}$ and integrating over $M$ by parts gives

$$
\begin{aligned}
a\left\|\nabla w_{i}\right\|_{L^{2}}^{2} & =\int_{M} a\left|\nabla w_{i}\right|^{2} d V_{g_{t}} \\
& =\lambda_{i} \int_{M} w_{i}\left(e+w_{i-1}\right) d V_{g_{t}}-a \int_{M} \nabla w_{i} \cdot \nabla e d V_{g_{t}} \\
& \leq\left|\lambda_{i}\right|\left\|w_{i}\right\|_{L^{2}}\left(\|e\|_{L^{2}}+\left\|w_{i-1}\right\|_{L^{2}}\right)+a\left\|\nabla w_{i}\right\|_{L^{2}}\|\nabla e\|_{L^{2}} \\
& \leq\left|\lambda_{i}\right| a^{1 / 2} \gamma^{-1 / 2}\left\|\nabla w_{i}\right\|_{L^{2}}\left(\|e\|_{L^{2}}+\left\|w_{i-1}\right\|_{L^{2}}\right)+a\left\|\nabla w_{i}\right\|_{L^{2}}\|\nabla e\|_{L^{2}},
\end{aligned}
$$

applying (A.38) between the second and third lines, and Hölder inequality. Dividing by $a^{1 / 2} \gamma^{-1 / 2}\left\|\nabla w_{i}\right\|_{L^{2}}$ and using (A.38) on the left-hand side gives

$$
\gamma\left\|w_{i}\right\|_{L^{2}} \leq\left|\lambda_{i}\right|\left(\|e\|_{L^{2}}+\left\|w_{i-1}\right\|_{L^{2}}\right)+D_{0},
$$

where $D_{0}=(a \gamma)^{1 / 2}\|\nabla e\|_{L^{2}}$.

Define $D_{1}=\left(2 \operatorname{vol}\left(M^{\prime}\right)\right)^{-1} \int_{M} y e d V_{g_{t}}$. Then $y=D_{1} e+z$, where $\int_{M} z d V_{g_{t}}=$ $\int_{M} z e d V_{g_{t}}=0$. So $z \in\left(\tilde{E}_{t}\right)^{\perp}$, and by Lemma A.18,

$$
a\|\nabla z\|_{L^{2}}^{2} \geq \gamma\|z\|_{L^{2}}^{2} .
$$

As $\int_{M} z e d V_{g_{t}}=0$ and $e=a \Delta y=a D_{1} \Delta e+a \Delta z$, we have $\int_{M} z\left(D_{1} \Delta e+\Delta z\right) d V_{g_{t}}=$ 0 , so

$$
\|\nabla z\|_{L^{2}}^{2}=-D_{1} \int_{M} \nabla z \cdot \nabla e d V_{g_{t}} \leq D_{1}\|\nabla z\|_{L^{2}}\|\nabla e\|_{L^{2}}
$$

Multiplying by $(a \gamma)^{1 / 2}\|\nabla z\|_{L^{2}}^{-1}$ and substituting (A.41) into the left-hand side, then gives $\gamma\|z\|_{L^{2}} \leq D_{0} D_{1}$. Since $y=D_{1} e+z$ and $\int_{M} e w_{i-1} d V_{g_{t}}=0$ from (A.34), we find

$$
\begin{aligned}
\left|\lambda_{i}\right|^{-1} & =D_{1}+\frac{1}{2 \operatorname{vol}\left(M^{\prime}\right)} \int_{M} z w_{i-1} d V_{g_{t}} \\
& \geq D_{1}-\frac{\|z\|_{L^{2}}\left\|w_{i-1}\right\|_{L^{2}}}{2 \operatorname{vol}\left(M^{\prime}\right)} \\
& \geq D_{1}-\frac{D_{0} D_{1}\left\|w_{i-1}\right\|_{L^{2}}}{2 \operatorname{vol}\left(M^{\prime}\right) \gamma} .
\end{aligned}
$$

Now (A.40) and (A.43) are what we need to prove that the sequences $\left\{\lambda_{i}\right\}_{i=0}^{\infty}$ and $\left\{w_{i}\right\}_{i=0}^{\infty}$ are well defined and convergent, provided that $D_{0}$ is sufficiently small and $D_{1}$ sufficiently large. It can be shown that if $2 D_{0}^{2} \leq \gamma^{2} \operatorname{vol}\left(M^{\prime}\right)$ and 
$D_{1}$ is large enough, then the two sequences converge to $\lambda_{t}$ and $w$, respectively, satisfying

$$
\begin{gathered}
a \Delta w=\lambda_{t}(e+w)-a \Delta e, \quad \text { where }\|w\|_{L^{2}} \leq 2 D_{0} / \gamma \\
\left|\lambda_{t}\right|^{-1} \geq D_{1}-\frac{D_{0}^{2} D_{1}}{\gamma^{2} \operatorname{vol}\left(M^{\prime}\right)} \geq \frac{1}{2} D_{1}, \quad \text { so that }\left|\lambda_{t}\right| \leq 2 D_{1}^{-1} .
\end{gathered}
$$

The proof uses the same sort of reasoning as Lemma 3.2, and will be left to the reader.

Now look at $D_{0}$ and $D_{1}$ more closely. Firstly, $D_{0}=(a \gamma)^{1 / 2}\|\nabla e\|_{L^{2}}$, and $e$ is defined by $\sigma^{\prime}$ and $\sigma^{\prime \prime}$ chosen just before Lemma A.17. In fact, $e=c^{\prime} \sigma^{\prime}-c^{\prime \prime} \sigma^{\prime \prime}$, where $c^{\prime}$ and $c^{\prime \prime}$ are close to 1 , as $\operatorname{vol}\left(M^{\prime}\right)=\operatorname{vol}\left(M^{\prime \prime}\right)$. But $\sigma^{\prime}, \sigma^{\prime \prime}$ are defined in the same way as $\beta_{1}, \beta_{2}$ of Section 2.1, and an estimate analogous to (2.9) of Section 2.4 applies to them, from which we find that $D_{0}=O\left(t^{(n-2)^{2} /(n+1)}\right)$ for small $t$. Because $\|w\|_{L^{2}} \leq 2 D_{0} / \gamma$, this gives that $\|w\|_{L^{2}}=O\left(t^{(n-2)^{2} /(n+1)}\right)$.

Secondly, we need to estimate $D_{1}$. Let $\xi^{\prime}$ be the Green's function of $a \Delta$ at $m^{\prime}$ on $M^{\prime}$ satisfying $a \Delta \xi^{\prime}=\delta_{m^{\prime}}-1 / \operatorname{vol}\left(M^{\prime}\right)$ in the sense of distributions. Then $\xi^{\prime}$ has a pole of the form $D_{2}|v|^{2-n}+O^{\prime \prime}\left(|v|^{1-n}\right)$ at $m^{\prime}$, where $D_{2}=(n-$ $2)^{-1} \omega_{n-1}^{-1}$. Similarly, we may define the Green's function $\xi^{\prime \prime}$ of $a \Delta$ at $m^{\prime \prime}$ on $M^{\prime \prime}$.

The application of this is in modelling the function $y$ on $M$. We may view $M$, approximately, as being made up of the unions of $M^{\prime}$ and $M^{\prime \prime}$, each with a small ball of radius $t$ cut out. To get a function $\xi$ on $M$ with $\Delta \xi$ close to $\operatorname{vol}\left(M^{\prime}\right)^{-1}$ on the part coming from $M^{\prime}$, and close to $-\operatorname{vol}\left(M^{\prime \prime}\right)^{-1}$ on the part coming from $M^{\prime \prime}$, we try $\xi$ equal approximately to $d^{\prime}-\xi^{\prime}$ on $M^{\prime}$ and $\xi^{\prime \prime}-d^{\prime \prime}$ on the part coming from $M^{\prime \prime}$, for constants $d^{\prime}$ and $d^{\prime \prime}$. To join these two functions together on the neck, we must have $d^{\prime}+d^{\prime \prime}=2 D_{2} t^{2-n}+O\left(t^{1-n}\right)$, and for $\int_{M} \xi d V_{g_{t}}=0$, we must have $d^{\prime} \operatorname{vol}\left(M^{\prime}\right)=d^{\prime \prime} \operatorname{vol}\left(M^{\prime \prime}\right)+O\left(t^{1-n}\right)$. As $\operatorname{vol}\left(M^{\prime}\right)=\operatorname{vol}\left(M^{\prime \prime}\right)$, this gives $d^{\prime}=D_{2} t^{2-n}+O\left(t^{1-n}\right)=d^{\prime \prime}$.

But $e$ is approximately equal to 1 on $M^{\prime}$ and to -1 on $M^{\prime \prime}$ so that $e \sim$ $\operatorname{vol}\left(M^{\prime}\right) a \Delta \xi$. Therefore, $y \sim \operatorname{vol}\left(M^{\prime}\right) \xi$, and $D_{1}=\left(2 \operatorname{vol}\left(M^{\prime}\right)\right)^{-1} \int_{M} y e d V_{g_{t}} \sim$ $d^{\prime} \operatorname{vol}\left(M^{\prime}\right)$. So finally, we conclude that $D_{1}=D_{2} \operatorname{vol}\left(M^{\prime}\right) t^{2-n}+O\left(t^{1-n}\right)$. This validates the claim that $D_{1}$ is large for small $t$, that was used earlier to ensure convergence.

Taking the limit over $i$ in (A.34), we find that

$$
\lambda_{t}^{-1}=D_{1}+\frac{1}{2 \operatorname{vol}\left(M^{\prime}\right)} \int_{M} z w d V_{g_{t}}
$$

so using estimates on $D_{1}, z$, and $w$ gives that $\lambda_{t}=O\left(t^{n-2}\right)$ for small $t$, one of the conclusions of the proposition. Also, if $e$ is a first approximation to $\beta_{t}$, then $\lambda_{t} y$ is the second, and the model of $y$ above gives a model of $\beta_{t}$. So we see that (4.11) holds for $\beta_{t}$, completing the proof. 
Finally, we show that Lemma A.18 may be modified further to apply to functions orthogonal to both 1 and the eigenvector $\beta_{t}$ constructed in Proposition 4.7 .

LEMMA A.19. Let $\left\{g_{t}: t \in(0, \delta)\right\}$ be the family of metrics defined on $M=$ $M^{\prime} \# M^{\prime \prime}$ in Section 4.2. Then for small $t$, if $\phi \in L_{1}^{2}(M)$ satisfies $\langle\phi, 1\rangle=\left\langle\phi, \beta_{t}\right\rangle=$ 0 in either the $L^{2}$ or the $L_{1}^{2}$ inner product, then

$$
\int_{M} a|\nabla \phi|^{2} d V_{g_{t}} \geq \gamma \int_{M} \phi^{2} d V_{g_{t}}
$$

Here $\beta_{t}$ is the eigenvector of a constructed in Proposition 4.7.

Proof. The proof is almost the same as that of Lemma A.18. Note that as $1, \beta_{t}$ are eigenvectors of $\Delta$, orthogonality to them with respect to the $L^{2}$ and $L_{1}^{2}$ inner products is equivalent, and so we may suppose that $\langle$,$\rangle is the inner$ product of $L^{2}(M)$. Let $\xi$ be the unique element of $\tilde{E}_{t}$ satisfying $\left\langle\xi, \beta_{t}\right\rangle=1$ and $\int_{M} \xi d V_{g_{t}}=0$. If $\phi \in L_{1}^{2}(M)$ satisfies $\langle\phi, 1\rangle=\left\langle\phi, \beta_{t}\right\rangle=0$, then $\phi-\langle\phi, \xi\rangle \beta_{t} \in \tilde{E}_{t}^{\perp}$, taken with respect to the $L^{2}$ norm. So by Lemma A.18,

$$
\int_{M} a\left|\nabla \phi-\langle\phi, \xi\rangle \nabla \beta_{t}\right|^{2} d V_{g_{t}} \geq \gamma \int_{M}\left(\phi-\langle\phi, \xi\rangle \beta_{t}\right)^{2} d V_{g_{t}}
$$

But as $\beta_{t}$ is an eigenvalue of $\Delta$, it is orthogonal to $\phi$ in both $L^{2}$ and $L_{1}^{2}$ norms, so this equation becomes

$$
\int_{M} a\left(|\nabla \phi|^{2}+\langle\phi, \xi\rangle^{2}\left|\nabla \beta_{t}\right|\right) d V_{g_{t}} \geq \gamma \int_{M}\left(\phi^{2}+\langle\phi, \xi\rangle^{2} \beta_{t}^{2}\right) d V_{g_{t}} .
$$

Now $\lambda_{t}<\gamma$ for small $t$ so that $\int_{M} a\left|\nabla \beta_{t}\right|^{2} d V_{g_{t}} \leq \gamma \int_{M} \beta_{t}^{2} d V_{g_{t}}$, and subtracting this multiplied by $\langle\phi, \xi\rangle^{2}$ from (A.48) gives (A.46).

Thus, Theorem 4.8 is true.

ACKNOWLEdgments. The author would like to thank Simon Donaldson for many useful conversations and Rafe Mazzeo and the referees for helpful comments.

\section{REFERENCES}

[1] T. Aubin, Nonlinear Analysis on Manifolds. Monge-Ampère Equations, Grundlehren der Mathematischen Wissenschaften, vol. 252, SpringerVerlag, New York, 1982.

[2] R. Bartnik, The mass of an asymptotically flat manifold, Comm. Pure Appl. Math. 39 (1986), no. 5, 661-693.

[3] M. Gromov and H. B. Lawson Jr., The classification of simply connected manifolds of positive scalar curvature, Ann. of Math. (2) 111 (1980), no. 3, 423-434.

[4] L. Hörmander, Linear Partial Differential Operators, Die Grundlehren der mathematischen Wissenschaften, vol. 116, Springer-Verlag, Berlin, 1963.

[5] D. D. Joyce, Hypercomplex and quaternionic manifolds, and scalar curvature on connected sums, Ph.D. thesis, University of Oxford, Oxford, 1992. 
[6] O. Kobayashi, Scalar curvature of a metric with unit volume, Math. Ann. 279 (1987), no. 2, 253-265.

[7] J. M. Lee and T. H. Parker, The Yamabe problem, Bull. Amer. Math. Soc. (N.S.) 17 (1987), no. 1, 37-91.

[8] R. Mazzeo, D. Pollack, and K. Uhlenbeck, Connected sum constructions for constant scalar curvature metrics, Topol. Methods Nonlinear Anal. 6 (1995), no. 2, 207-233.

[9] R. M. Schoen, Conformal deformation of a Riemannian metric to constant scalar curvature, J. Differential Geom. 20 (1984), no. 2, 479-495.

[10] - Variational theory for the total scalar curvature functional for Riemannian metrics and related topics, Topics in Calculus of Variations (Montecatini Terme, 1987) (M. Giaquinta, ed.), Lecture Notes in Mathematics, vol. 1365, Springer, Berlin, 1989, pp. 120-154.

[11] R. M. Schoen and S.-T. Yau, On the structure of manifolds with positive scalar curvature, Manuscripta Math. 28 (1979), no. 1-3, 159-183.

[12] N. S. Trudinger, Remarks concerning the conformal deformation of Riemannian structures on compact manifolds, Ann. Scuola Norm. Sup. Pisa (3) 22 (1968), 265-274.

[13] E. Witten, A new proof of the positive energy theorem, Comm. Math. Phys. 80 (1981), no. 3, 381-402.

[14] H. Yamabe, On a deformation of Riemannian structures on compact manifolds, Osaka Math. J. 12 (1960), 21-37.

Dominic Joyce: Lincoln College, Oxford, OX1 3DR, UK

E-mail address: dominic. joyce@1 incoln.ox.ac.uk 


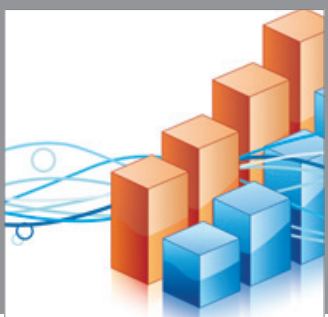

Advances in

Operations Research



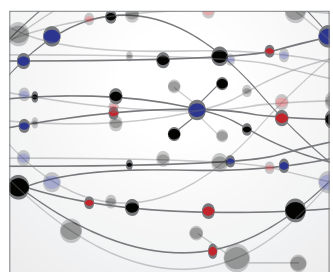

\section{The Scientific} World Journal


International Journal of

Mathematics and

Mathematical

Sciences
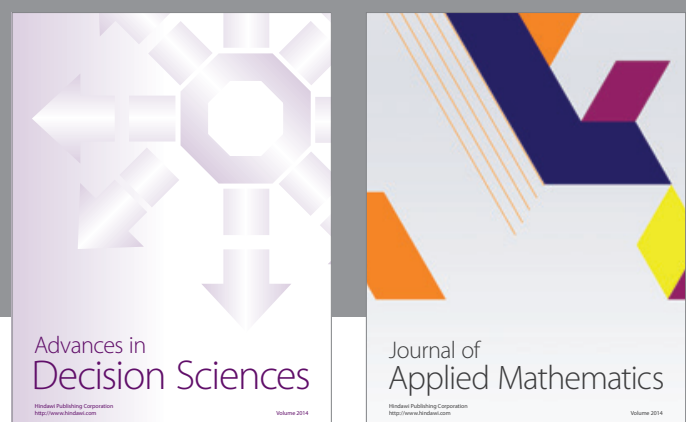

Journal of

Applied Mathematics
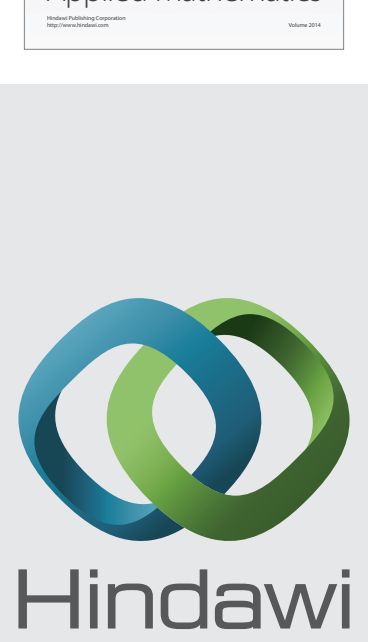

Submit your manuscripts at http://www.hindawi.com
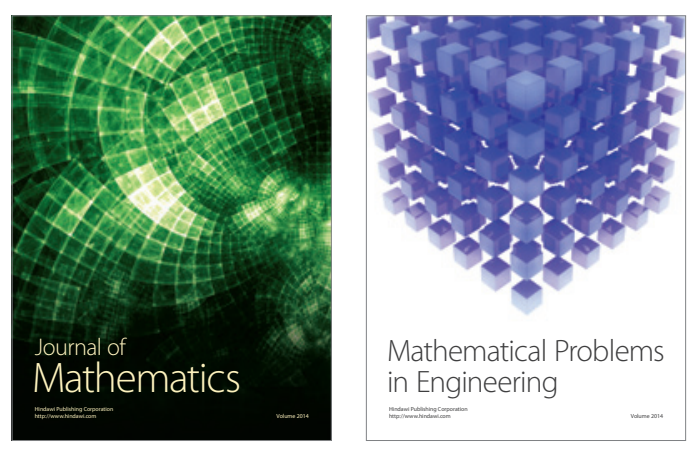

Mathematical Problems in Engineering
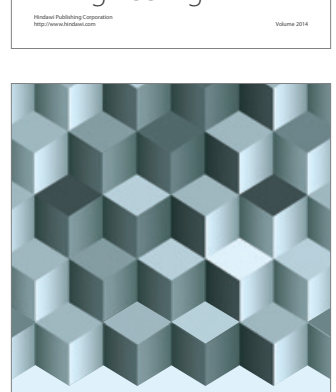

Journal of

Function Spaces
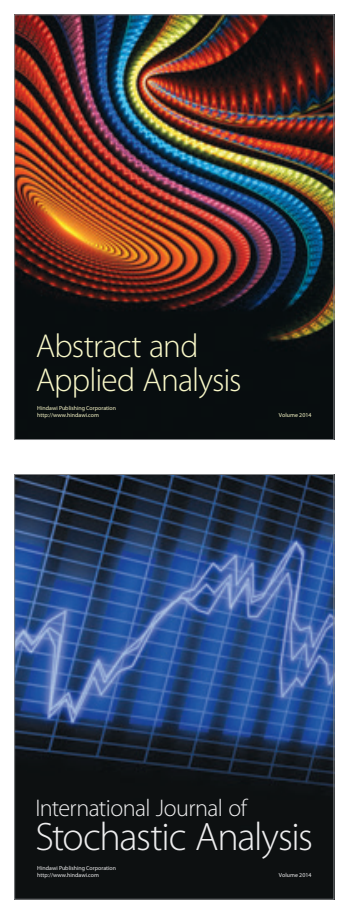

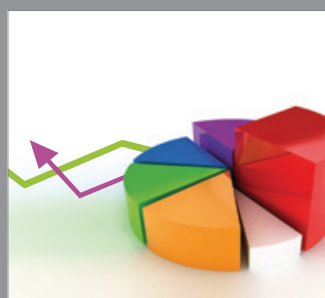

ournal of

Probability and Statistics

Promensencen
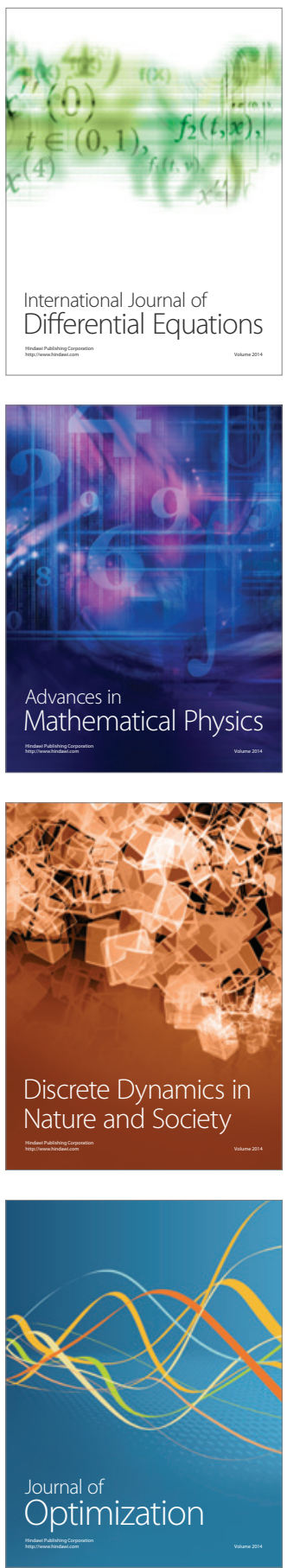